\title{
Total Synthesis of Dimeric HPI Alkaloids
}

\author{
Xianfu Shen $\cdot$ Yongyun Zhou $\cdot$ Yongkai Xi $\cdot$ \\ Jingfeng Zhao $\cdot$ Hongbin Zhang
}

Received: 13 January 2016/Accepted: 19 February 2016/Published online: 11 March 2016

(C) The Author(s) 2016. This article is published with open access at Springerlink.com

\begin{abstract}
In this paper, we report a full account of the synthesis of dimeric hexahydropyrroloindole alkaloids and its analogues. The key feature of our new strategy is the novel catalytic copper $(10 \%)$ mediated intramolecular arylations of $o$-haloanilides followed by intermolecular oxidative dimerization of the resulting oxindoles in one pot. This sequential reaction leads to the key intermediates for the synthesis of (+)-chimonanthine, (+)-folicanthine, (-)-calycanthine and (-)-ditryptophenaline.

Graphical Abstract In the presence of catalytic amount of cuprous iodide (10\%), an intramolecular arylation of $o$ haloanilides followed by an intermolecular oxidative dimerization of the resulting oxindoles leads to a common intermediate for the synthesis of $(+)$-chimonanthine, $(+)$-folicanthine and (-)-calycanthine. Based on this cascade sequence, we also developed a flexible strategy towards the asymmetric syntheses of dimeric HPI alkaloids (-)-ditryptophenaline and its analogues.
\end{abstract}

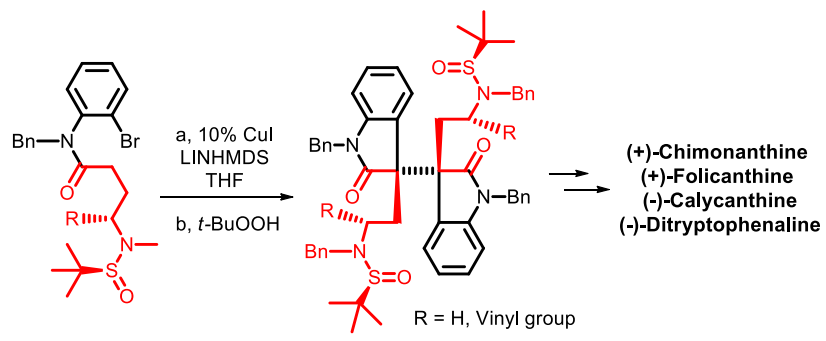

Keywords Copper catalyzed reaction · Oxidative dimerization · Total synthesis · Hexahydropyrroloindole ·

Alkaloids

Xianfu Shen and Yongyun Zhou have contributed equally to this paper.

Electronic supplementary material The online version of this article (doi:10.1007/s13659-016-0092-8) contains supplementary material, which is available to authorized users.

X. Shen $\cdot$ Y. Zhou $\cdot$ Y. Xi · J. Zhao $\cdot$ H. Zhang $(\bowtie)$

Key Laboratory of Medicinal Chemistry for Natural Resource (Yunnan University), Ministry of Education, School of Chemical Science and Technology, Yunnan University, Kunming 650091, People's Republic of China

e-mail: zhanghb@ynu.edu.cn

\section{Introduction}

The hexahydropyrroloindole (HPI) structure-unit presents in a large class of natural products isolated from plants, microorganisms and fungi (for selected reviews and book chapters, see: [1-8]). Representative natural product of this family is physostigmine (Fig. 1, 1) which was isolated from the seeds of the calabar bean plant and is currently used to treat myasthenia gravis, glaucoma, Alzheimers disease, delayed gastric emptying and orthostatic hypotension [5, 7]. There are a number of alkaloids containing more than one HPI unit and 


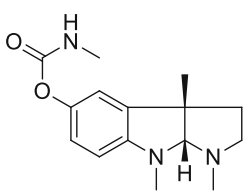

1: Physostigmine

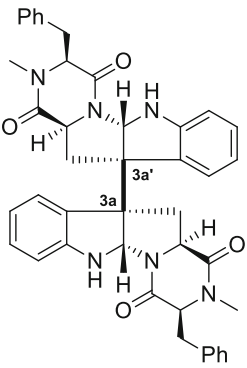

5: (-)-Ditryptophenaline

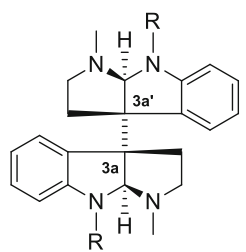

2: $\mathrm{R}=\mathrm{H},(+)$-Chimonanthine 3: $\mathrm{R}=\mathrm{Me},(+)$-Folicanthine

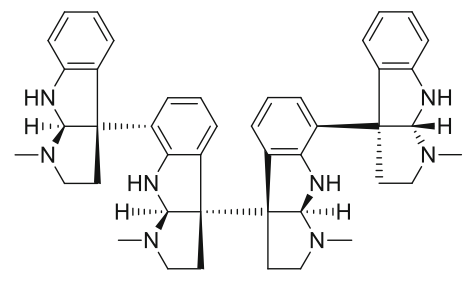

6: Quadrigemine C

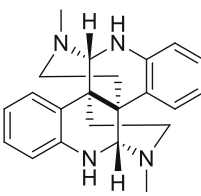

4: (-)-Calycanthine
Fig. 1 Natural HPI alkaloids

some of them contain a unique vicinal $\mathrm{C} 3 \mathrm{a}-\mathrm{C} 3 \mathrm{a}^{\prime}$ quaternary carbon center [1, 4]. The stereocontrol synthesis of the congested all-carbon quaternary stereocenters in these alkaloids presents a formidable challenge [9-16]. In 1999, Overman and his team completed the first enantioselective synthesis of dimeric alkaloid (+)-calycanthine (Fig. 1, 4) and (-)-chimonanthine (Fig. 1, 2) [17]. Utilization of the same strategy, they also successfully synthesized a number of other dimeric and oligomeric HPI alkaloids [17-27]. In 2007, Mavassaghi reported a reductive radical dimerization strategy for the syntheses of dimeric HPI alkaloids (Fig. 1) [28, 29]. Based on this reductive dimerization reaction, a number of important works have been published towards the syntheses of dimeric HPI alkaloids (for selected syntheses of dimeric HPI alkaloids employed Movassaghi's reductive dimerization, see: [3037]). Prompted by the success of reductive dimerization strategies, studies on the oxidative dimerization of tryptamine and tryptophan derivatives have been revived and syntheses of natural HPI alkaloids, especially chimonanthine, folicanthine and ditryptophenaline, have been achieved by a number of research groups (oxidative dimerization of tryptamine and tryptophan derivatives as the key steps for the syntheses of dimeric HPI alkaloids before 2007: [38-58]).

Careful examination of literature related to the total syntheses of dimeric HPI alkaloids, we found that tryptamine or tryptophan derivatives were used frequently as starting materials [38-58], few examples had been documented by application of non-indole and/or non-oxindole starting materials [59]. To some extent, utilization of tryptamine and tryptophan derivatives as starting materials might limited the access of structurally diverse dimeric analogues. Therefore, it is of importance to develop alternative approaches towards the synthesis of the target dimeric HPI natural molecules as well as its analogues for the interests of medicinal chemistry.
A major focus of our research group is the use of metal mediated sequential reactions to assemble the key structure units of the target molecules [60-63]. Our synthesis of the HPI alkaloids initiated in the early 2008. We successfully developed a sequential reaction for the synthesis of mesembrine [60] and esermethole [61] using palladium chemistry. In 2012, we developed a copper catalyzed arylation of $o$-bromoanilides assisted by a remote sulfinylamide or carbamate auxiliary [62]. Very recently, we established a novel copper catalyzed asymmetric arylationoxidative dimerization of $o$-haloanilide derivatives (Scheme 1) to construct the vicinal $\mathrm{C} 3 \mathrm{a}-\mathrm{C} 3 \mathrm{a}^{\prime}$ all carbon quaternary stereocenters required for the synthesis of dimeric HPI compounds [64]. In this paper, we report our full accounts of new strategy towards the synthesis of (+)chimonanthine, (+)-folicanthine, (-)-calycanthine, (-)ditryptophenaline and its analogues.

\section{Results and Discussion}

The syntheses of dimeric HPI alkaloids began in the early 1960, and after endeavours of many research groups, the bio-inspired oxidative dimerization reaction of tryptamine and tryptophan derivatives has been developed to be a powerful method for the construction of dimeric HPI alkaloids [38-58, 64]. In comparison with oxidative dimerization of tryptamine and tryptophan derivatives, however, few oxidative dimerizations of oxindole derivatives have been reported [65-74]. To the best of our knowledge, only five papers reported direct oxidative dimerization of oxindoles derivatives. Except for the method established by Rodrigo which provided the dimeric intermediate in $61 \%$ overall yield and good diastereoselectivity ( $d l$ :meso isomers $=53: 8$, Scheme 2) [67], other methods unfortunately suffered from low yields [65] or
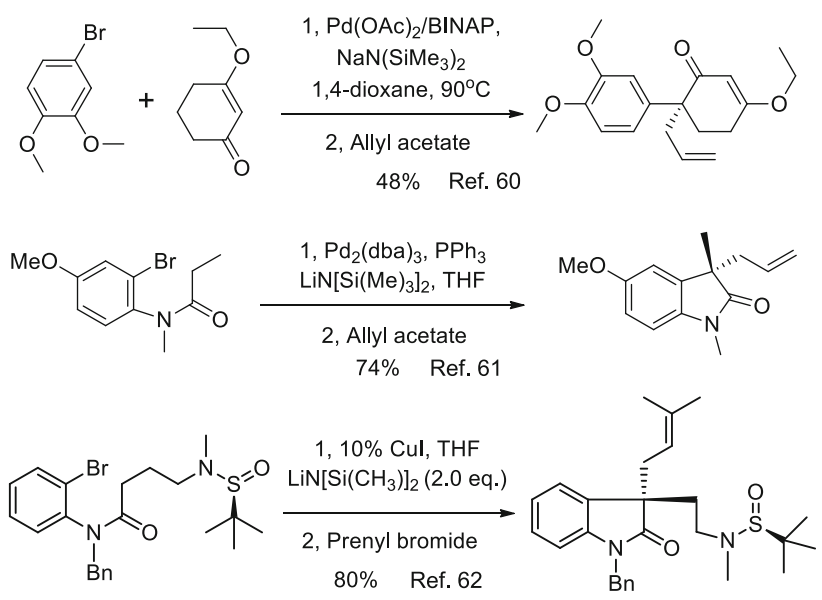

Scheme 1 Sequential process for natural product synthesis 

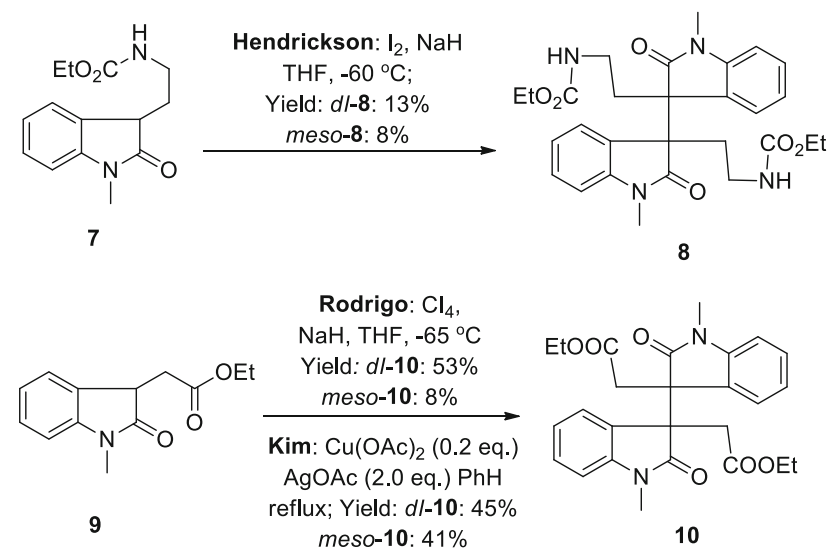

Scheme 2 Oxidative dimerization of oxindoles in the literature

poor diastereoselectivity $[66,68,69]$. Before we conducted this research, no asymmetric oxidative dimerization of oxindoles had been developed to form the vicinal C3-C ${ }^{\prime}$ all-carbon quaternary centers.

Recently, we disclosed an asymmetric synthesis of debromoflustramine and its analogues through a copper mediated arylation followed by oxindole-alkylation [62]. We envisioned that this copper catalysed cyclization process (Scheme 1) might be applied to the synthesis of dimeric HPI alkaloids indicated in Fig. 1. A retrosynthetic analysis is outlined in Scheme 3. Key to our new strategy is to develop a sequential reaction that combines metal catalysed arylation with an in situ oxidative dimerization of the resulting oxindole intermediates (Scheme 3, converting 12 to 13). Based on this new sequential process, we would be able to synthesize the dimeric HPI alkaloids starting from $o$-haloanilide 12. The amides (12) bearing a chiral sulfinyl amide unit $[(S)$ or $(R)$-tert-butanesulfinamide] could be synthesized according to our previous procedure. We were curious to know whether the key intermediates (13, Scheme 3), containing the vicinal C3-C3' all-carbon quaternary centers required for dimeric HPI alkaloids, could be formed diastereoselectively in a one-pot manner by a copper catalyzed arylation of $o$-haloanilide (12) followed by an oxidative dimerization of the newly generated oxindole intermediates in the presence of a suitable oxidant.

Our synthesis started from commercially available $o$ bromoaniline and $\gamma$-butyrolactone, and $(S)$-(-)-tert-butanesulfin-amide was used to introduce the nitrogen atom and also served as a chiral auxiliary. Amide 12a was synthesized in $68 \%$ overall yield in six steps according to our previous procedure [62] (Scheme 4). Amide 12b was also prepared from 17a in a $97 \%$ yield. With amides (12a and 12b) in hand, we next began to explore the key copper mediated sequential arylation-dimerization of $o$-bromoanilides, in the hope that the oxidative dimerization might also be effected by copper salts in an efficient and economic "one pot" operation.

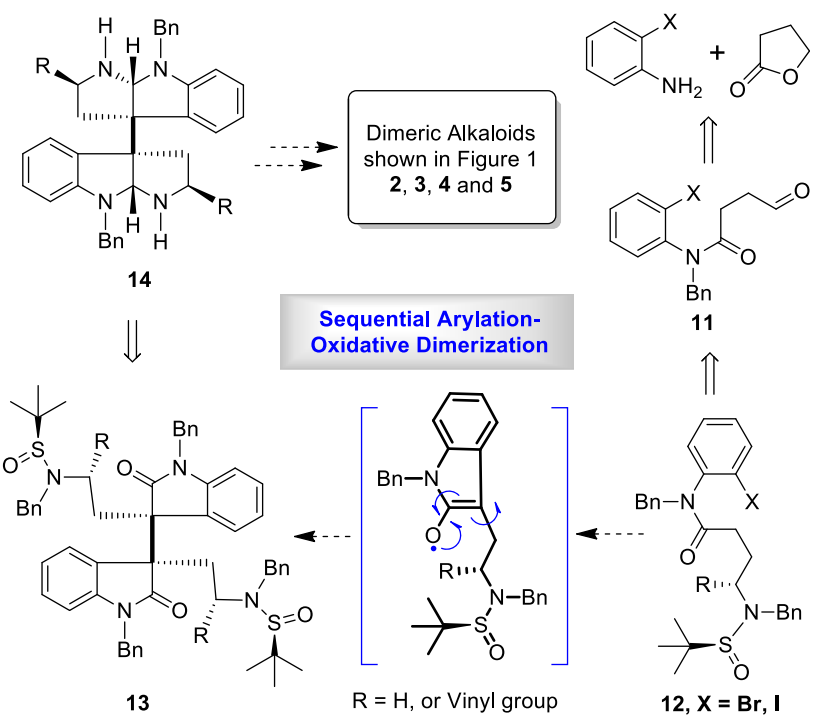

Scheme 3 Synthetic analysis of dimeric HPI alkaloids

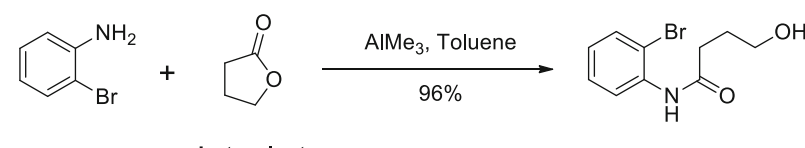

o-bromoaniline $\gamma$-butyrolactone

$$
96 \% \mid \begin{gathered}
\mathrm{CH}_{3} \mathrm{CN}-\mathrm{DMF} \\
\mathrm{BnCl}, \mathrm{Cs}_{2} \mathrm{CO}_{3}
\end{gathered}
$$

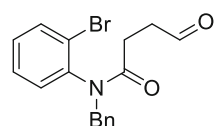

Bn

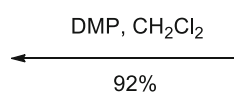

$94 \% \mid \begin{array}{r}\text { 11a } \\ \mathrm{Ti}(\mathrm{OEt})_{4}, \mathrm{THF}, 60^{\circ} \mathrm{C}\end{array}$

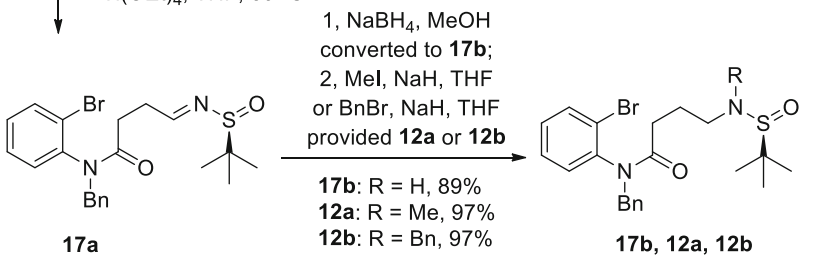

Scheme 4 Syntheses of amides 12a and 12b

The initial experiment was conducted with amide 12b under our previous optimized reaction condition [62], namely $\mathrm{LiN}\left(\mathrm{SiMe}_{3}\right)_{2}$ and $\mathrm{CuI}$ in THF at reflux (Table 1, entry 1). This condition unfortunately gave a complex mixture, with the isolated product being $N$-benzyl-o-bromoaniline (18, $16 \%$ yield). After several unsuccessful experiments, we finally found that the desired sequential reaction could be realized smoothly in toluene (Table 1, entry 6). Arylation of sulfinamide 12b with catalytic amount of cuprous iodide (10\% eq.) and lithium bis(trimethylsilyl)amide (2.0 eq.) in toluene, followed by oxidation with anhydrous tert-butylhydroperoxide resulted 
Table 1 Attempted conditions for copper catalyzed sequential arylation-oxidative dimerization of $o$-bromoanilide 12a and 12b

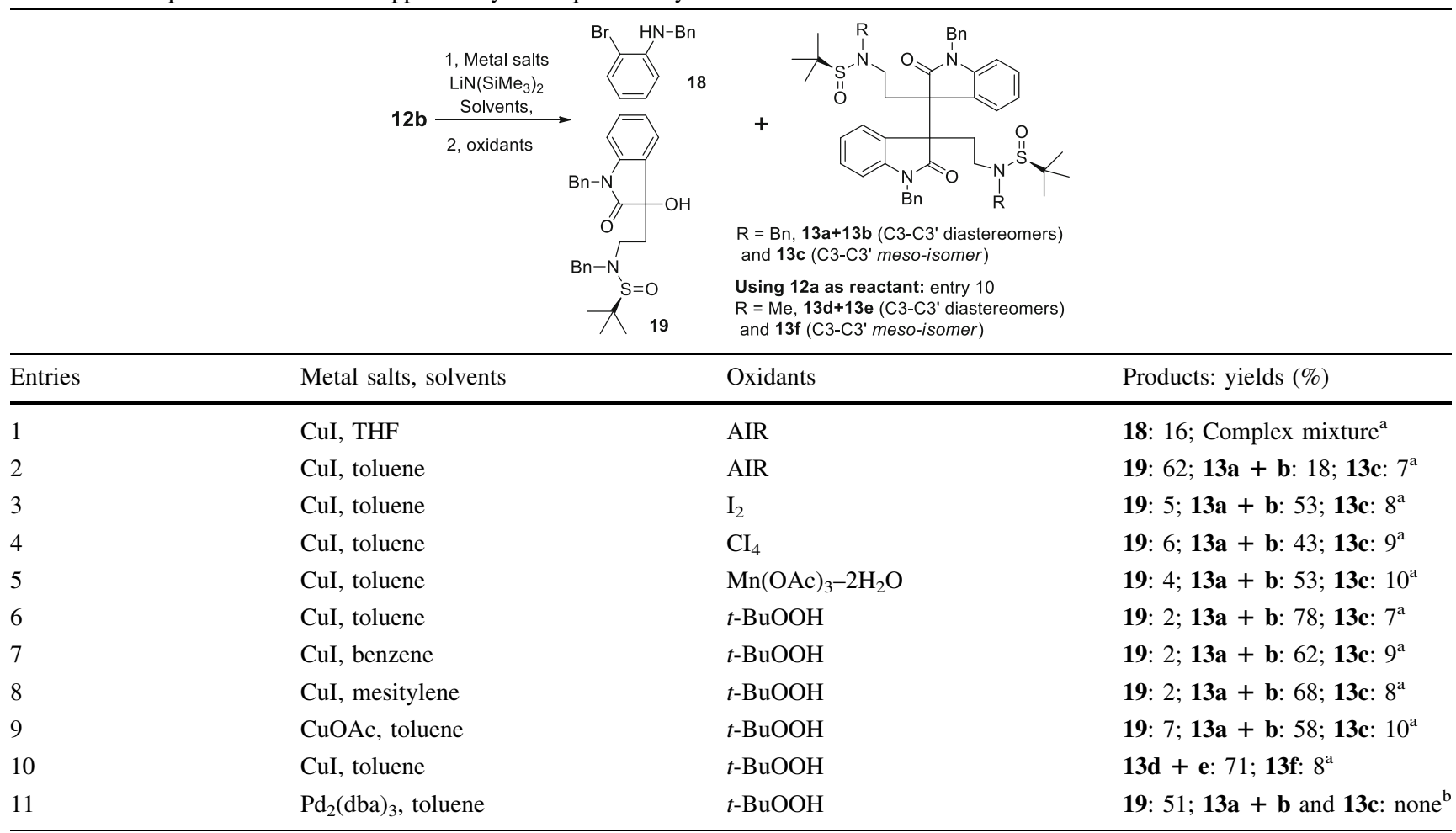

Yields represent isolated yields, average of two runs. All arylation reactions were conducted at 2 mmol scale under argon for 5-6 h, then addition of oxidants $(2.2 \mathrm{mmol})$ or open to air

${ }^{\text {a }}$ Copper salts (0.1 eq.), $\mathrm{LiN}\left(\mathrm{SiMe}_{3}\right)_{2}\left(2.0\right.$ eq.), designated solvent, $80{ }^{\circ} \mathrm{C}$

b $\mathrm{Pd}_{2}(\mathrm{dba})_{3}$ (0.05 eq.), $\mathrm{Ph}_{3} \mathrm{P}(0.2$ eq. $), \mathrm{LiN}\left(\mathrm{SiMe}_{3}\right)_{2}$ (2.0 eq.), toluene, $80{ }^{\circ} \mathrm{C}$

in dimeric diastereomers $(\mathbf{1 3} \mathbf{a}+\mathbf{1 3} \mathbf{b}$, as a 5:1 mixture of diastereoisomers in $78 \%$ yield, and 13c: the meso isomer in $7 \%$ yield). We also isolated small amount of the C3hydroxy-oxindole (19). Although a number of oxidants could be used to generate the desired dimeric product (13a $+\mathbf{1 3 b}$ ), anhydrous tert-butyl hydroperoxide proved to be the best additive for this sequential arylation-oxidative dimerization. Next we carried out the sequential procedure with amide 12a under the optimized reaction condition. The dimeric diastereomers $(\mathbf{1 3 d}+\mathbf{1 3 e}$, as a mixture of diastereoisomers) were obtained in $71 \%$ yield, we also isolated the meso isomer $\mathbf{1 3 f}$ in $8 \%$ yield (Table 1, entry $10)$.

In order to know whether the oxidative dimerization was promoted solely by tert-butyl hydroperoxide, we conducted an arylation with tris(dibenzylideneacetone)dipalladium $\left[\mathrm{Pd}_{2}(\mathrm{dba})_{3}\right]$ [61] followed by oxidation with $t$-BuOOH. It was noteworthy that only $\mathrm{C} 3$-hydroxy-2-oxindole product (19, Table 1 , entry 11 , as a mixture of diastereomers at C3 position, Scheme 5) was obtained. We next carried out arylation with $\mathrm{Pd}_{2}(\mathrm{dba})_{3}$ without addition of $t-\mathrm{BuOOH}$ and successfully obtained oxindole 19a in $61 \%$ yield. The oxindole (19a) was then subjected to oxidation in the presence of $\mathrm{LiN}\left(\mathrm{SiMe}_{3}\right)_{2}$ and $t$ - $\mathrm{BuOOH}$ (see Scheme 5) and provided C3-hydroxy-2-oxindole (19) as the sole product.

The fact that no dimerization products $(\mathbf{1 3 a}, \mathbf{1 3 b}$ or $\mathbf{1 3 c})$ formed in the absence of a copper salt suggests that the copper(II) ion, rather than tert-butyl hydroperoxide, plays the role of oxidizing the carbanion to a radical in the dimerization process. Tert-butyl hydroperoxide just serves as an oxidant to convert copper(I) to copper(II) in the oxidative dimerization process.

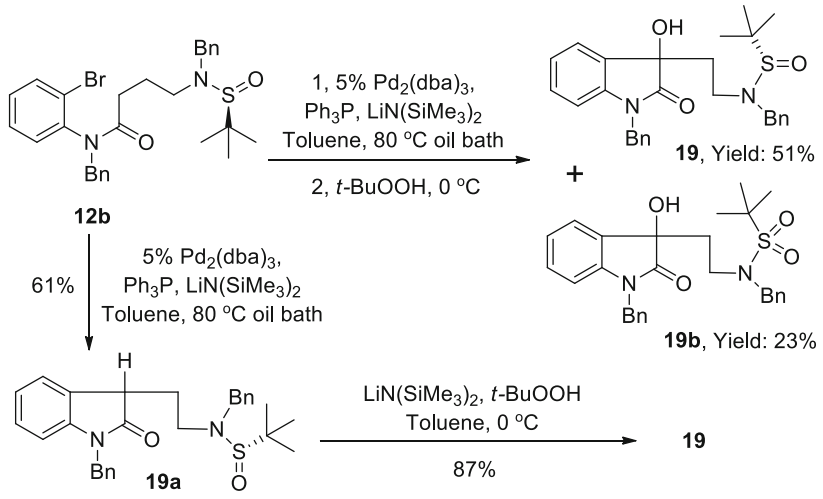

Scheme 5 Palladium catalyzed arylation of $o$-bromoanilide $\mathbf{1 2 b}$ 
Next, we came to the issue of determining diastereoselectivity induced by remote tert-butanesulfinamide moiety. Although the major product (13a and 13b) of this reaction was an unseparatable mixture of diastereoisomers (C $3 S-$ $\mathrm{C} 3^{\prime} S$ and $\mathrm{C} 3 R-\mathrm{C}^{\prime} R$ ) by silica gel column chromatography, the ratio could be readily determined by proton nuclear magnetic resonance $\left({ }^{1} \mathrm{H}-\mathrm{NMR}\right.$; $84: 16$, see Electronic supplementary material). The enantioselectivity for the formation of vicinal $\mathrm{C} 3-\mathrm{C} 3{ }^{\prime}$ quaternary carbon center was also determined after oxidation of the tert-butylsulfinyl group with 3-chloroperbenzoic acid ( $m$-CPBA) in dichloromethane. A $66 \%$ enantioselective excess was recorded (see Electronic supplementary material for chiral HPLC analysis) under our optimum reaction condition (Table 1, entry 6). The enantioselectivity for compounds 13d and 13e was also determined after oxidation of the tertbutylsulfinyl group with 3-chloroperbenzoic acid ( $m$-CPBA) and relatively low enantioselective excess (35\%) was observed. To the best of our knowledge, this is the first example of copper catalyzed sequential arylation-dimerization of an $o$-bromoanilide, a high-yield procedure and also the first asymmetric oxidative dimerization of an oxindole derivative with good diastereoselectivity $(d r$ $>10: 1)$ and enantioselectivity $(e e=66 \%)$. The absolute stereochemistry was late confirmed by the total synthesis of (+)-chimonanthine (Scheme 10). A working hypothesis was proposed for prediction of $\mathrm{C} 3-\mathrm{C}^{\prime}$ configuration in Scheme $6 . \pi-\pi$ Stack (for a review for $\pi-\pi$.stack in asymmetric synthesis, see: $[75,76])$ might play important role for the enantioselectivity as well as diastereoselectivity.

In order to access the starting material for the synthesis of ditryptophenaline, we started the synthesis of amide 12c (Scheme 7). Nucleophilic addition of vinyl magnesium bromide to aldimine 17a unfortunately provided two isolatable diastereisomers $(\mathbf{2 0 a}$ and $\mathbf{2 0 b})$ in a ratio of 3.4-1 (see Scheme 7). This problem was soon fixed by addition of Grignard reagent to aldimine $\mathbf{1 7 c}$, a surrogate prepared from $o$-iodoaniline. Better diastereoselectivity $(d r=10: 1$, see Scheme 7) was obtained by 1,2-addition of vinyl magnesium bromide to $o$-iodoanilide 17c. After benzylation, we obtained 12c in $58 \%$ yield in six steps from iodoaniline. The absolute configuration for the newly generated chiral center of compound 12c was deduced by Cram's chelation model [77] and late confirmed by X-ray crystallography (Schemes 8,9) and our total synthesis of (-)-ditryptophenaline (Scheme 11).

We next conducted the arylation-oxidative dimerization reaction with substrate 12c under the optimized condition. This reaction afforded the dimerization products in good yield, and all dimeric diastereomers were isolated by silica gel column chromatography, with the desired isomer (21a)
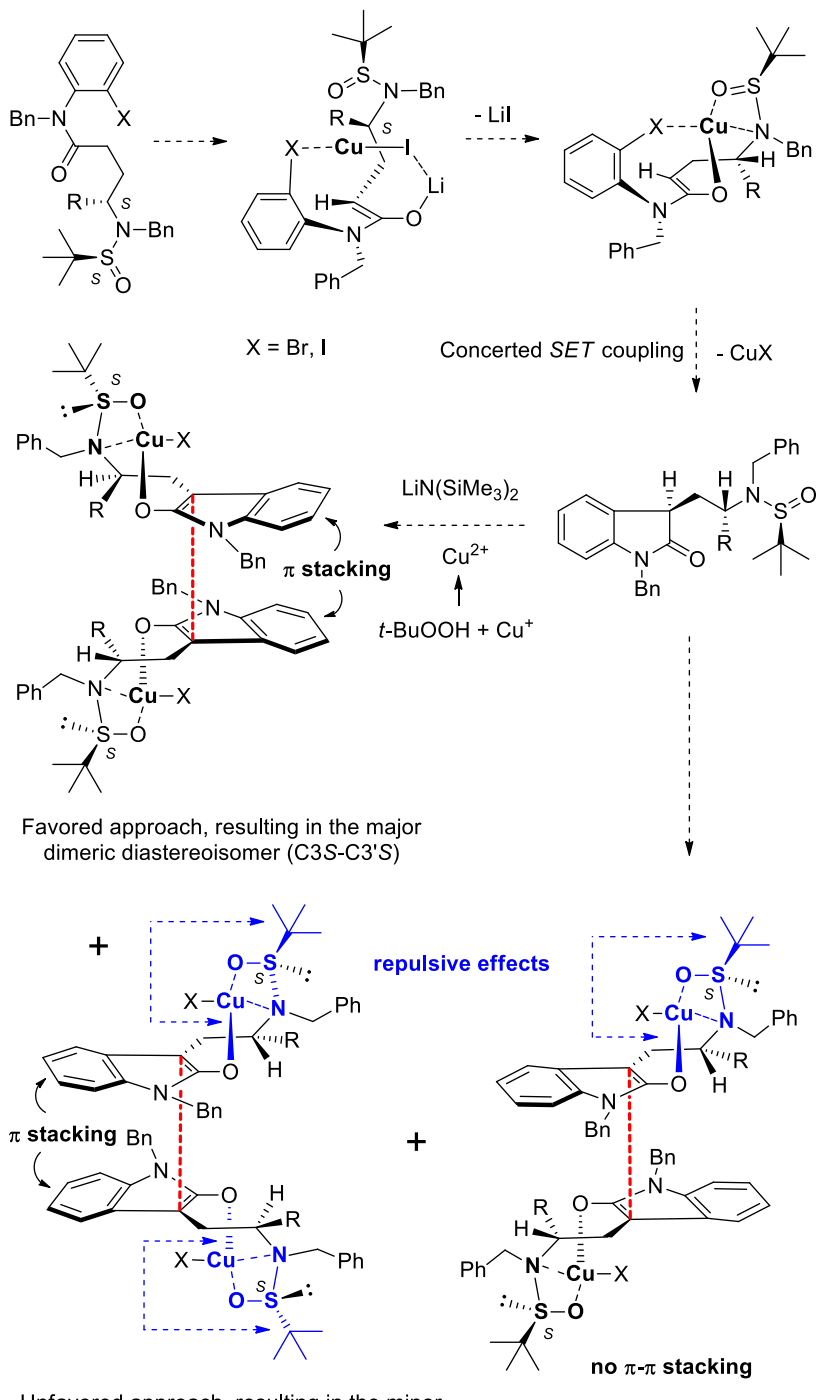

Unfavored approach, resulting in the minor dimeric diastereoisomer $\left(\mathrm{C} 3 R-\mathrm{C} 3^{\prime} R\right)$

resulting in the meso-form

Scheme 6 Working hypothesis for copper catalyzed arylation-oxidative dimerization of $o$-haloanilides

being obtained as the major product and in a $50 \%$ yield (Scheme 8). It was noteworthy that this sequential arylation-oxidative dimerization process could be conducted on multi-gram scale in the presence of only catalytic amount of cuprous iodide $(10 \%)$. In order to determine the absolute configuration of compound 21a, we converted it to sulfonyl ester $\mathbf{2 3}$ and successfully obtained a suitable crystal for X-ray crystallography. The absolute configuration was confirmed by X-ray crystallography with Mo $\mathrm{K} \alpha$ radiation (Scheme 9).

Having successfully established the cascade process, we next initiated the synthesis of (+)-chimonanthine and its related natural dimeric HPI alkaloids. Based on our working hypothesis (Scheme 6), we used $(R)-(+)$-tert-butanesulfinamide as a chiral auxiliary. Treatment of 11a 


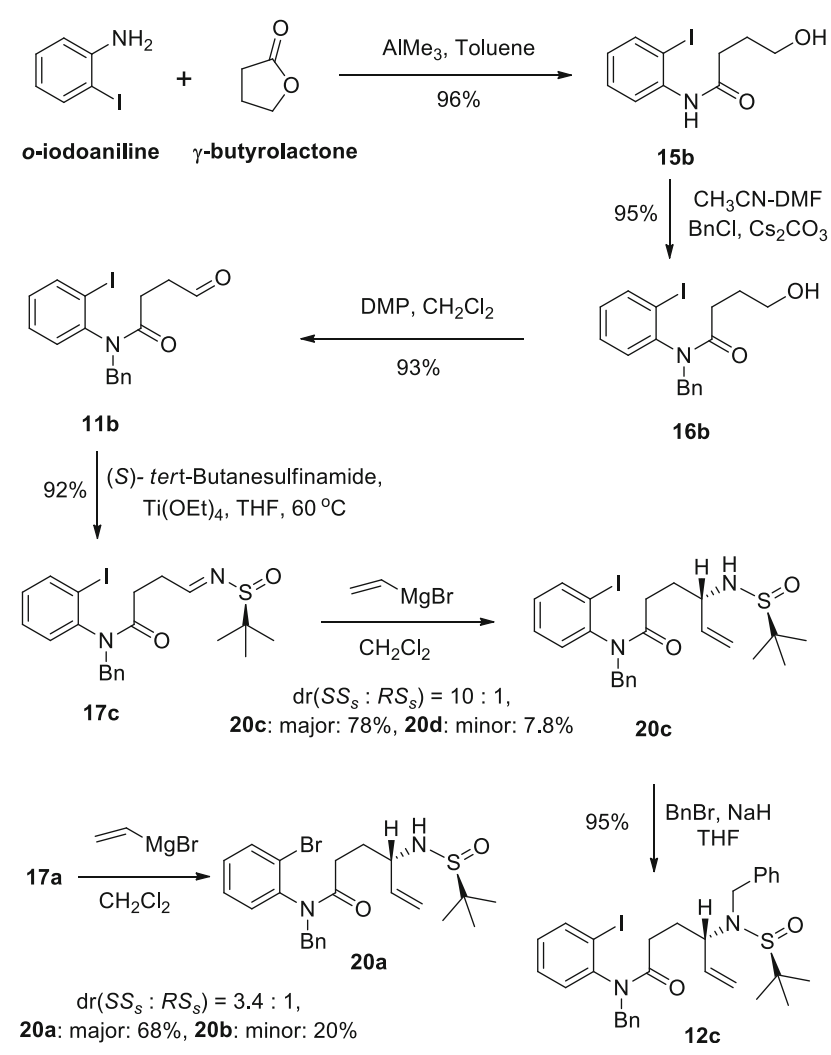

Scheme 7 Synthesis of amide 12c

with $(R)$ - $(+)$-tert-butanesulfinamide in the presence of

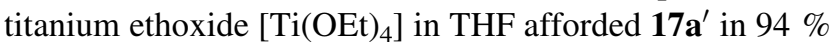
yield. After reduction and benzylation, amide $\mathbf{1 2} \mathbf{b}^{\prime}$ was synthesized in $68 \%$ overall yield from $o$-bromoaniline. The key arylation-oxidative dimerization of $\mathbf{1 2} \mathbf{b}^{\prime}$ (at multi-

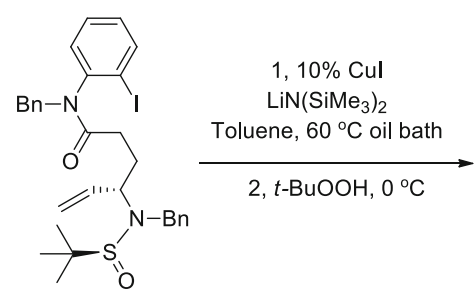

$12 \mathrm{c}$

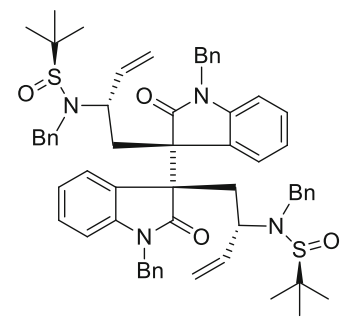

21b, Yield: $8 \%$

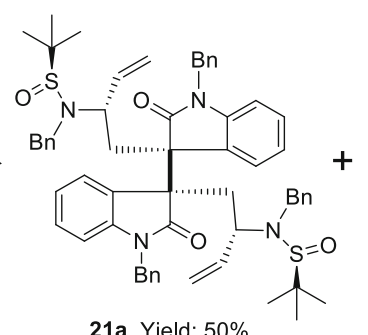

21a, Yield: $50 \%$

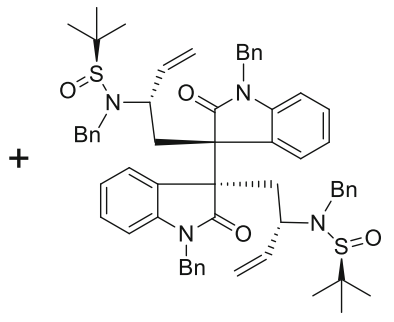

21c, Yield: $4 \%$
Scheme 8 Copper catalyzed sequential arylation-oxidative dimerization of $o$-bromoanilide 12c
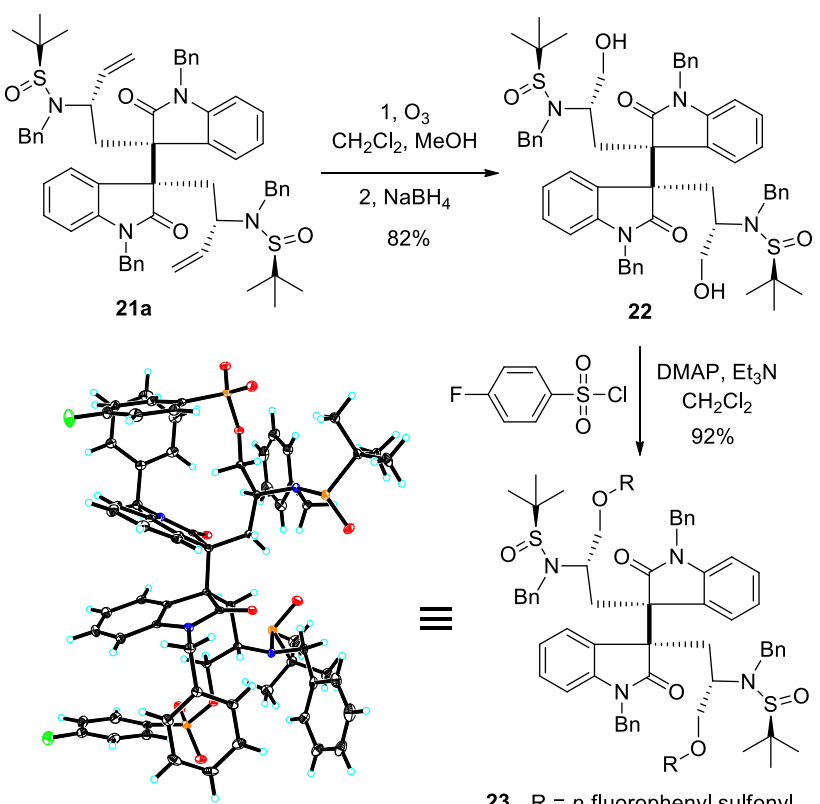

$23 \mathrm{R}=p$-fluorophenyl sulfonyl

Scheme 9 Determination of the absolute configuration of 21a by $\mathrm{X}$-ray crystallography with Mo $\mathrm{K} \alpha$ radiation

gram scale, $5.42 \mathrm{~g}, 10 \mathrm{mmol}$, see Scheme 10) afforded $\mathbf{1 3} \mathbf{a}^{\prime}+\mathbf{1 3} \mathbf{b}^{\prime}$ in $78 \%$ yield. Treatment of this diastereomeric mixture with $4 \mathrm{~N} \mathrm{HCl}$ in methanol gave diamine 24 in a $95 \%$ yield. After recrystallization in methanol with $2 \mathrm{~N}$ aqueous solution of $\mathrm{HCl}$ (2.0 eq.), the isomeric purity of di-amine $\mathbf{2 4}$ was greater than $99 \%$, as determined by HPLC analysis (Scheme 10, see supporting information). The reductive amination of $\mathbf{2 4}$ with formaldehyde and $\mathrm{NaBH}(\mathrm{OAc})_{3}$ provided diamide 25 (96\%) [19, 28]. Selective debenzylation of the amine benzyl group in $\mathbf{2 5}$ by treatment with $\alpha$-chloroethyl chloroformate (ACE-Cl) [77] followed by reflux in methanol afforded diamine $\mathbf{2 6}$ (95\%). Reductive aminocyclization of diamine 26 in THF in the presence of diisobutylaluminum hydride (DIBAL-H) provided desired HPI precursor (27) in $54 \%$ yield. Finally, removal of the benzyl protecting groups by a Birch reduction [19] afforded (+)-chimonanthine in $95 \%$ yield $\left([\alpha]_{\mathrm{D}}=+285, c 0.12, \mathrm{EtOH}\right.$, lit. [71], $[\alpha]_{\mathrm{D}}=+279, c 0.1$, EtOH, lit. [28], $[\alpha]_{\mathrm{D}}=+254, c 1.0$, EtOH). The NMR spectra of our synthetic sample were in fully agreement with those reported in the literature [19, 20, 28].

Treatment of chimonanthine with formaldehyde in the presence of sodium triacetoxyborohydride [28] then furnished (+)-folicanthine $\left(95 \%,[\alpha]_{\mathrm{D}}=+315, \quad c \quad 0.10\right.$, $\mathrm{MeOH}$, lit. [70], $[\alpha]_{\mathrm{D}}=+318, c 0.11, \mathrm{MeOH}$, lit. [71], $\left.[\alpha]_{\mathrm{D}}=+314, c 0.25, \mathrm{MeOH}\right)$. Upon exposure of chimonanthine to acid at reflux for $8 \mathrm{~h}[19,28](-)$-calycanthine $\left([\alpha]_{\mathrm{D}}=-615, \quad c 0.15, \mathrm{EtOH}\right.$, lit. $[28],[\alpha]_{\mathrm{D}}=-612$, $c 0.18, \mathrm{EtOH})$ was obtained in $52 \%$ isolated yield (Scheme 10). 

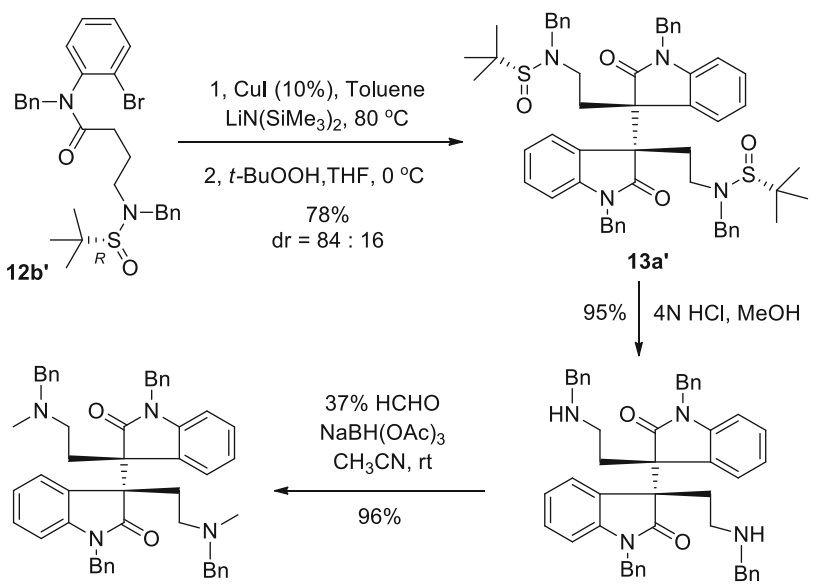

$$
95 \% \mid \begin{aligned}
& \mathrm{CH}_{3} \mathrm{CHClOCOCl} \\
& 1,2 \text {-dichloroethane, } 0-80^{\circ} \mathrm{C}, \\
& \text { then reflux in } \mathrm{MeOH}
\end{aligned}
$$

24, After recrystallization with $2 \mathrm{~N} \mathrm{HCl}$ (2 eq) in methanol, ee $>99 \%$, yield $=61 \%$
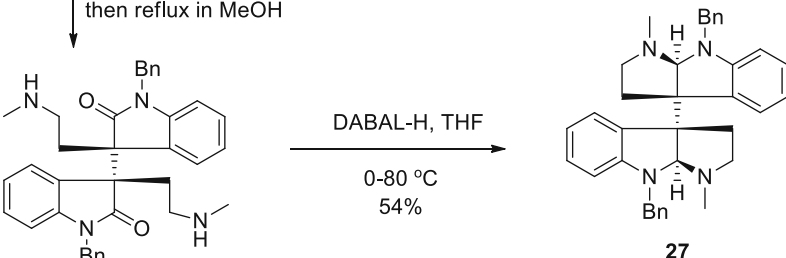

27

26
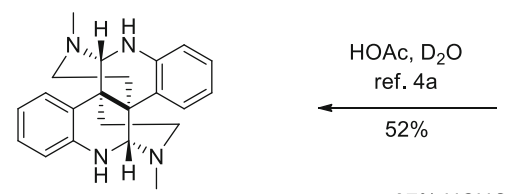

4

$37 \% \mathrm{HCHO}, \mathrm{CH}_{3} \mathrm{CN}$ $\mathrm{NaBH}(\mathrm{OAc})_{3}, 95 \%$

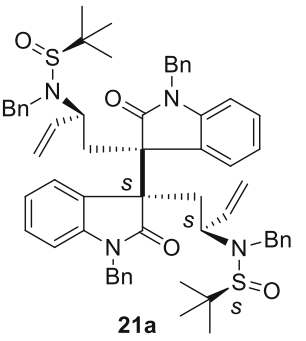

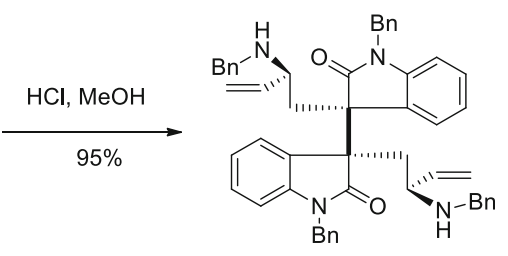

21

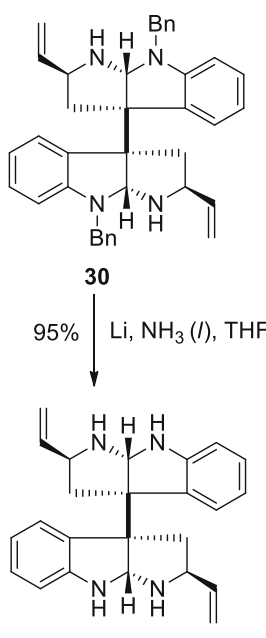

DABAL-H, THF $0-80^{\circ} \mathrm{C}$

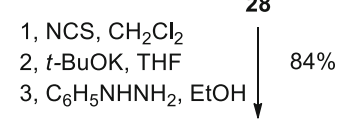

2, $t$-BuOK, THF $84 \%$

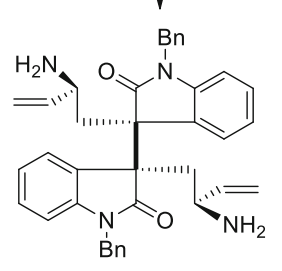

29
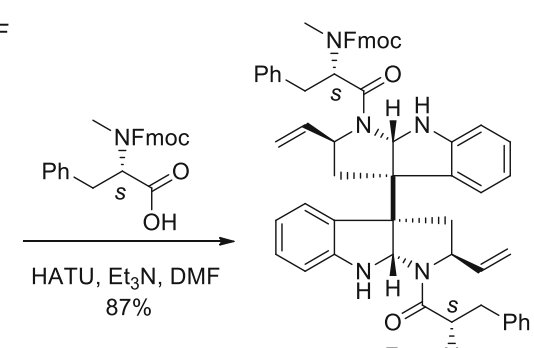

32 Fmocî̀

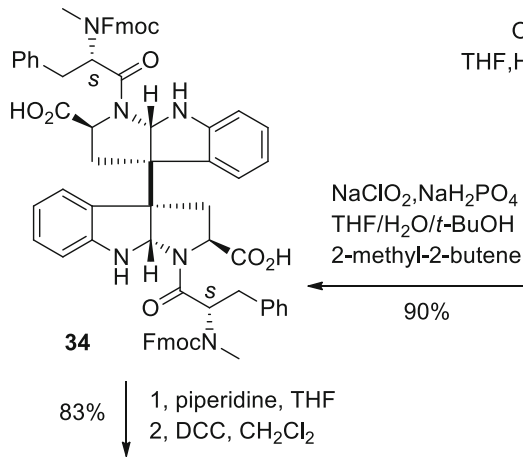

3: (-)-Ditryptophenaline

Starting from intermediate 21a, synthesis of (-)-ditryptophenaline was also investigated (Scheme 11). Removal of the tert-butanesulfinyl group by treatment of 21a with $4 \mathrm{~N} \mathrm{HCl}$ in methanol afforded diamine 28 . Selectively deprotection of the benzyl groups attaching to amines was difficult due to the presence of double bonds but finally achieved after many experiments. Treatment of diamine 28 with $N$-chlorosuccinamide, followed by deprotonation with $t$-BuOK provided imine [79], which, upon exposure to phenylhydrazine in ethanol [80], gave the primary diamine 29 in $84 \%$ overall yield. After reductive aminocyclization with DABAL-H and debenzylation with metal lithium in liquid ammonia (Scheme 11), intermediate 31 was obtained in $49 \%$ yield over two steps. Condensation of intermediate $\mathbf{3 1}$ with Fmoc-methylphenaline afforded compound 32 (FMOC-(S)-MePhe-OH, HATU and $\mathrm{Et}_{3} \mathrm{~N}$ in DMF) in $87 \%$ yield [33]. Cleavage of the double bond led to aldehyde $\mathbf{3 3}$, and oxidation with

Scheme 11 Total synthesis of (-)-ditryptophenaline

buffered $\mathrm{NaClO}_{2}$ provided diacid 34 [21]. Finally the diacid (34) was converted to (-)-ditryptophenaline $\left([\alpha]_{\mathrm{D}}=\right.$ $-292, c$ 0.40, $\mathrm{CH}_{2} \mathrm{Cl}_{2}$, lit. [29], $[\alpha]_{\mathrm{D}}=-292, c 0.97$, $\mathrm{CH}_{2} \mathrm{Cl}_{2}$, lit. [49], $[\alpha]_{\mathrm{D}}=-291, c 0.41, \mathrm{CH}_{2} \mathrm{Cl}_{2}$ ) in $83 \%$ overall yield by following Overman's procedure, deprotection with piperidine in THF and cyclization with DCC in dichloromethane (Scheme 11) [21]. The NMR spectra of our synthetic sample were in complete agreement with the reported data.

In conclusion, we have developed the first copper catalyzed arylation-oxidative dimerization of $o$-haloanilides with a remote assistance of an intramolecular sulfinyl amide unit. Based on this method, a general synthetic 
strategy has been successfully established for the total synthesis of chimonanthine, folicanthine, calycanthine and ditryptophenaline. This copper catalyzed sequential arylation-oxidative dimerization should find further application in the synthesis of HPI alkaloids as well as its medicinally interesting analogues.

\section{Experimental Section}

\subsection{General Experimental}

${ }^{1} \mathrm{H}-\mathrm{NMR}$ spectra were recorded on a Bruker Avance 300 or 400 spectrometer at 300 or $400 \mathrm{MHz}$. Carbon-13 nuclear magnetic resonance $\left({ }^{13} \mathrm{C}-\mathrm{NMR}\right)$ was recorded on Bruker Avance 300 or 400 spectrometer at 75 or $100 \mathrm{MHz}$. Chemical shifts are reported as $\delta$ values in parts per million (ppm) relative to tetramethylsilane for all recorded NMR spectra. Low-resolution mass spectra were recorded on a VG Auto Spec-3000 magnetic sector MS spectrometer. High resolution mass spectra were taken on AB QSTAR Pulsar mass spectrometer or Agilent G6230 TOF MS spectrometer. Chiral HPLC analyses were performed on Agilent 1100 series with a tunable UV detector at wavelength $\lambda=254 \mathrm{~nm}$. Melting points were determined on a capillary melting point apparatus and are uncorrected. Optical rotations were obtained on a UV-210A spectrometer. Starting materials and reagents used in reactions were obtained commercially from Acros, Aldrich, J\&K and were used without purification, unless otherwise indicated. THF and toluene used in the reactions were dried by distillation over metallic sodium; dichloromethane were distilled over $\mathrm{P}_{2} \mathrm{O}_{5}$. Unless otherwise stated, all reactions were conducted in dried glassware under a positive pressure of dry nitrogen or argon. Silica gel (Qingdao, 200-300 mesh) was used for column chromatography.

\subsection{Synthesis of Compound 15a}

2-Bromoaniline (12.04 g, $70.0 \mathrm{mmol})$ was dissolved in toluene $(200 \mathrm{~mL})$ under nitrogen. To this mixture, a solution of trimethylaluminum in toluene $(2.0 \mathrm{M}, 49 \mathrm{~mL}$, $98.0 \mathrm{mmol}, 1.4$ eq.) was added dropwise at $0{ }^{\circ} \mathrm{C}$. The resulting mixture was then stirred at room temperature for 45 min. $\gamma$-Butyrolactone (7.5 mL, $98 \mathrm{mmol}, 1.4$ eq.) was added via syringe and the reaction mixture was stirred at room temperature overnight. The solidified mixture was then cooled to $0{ }^{\circ} \mathrm{C}$, and $\mathrm{HCl}(1 \mathrm{~N}, 150 \mathrm{~mL})$ was added slowly. After $30 \mathrm{~min}$, the resulting mixture was extracted with ethyl acetate $(4 \times 80 \mathrm{~mL})$. The combined organic phases were washed with brine $(50 \mathrm{~mL})$ and dried over anhydrous sodium sulfate. After removal of the solvent, the crude products were chromatographed on silica gel (petroleum ether $60-90{ }^{\circ} \mathrm{C}$ : ethyl acetate $=1: 2$ ) to provide amide 15a (17.34 g, $96 \%)$ as a white solid.

m.p. $65-67^{\circ} \mathrm{C}$. $R_{\mathrm{f}} 0.55$ (petroleum ether:ethyl acetate =1:2). FTIR $\left(\mathrm{KBr}\right.$, thin film) $\mathrm{cm}^{-1} 3271,2943,2875$, $1660,1527,1430,1285,1051,755,673 .{ }^{1} H-N M R$ $\left(300 \mathrm{MHz}, \mathrm{CDCl}_{3}\right), \delta(\mathrm{ppm}) 8.27(1 \mathrm{H}, d, J=7.8 \mathrm{~Hz}), 7.81$ $(1 \mathrm{H}, \quad b r s), 7.53(1 \mathrm{H}, \quad d, \quad J=7.8 \mathrm{~Hz}), 7.30(1 \mathrm{H}, \quad t$, $J=7.8 \mathrm{~Hz}), 6.98(1 \mathrm{H}, \quad t, J=7.8 \mathrm{~Hz}), 3.75(2 \mathrm{H}, t$, $J=5.7 \mathrm{~Hz}), 2.59(3 \mathrm{H}, t, J=6.9 \mathrm{~Hz}), 2.05-1.91(2 \mathrm{H}, m)$. ${ }^{13} C-N M R \quad\left(75 \mathrm{MHz}, \mathrm{CDCl}_{3}\right) \delta(\mathrm{ppm}) 171.73,135.72$, $132.41,128.48,125.48,122.42,113.71,62.06,34.80$, 28.04. +TOF-MS $m / z(\%) 282\left(\mathrm{M}^{+}+1+\mathrm{Na}, 100 \%\right), 281$ $\left(\mathrm{M}^{+}+\mathrm{Na}, 8 \%\right), 280\left(\mathrm{M}^{+}+\mathrm{Na}, 96 \%\right), 260(42), 258(40)$, 240 (20), 200 (9), 174 (29), 172 (30). HRMS m/z Found 258.0124, Calcd. for $\mathrm{C}_{10} \mathrm{H}_{13} \mathrm{NO}_{2} \mathrm{Br}[\mathrm{M}+1]^{+} 258.0129$.

\subsection{Synthesis of Compound 16a}

To a mixture of cesium carbonate $(17.43 \mathrm{~g}, 53.5 \mathrm{mmol}$, 1.5 eq.) in acetonitrile $(90 \mathrm{~mL})$ and $N, N$-dimethylformamide (DMF, $45 \mathrm{~mL}$ ) at $0{ }^{\circ} \mathrm{C}$ was added dropwise a solution of amide 15a $(9.20 \mathrm{~g}, 35.7 \mathrm{mmol})$ in acetonitrile $(10 \mathrm{~mL})$ and DMF $(5 \mathrm{~mL})$. Benzyl bromide $(6.4 \mathrm{~mL}$, $53.5 \mathrm{mmol}, 1.5$ eq.) was then added. The resulting mixture was allowed to stir at room temperature for $8 \mathrm{~h}$. After filtration through a short column of silica gel and washed with ethyl acetate $(180 \mathrm{~mL})$, the combined organic phases were concentrated under reduced pressure and the residue was chromatographed on silica gel (petroleum ether 60-90 ${ }^{\circ} \mathrm{C}$ :ethyl acetate $\left.=1: 1\right)$ to afford alcohol 16a $(11.86 \mathrm{~g}, 96 \%)$ as a colorless oil.

$R_{\mathrm{f}} 0.60$ (petroleum ether:ethyl acetate $=1: 1$ ). FTIR (KBr, thin film) $\mathrm{cm}^{-1} 3417,2963,1651,1402,1270,1203$, 1050, 728. ${ }^{1} \mathrm{H}-\mathrm{NMR}\left(300 \mathrm{MHz}, \mathrm{CDCl}_{3}\right), \delta(\mathrm{ppm}) 7.68(1 \mathrm{H}$, $d d, J=2.7,7.8 \mathrm{~Hz}), 7.37-7.12(7 \mathrm{H}, m), 6.77(1 \mathrm{H}, d d$, $J=3.3,7.8 \mathrm{~Hz}), 5.64(1 \mathrm{H}, d, J=14.4 \mathrm{~Hz}), 4.01(1 \mathrm{H}, d$, $J=14.4 \mathrm{~Hz}), 3.71-3.55(2 \mathrm{H}, m), 2.89-2.68(1 \mathrm{H}, m), 2.14$ $(2 \mathrm{H}, \quad t, \quad J=6.6 \mathrm{~Hz}), \quad 1.98-1.78 \quad(2 \mathrm{H}, \quad m) .{ }^{13} C-N M R$ $\left(75 \mathrm{MHz}, \mathrm{CDCl}_{3}\right) \delta$ (ppm) 173.43, 140.71, 136.99, 134.07, $131.52,130.03,129.46,128.55,127.72,123.86,62.78$, $51.77,32.06,27.85$. +TOF-MS $\mathrm{m} / \mathrm{z}(\%) 372\left(\mathrm{M}^{+}+1+\mathrm{Na}\right.$, $99 \%), 371\left(\mathrm{M}^{+}+\mathrm{Na}, 9 \%\right), 370\left(\mathrm{M}^{+}+\mathrm{Na}, 100 \%\right), 350$ (32), 348 (34), 330 (2), 264 (3). HRMS m/z Found 370.0410, Calcd. for $\mathrm{C}_{17} \mathrm{H}_{18} \mathrm{NO}_{2} \mathrm{NaBr}[\mathrm{M}+23]^{+} 370.0418$.

\subsection{Synthesis of Compound 11a}

Alcohol 16a (11.86 g, $34.1 \mathrm{mmol})$ was dissolved in dichloromethane $(120 \mathrm{~mL})$. To this solution, a powder of Dess-Martin periodinane $(21.67 \mathrm{~g}, 51.1 \mathrm{mmol}, 1.5 \mathrm{eq}$. was added. The resulting mixture was then stirred at room temperature for $6 \mathrm{~h}$. After filtration through a short column of silica gel and washed with ethyl acetate $(150 \mathrm{~mL})$, the 
combined organic phases were concentrated under reduced pressure and the residue was chromatographed on silica gel (petroleum ether $60-90{ }^{\circ} \mathrm{C}$ :ethyl acetate $=3: 1$ ) to afford aldehyde 11a (10.85 g, $92 \%)$ as white plates.

m.p. $52-54^{\circ} \mathrm{C} . R_{\mathrm{f}} 0.53$ (petroleum ether:ethyl acetate $=3: 1)$. FTIR $\left(\mathrm{KBr}\right.$, thin film) $\mathrm{cm}^{-1} 3419,3060,2921$, 2827, 2730, 1657, 1400, 1265, 1196, 1020, 727. ${ }^{1} H-N M R$ $\left(300 \mathrm{MHz}, \mathrm{CDCl}_{3}\right), \delta(\mathrm{ppm}) 9.81(1 \mathrm{H}, s), 7.72-7.62(1 \mathrm{H}$, $m), 7.38-7.12(7 \mathrm{H}, m), 6.92-6.82(1 \mathrm{H}, m), 5.62(1 \mathrm{H}, d$, $J=14.4 \mathrm{~Hz}), 4.03(1 \mathrm{H}, d, J=14.4 \mathrm{~Hz}), 2.91(1 \mathrm{H}, d d d$, $J=6.9,7.8,18.6 \mathrm{~Hz}), 2.68(1 \mathrm{H}, d t, J=6.3,18.6 \mathrm{~Hz})$, $2.42-2.16(2 \mathrm{H}, m) .{ }^{13} \mathrm{C}-\mathrm{NMR}\left(75 \mathrm{MHz}, \mathrm{CDCl}_{3}\right), \delta(\mathrm{ppm})$ 200.99, 171.12, 140.45, 136.88, 133.99, 131.60, 130.08, $129.29,128.62,128.50,127.64,123.87,51.75,38.98$, 27.18. +TOF-MS $\mathrm{m} / \mathrm{z}$ (\%) $370\left(\mathrm{M}^{+}+1+\mathrm{Na}, 98 \%\right)$, $369\left(\mathrm{M}^{+}+\mathrm{Na}, 13 \%\right), 368\left(\mathrm{M}^{+}+\mathrm{Na}, 100 \%\right), 348(81)$, 346 (90), 332 (30), 330 (31), 262 (3). HRMS m/z Found 368.0266, Calcd. for $\mathrm{C}_{17} \mathrm{H}_{16} \mathrm{NO}_{2} \mathrm{NaBr}[\mathrm{M}+\mathrm{Na}]^{+}$ 368.0262 .

\subsection{Synthesis of Compound $\mathbf{1 7 b}$}

To a solution of aldehyde 11a $(5.85 \mathrm{~g}, 16.8 \mathrm{mmol})$ in toluene $(60 \mathrm{~mL})$ was added a powder of $(S)-(-)$-tert-butanesulfinamide (4.1 g, $33.7 \mathrm{mmol}, 2.0$ eq.) and $\mathrm{KHSO}_{4}$ ( $4.56 \mathrm{~g}, 33.7 \mathrm{mmol}, 2.0$ eq.). The resulting mixture was stirred at $45{ }^{\circ} \mathrm{C}$ for $3 \mathrm{~h}$. After filtration through a short column of silica gel and washed with ethyl acetate $(80 \mathrm{~mL})$, the combined organic phases were concentrated under reduced pressure and the residue was flash chromatographed on silica gel (petroleum ether $60-90{ }^{\circ} \mathrm{C}$ :ethyl acetate $=3: 1)$ to afford the sulfinyl imine $(17 \mathbf{a}: 7.36 \mathrm{~g}$, $97 \%)$ as colorless syrup. The sulfinyl imine was re-dissolved in anhydrous methanol $(60 \mathrm{~mL})$ and the resulting solution was cooled to $0{ }^{\circ} \mathrm{C}$. A powder of sodium borohydride ( $1.87 \mathrm{~g}, 49.2 \mathrm{mmol}, 3.0$ eq.) was added in small portion over a period of $30 \mathrm{~min}$. The resulting mixture was then allowed to stir at $0{ }^{\circ} \mathrm{C}$ for $4 \mathrm{~h}$. After which, a saturated solution of $\mathrm{NH}_{4} \mathrm{Cl}(20 \mathrm{~mL})$ was introduced and the resulting mixture was concentrated (ca. $20-30 \mathrm{~mL}$ ). The mixture was diluted with water $(100 \mathrm{~mL})$ and extracted with ethyl acetate $(4 \times 50 \mathrm{~mL})$. The combined organic phases were washed with brine $(60 \mathrm{~mL})$ and dried over anhydrous sodium sulfate. After removal of the solvent, the crude products were chromatographed on silica gel (petroleum ether $60-90{ }^{\circ} \mathrm{C}$ :ethyl acetate $=1: 2$ ) to provide sulfinamide $17 \mathrm{~b}(6.6 \mathrm{~g}, 89 \%)$ as a white solid.

m.p. $102-103{ }^{\circ} \mathrm{C} .[\alpha]_{\mathrm{D}}^{20}+71\left(\mathrm{c} 0.90, \mathrm{CHCl}_{3}\right) \cdot R_{\mathrm{f}} 0.45$ (petroleum ether:ethyl acetate $=1: 2)$. FTIR $(\mathrm{KBr}$, thin film) $\mathrm{cm}^{-1} 3244,3063,2950,2868,1662,1559,1468$, $1398,1266,1203,1063,947,767,732,628 .{ }^{1} H-N M R$ (as a mixture of rotamers, $300 \mathrm{MHz}, \mathrm{CDCl}_{3}$ ), $\delta$ (ppm) 7.71-7.61
$(1 \mathrm{H}, m), 7.31-7.12(7 \mathrm{H}, m), 6.81-6.70(1 \mathrm{H}, m), 5.58(1 \mathrm{H}$, $d, J=14.1 \mathrm{~Hz}), 4.00(0.5 \mathrm{H}, d, J=14.1 \mathrm{~Hz})[3.99(0.5 \mathrm{H}$, $d, J=14.1 \mathrm{~Hz})], 3.42-3.23(1 \mathrm{H}, m), 3.22-3.10(1 \mathrm{H}, m)$, 3.10-2.94 (1H, $m), 2.11-1.97(2 \mathrm{H}, m), 1.93-1.78(2 \mathrm{H}, m)$, $1.13(9 \mathrm{H}, s) .{ }^{13} \mathrm{C}-\mathrm{NMR}$ (rotamer in brackets, $75 \mathrm{MHz}$, $\left.\mathrm{CDCl}_{3}\right), \delta(\mathrm{ppm}) 172.16,140.67,137.02,134.00$ (133.94), 131.57 (131.50), 129.94, 129.41, 128.47, 127.63, 123.85, 55.64, 51.58, 45.07 (44.99), 31.62, 26.15, 22.71. +TOFMS $m / z(\%) 475\left(\mathrm{M}^{+}+1+\mathrm{Na}, 60 \%\right), 474\left(\mathrm{M}^{+}+\mathrm{Na}, 8 \%\right)$, $473\left(\mathrm{M}^{+}+\mathrm{Na}, 65 \%\right), 453$ (98), 452 (12), 451 (100), 435 (25), 433 (23), 402 (4), 400 (4), 294 (2), 282 (6), 280 (5), 262 (2). HRMS m/z Found 473.0878, Calcd. for $\mathrm{C}_{21} \mathrm{H}_{27} \mathrm{~N}_{2} \mathrm{O}_{2} \mathrm{NaSBr}[\mathrm{M}+\mathrm{Na}]^{+} 473.0874$.

\subsection{Synthesis of Compound 12a}

To a mixture of sodium hydride $(60 \%$ in mineral oil, $1.22 \mathrm{~g}, 30.5 \mathrm{mmol}$, freshly washed with anhydrous hexane three times under nitrogen) in anhydrous THF $(50 \mathrm{~mL})$ at $0{ }^{\circ} \mathrm{C}$ was added a solution of sulfonamide $17 \mathrm{~b}(10.6 \mathrm{~g}$, $23.5 \mathrm{mmol})$ in THF $(50 \mathrm{~mL})$ via syringe. After stirring at $0{ }^{\circ} \mathrm{C}$ for $30 \mathrm{~min}$, methyl iodide $(1.9 \mathrm{~mL}, 30.6 \mathrm{mmol})$ was added. The resulting mixture was then stirred at room temperature for $15 \mathrm{~h}$ under nitrogen. A powder of $\mathrm{NH}_{4} \mathrm{Cl}$ $(1.62 \mathrm{~g}, 30.0 \mathrm{mmol})$ was added and the mixture was stirred for $10 \mathrm{~min}$. After concentrated under reduced pressure, the residue was diluted with water $(100 \mathrm{~mL})$ and extracted with ethyl acetate $(3 \times 50 \mathrm{~mL})$. The combined organic phases were washed with brine $(20 \mathrm{~mL})$ and dried over anhydrous $\mathrm{Na}_{2} \mathrm{SO}_{4}$. After filtration, the solvent was removed under reduced pressure and the residue was chromatographed on silica gel (petroleum ether $60-90{ }^{\circ} \mathrm{C}$ :ethyl acetate $=1: 1$ ) to afford the product (12a: $10.03 \mathrm{~g}, 92 \%)$ as a white solid.

m.p. $60-62{ }^{\circ} \mathrm{C}$. $R_{\mathrm{f}} 0.52$ (petroleum ether:ethyl acetate $=1: 1) . \quad{ }^{l} \mathrm{H}-\mathrm{NMR} \quad\left(300 \mathrm{MHz}, \quad \mathrm{CDCl}_{3}\right), \quad \delta \quad(\mathrm{ppm})$ 7.65-7.58 $(1 \mathrm{H}, m), 7.28-7.08(7 \mathrm{H}, m), 6.78-6.68(1 \mathrm{H}, m)$, $5.57 \quad(1 \mathrm{H}, \quad d d, \quad J=2.1, \quad 14.4 \mathrm{~Hz}), \quad 3.95 \quad(1 \mathrm{H}, \quad d$, $J=14.4 \mathrm{~Hz}), 3.01-2.82(2 \mathrm{H}, m), 2.52(3 \mathrm{H}, s), 2.01-1.78$ $(4 \mathrm{H}, m), 1.09(9 \mathrm{H}, s) .{ }^{13} \mathrm{C}-\mathrm{NMR}\left(75 \mathrm{MHz}, \mathrm{CDCl}_{3}\right), \delta(\mathrm{ppm})$ $171.77,140.51,136.93,133.86,131.42,129.86,129.24$, $128.43,128.36,127.51,123.76,58.11,53.94$ (53.57), 51.41, 32.65 (32.48), 23.64, 23.43. +TOF-MS $m / z$ (\%) 489 $\left(\mathrm{M}^{+}+1+\mathrm{Na}, 100 \%\right), 488\left(\mathrm{M}^{+}+\mathrm{Na}, 12 \%\right), 487\left(\mathrm{M}^{+}+\mathrm{Na}\right.$, $98 \%), 467$ (18), 465 (16), 432 (2), 383 (1), 375 (2), 361 (21), 359 (20), 276 (3), 274 (2). HRMS m/z Found 487.1023, Calcd. for $\mathrm{C}_{22} \mathrm{H}_{29} \mathrm{~N}_{2} \mathrm{O}_{2} \mathrm{NaSBr} \quad[\mathrm{M}+\mathrm{Na}]^{+}$ 487.1030 .

\subsection{Synthesis of Compound $\mathbf{1 2 b}$}

To a mixture of sodium hydride $60 \%$ in mineral oil, $1.32 \mathrm{~g}, 33 \mathrm{mmol}, 1.5$ eq., freshly washed with anhydrous 
hexane three times under nitrogen) in anhydrous THF $(30 \mathrm{~mL})$ at $0{ }^{\circ} \mathrm{C}$ was added a solution of sulfonamide $\mathbf{1 7 b}$ $(9.9 \mathrm{~g}, 22 \mathrm{mmol})$ in THF $(80 \mathrm{~mL})$ via syringe. After stirring at $0{ }^{\circ} \mathrm{C}$ for $30 \mathrm{~min}$, benzyl bromide $(3.9 \mathrm{~mL}, 33 \mathrm{mmol}$, 1.5 eq.) was added. The resulting mixture was then stirred at $0{ }^{\circ} \mathrm{C}$ for $2 \mathrm{~h}$, then at room temperature for $12 \mathrm{~h}$ under nitrogen. A powder of $\mathrm{NH}_{4} \mathrm{Cl}(1.62 \mathrm{~g}, 30.0 \mathrm{mmol})$ was added and the mixture was stirred for $10 \mathrm{~min}$. After concentrated under reduced pressure, the residue was diluted with water $(60 \mathrm{~mL})$ and extracted with ethyl acetate $(3 \times 50 \mathrm{~mL})$. The combined organic phases were washed with brine $(30 \mathrm{~mL})$ and dried over anhydrous $\mathrm{Na}_{2} \mathrm{SO}_{4}$. After filtration, the solvent was removed under reduced pressure and the residue was chromatographed on silica gel (petroleum ether $60-90{ }^{\circ} \mathrm{C}$ :ethyl acetate $=2: 1$ ) to afford the product (12b: $11.2 \mathrm{~g}, 94 \%$ ) as an off-yellow oil.

$[\alpha]_{\mathrm{D}}^{20}-3.9\left(\mathrm{c} 0.40, \mathrm{CHCl}_{3}\right) . R_{\mathrm{f}} 0.51$ (petroleum ether:ethyl acetate $=2: 1)$. FTIR $\left(\mathrm{KBr}\right.$, thin film $\mathrm{cm}^{-1} 3421$, 2960, 1665, 1467, 1396, 1278, 1204, 1070, 1024, 928, 700. ${ }^{1} \mathrm{H}-\mathrm{NMR}$ (as a mixture of rotamers, $300 \mathrm{MHz}, \mathrm{CDCl}_{3}$ ), $\delta$ (ppm) 7.71-7.61 $(1 \mathrm{H}, m), 7.37-7.11(12 \mathrm{H}, m), 6.77-6.67$ $(1 \mathrm{H}, m), 5.59(0.5 \mathrm{H}, d, J=14.4 \mathrm{~Hz})[5.59(0.5 \mathrm{H}, d, J=$ $14.1 \mathrm{~Hz})], 4.26(1 \mathrm{H}, d, J=15.6 \mathrm{~Hz}), 4.10(0.5 \mathrm{H}, d$, $J=15.6 \mathrm{~Hz})[4.08(0.5 \mathrm{H}, d, J=15.3 \mathrm{~Hz})], 3.98(0.5 \mathrm{H}, d$, $J=14.1 \mathrm{~Hz})[3.97(0.5 \mathrm{H}, d, J=14.4 \mathrm{~Hz})], 3.03-2.85$ $(1 \mathrm{H}, m), 2.84-2.68(1 \mathrm{H}, m), 1.97-1.76(4 \mathrm{H}, m), 1.16(9 \mathrm{H}, s)$. ${ }^{13} \mathrm{C}$-NMR (rotamer in brackets, $75 \mathrm{MHz}, \mathrm{CDCl}_{3}$ ), $\delta(\mathrm{ppm})$ $171.69,140.56,137.24,137.02,133.92,131.52,131.45$, $129.88,129.32,128.54,128.44,127.57,127.35,123.85$, 58.22, 51.47 (51.07), 48.23 (47.92), 31.79 (31.71), 23.93 (23.81), 23.39. EI-MS $m / z$ (\%) $543\left(\mathrm{M}^{+}+2,6 \%\right), 541$ $\left(\mathrm{M}^{+}+1,6 \%\right), 486(10), 486(34), 484(30), 430$ (25), 395 (26), 330 (16), 263 (31), 261 (30), 212 (17), 174 (16), 147 (11), 91 (48), 90 (100), 85 (4), 76 (12), 57 (29). HRMS m/ $z$ Found 540.1448, Calcd. for $\mathrm{C}_{28} \mathrm{H}_{33} \mathrm{~N}_{2} \mathrm{O}_{2} \mathrm{SBr}[\mathrm{M}]^{+}$ 540.1446 .

\subsection{Synthesis of Compound $\mathbf{1 5 b}$}

2-Iodoaniline $(21.9 \mathrm{~g}, 100.0 \mathrm{mmol})$ was dissolved in toluene $(300 \mathrm{~mL})$ under nitrogen. To this mixture, a solution of trimethylaluminum in toluene $(2.0 \mathrm{M}, 60 \mathrm{~mL}$, $120 \mathrm{mmol}, 1.2$ eq.) was added dropwise at $0{ }^{\circ} \mathrm{C}$. The resulting mixture was then stirred at room temperature for $45 \mathrm{~min}$, after which, $\gamma$-butyrolactone $(9.2 \mathrm{~mL}, 120 \mathrm{mmol}$, 1.2 eq.) was added via syringe and the reaction mixture was stirred at room temperature overnight. The solidified mixture was then cooled to $0{ }^{\circ} \mathrm{C}$ and $\mathrm{HCl}(1 \mathrm{~N}, 360 \mathrm{~mL})$ was added slowly. After $1 \mathrm{~h}$, the resulting mixture was extracted with ethyl acetate $(4 \times 150 \mathrm{~mL})$. The combined organic phases were washed with brine $(100 \mathrm{~mL})$ and dried over anhydrous sodium sulfate. After removal of the solvent, the crude products were chromatographed on silica gel (petroleum ether $60-90{ }^{\circ} \mathrm{C}$ :ethyl acetate $=1: 2$ ) to provide amide $\mathbf{1 5 b}(29.30 \mathrm{~g}, 96 \%)$ as a white solid.

m.p. $65-66{ }^{\circ} \mathrm{C} . R_{\mathrm{f}} 0.59$ (petroleum ether:ethyl acetate $=1: 2)$. FTIR $(\mathrm{KBr}$, thin film $) \mathrm{cm}^{-1} 3268,2935,1658$, 1528, 1430, 1287, 1059, 749, 664. ${ }^{1} H-N M R$ (400 MHz, $\left.\mathrm{CDCl}_{3}\right), \delta(\mathrm{ppm}) 8.02(1 \mathrm{H}, d, J=8.0 \mathrm{~Hz}), 7.76(1 \mathrm{H}, s)$, $7.75(1 \mathrm{H}, d, J=8.0 \mathrm{~Hz}), 7.30(1 \mathrm{H}, t, J=7.6 \mathrm{~Hz}), 6.83$ $(1 \mathrm{H}, t, J=7.6 \mathrm{~Hz}), 3.72(2 \mathrm{H}, t, J=6.0 \mathrm{~Hz}), 3.32(1 \mathrm{H}$, $b r s), 2.56(3 \mathrm{H}, t, J=6.9 \mathrm{~Hz}), 2.05-1.91(2 \mathrm{H}, m) .{ }^{13} C-$ NMR $\left(100 \mathrm{MHz}, \mathrm{CDCl}_{3}\right) \delta(\mathrm{ppm}) 171.92,138.90,138.16$, $129.16,126.43,123.11,91.14,61.76,34.52$, 28.06. EI-MS $\mathrm{m} / \mathrm{z}(\%) 304\left(\mathrm{M}^{+}, 28 \%\right), 268(89 \%), 224(12 \%), 182$ (30), 180 (40), 152 (10), 128 (6), 104 (12), 91 (100). HRMS $\mathrm{m} / \mathrm{z}$ Found 304.9969, Calcd. for $\mathrm{C}_{10} \mathrm{H}_{12} \mathrm{NO}_{2} \mathrm{I}[\mathrm{M}]^{+}$ 304.9913 .

\subsection{Synthesis of Compound $\mathbf{1 6 b}$}

To a mixture of amide $\mathbf{1 5 b}(29.3 \mathrm{~g}, 96 \mathrm{mmol})$, cesium carbonate $(46.9 \mathrm{~g}, 144 \mathrm{mmol}, 1.5$ eq.) in acetonitrile $(200 \mathrm{~mL})$ and $N, N$-dimethylformamide (DMF, $100 \mathrm{~mL}$ ) at $0{ }^{\circ} \mathrm{C}$ was added dropwise a solution of benzyl bromide $(17.1 \mathrm{~mL}, 144 \mathrm{mmol}, 1.5$ eq.) was then added. The resulting mixture was then stirred at room temperature for $12 \mathrm{~h}$. After filtration through a short column of silica gel and washed with ethyl acetate $(200 \mathrm{~mL})$, the combined organic phases were concentrated under reduced pressure and the residue was chromatographed on silica gel (petroleum ether $60-90{ }^{\circ} \mathrm{C}$ :ethyl acetate $=1: 1$ ) to afford alcohol 16b (36.03 g, $95 \%)$ as a white powder.

m.p. $72-74{ }^{\circ} \mathrm{C} . R_{\mathrm{f}} 0.55$ (petroleum ether:ethyl acetate $=1: 1)$. FTIR $(\mathrm{KBr}$, thin film $) \mathrm{cm}^{-1} 3500,2940,2871$, 1631, 1405, 1322, 1051, 1008, 731, 616, 491. ${ }^{1} \mathrm{H}-\mathrm{NMR}$ $\left(400 \mathrm{MHz}, \mathrm{CDCl}_{3}\right), \delta(\mathrm{ppm}) 7.93(1 \mathrm{H}, d d, J=1.2$, $8.0 \mathrm{~Hz}), 7.28-7.15(6 \mathrm{H}, m), 7.03(1 \mathrm{H}, d d d, J=1.2,7.8$, $8.0 \mathrm{~Hz}), 6.71(1 \mathrm{H}, d d, J=1.2,7.8 \mathrm{~Hz}), 5.67(1 \mathrm{H}, d$, $J=14.4 \mathrm{~Hz}), 3.91(1 \mathrm{H}, d, J=14.4 \mathrm{~Hz}), 3.70-3.53(2 \mathrm{H}$, $m), 3.12-3.04(1 \mathrm{H}, m), 2.10(2 \mathrm{H}, t, J=6.6 \mathrm{~Hz}), 1.92-1.78$ $(2 \mathrm{H}, m) .{ }^{13} C-N M R\left(100 \mathrm{MHz}, \mathrm{CDCl}_{3}\right) \delta$ (ppm) 173.18, $143.80,140.31,136.83,130.83,130.00,129.43,129.35$, $128.47,127.65,100.36,62.51,51.81,32.23,27.79$. EI-MS $\mathrm{m} / \mathrm{z}(\%) 395\left(\mathrm{M}^{+}, 10 \%\right), 351(3 \%), 309$ (20\%), 224 (4), 203 (5), 182 (15), 180 (30), 152 (6), 134 (9), 119 (17), 104 (7), 91 (100), 77 (14). HRMS m/z Found 395.0378, Calcd. for $\mathrm{C}_{17} \mathrm{H}_{18} \mathrm{NO}_{2} \mathrm{I}[\mathrm{M}]^{+} 395.0382$.

\subsection{Synthesis of Compound $\mathbf{1 1 b}$}

Alcohol 16b (36.0 g, $91.1 \mathrm{mmol})$ was dissolved in dichloromethane $(300 \mathrm{~mL})$. To this solution, a powder of Dess-Martin periodinane (46.4 g, $109.3 \mathrm{mmol}, 1.2 \mathrm{eq}$.) was added. The resulting mixture was then stirred at room 
temperature for $3 \mathrm{~h}$. After filtration through a short column of silica gel and washed with ethyl acetate $(150 \mathrm{~mL})$, the combined organic phases were concentrated and the residue was chromatographed on silica gel (petroleum ether $60-90{ }^{\circ} \mathrm{C}$ :ethyl acetate $=3: 1$ ) to afford aldehyde $\mathbf{1 1 b}$ $(32.94 \mathrm{~g}, 92 \%)$ as pale yellow plates.

m.p. $76-78^{\circ} \mathrm{C} . R_{\mathrm{f}} 0.53$ (petroleum ether:ethyl acetate $=3: 1)$. FTIR $\left(\mathrm{KBr}\right.$, thin film) $\mathrm{cm}^{-1} 3433,3056,2826$, 1713, 1651, 1400, 1268, 1017, 771, 724. ${ }^{1} H-N M R$ $\left(400 \mathrm{MHz}, \mathrm{CDCl}_{3}\right), \delta(\mathrm{ppm}) 9.77(1 \mathrm{H}, s), 7.93(1 \mathrm{H}, d d$, $J=1.2,8.0 \mathrm{~Hz}), 7.29-7.13(6 \mathrm{H}, m), 7.01(1 \mathrm{H}, d d d$, $J=1.2,7.8,8.0 \mathrm{~Hz}), 6.83(1 \mathrm{H}, d d, J=1.2,7.8 \mathrm{~Hz}), 5.65$ $(1 \mathrm{H}, d, J=14.4 \mathrm{~Hz}), 3.92(1 \mathrm{H}, d, J=14.4 \mathrm{~Hz}), 2.86(1 \mathrm{H}$, $d d d, \quad J=6.0,7.6,18.7 \mathrm{~Hz}), 2.65(1 \mathrm{H}, d t, \quad J=6.0$, $18.7 \mathrm{~Hz}), 2.32(1 \mathrm{H}, d d d, J=6.0,7.6,17.1 \mathrm{~Hz}), 2.18(1 \mathrm{H}$, $d t, J=6.0,17.1 \mathrm{~Hz}) .{ }^{13} C-N M R\left(100 \mathrm{MHz}, \mathrm{CDCl}_{3}\right), \delta$ (ppm) 200.64, 170.60, 143.34, 139.98, 136.49, 130.65, 129.86, 129.21, 128.99, 128.18, 127.33, 100.11, 51.54, 38.57, 27.26. EI-MS m/z (\%) $393\left(\mathrm{M}^{+}, 12 \%\right), 369$ (3\%), 351 (3\%), 309 (28), 282 (5), 268 (70), 230 (4), 224 (4), 203 (9), 182 (23), 180 (63), 152 (12), 104 (7), 91 (100), 77 (13). HRMS m/z Found 393.0265, Calcd. for $\mathrm{C}_{17} \mathrm{H}_{16} \mathrm{NO}_{2} \mathrm{I}$ $[\mathrm{M}]^{+} 393.0226$.

\subsection{Synthesis of Compound 17c}

To a solution of aldehyde $\mathbf{1 1 b}(19.7 \mathrm{~g}, 50 \mathrm{mmol})$ in THF $(200 \mathrm{~mL})$ was added a powder of $(S)-(-)$-tert-butanesulfinamide $\left(12.1 \mathrm{~g}, 100 \mathrm{mmol}, 2.0 \mathrm{eq}\right.$.) and $\mathrm{Ti}(\mathrm{OEt})_{4}(22.8 \mathrm{~g}$, $100 \mathrm{mmol}, 2.0$ eq.). The resulting mixture was stirred at $60{ }^{\circ} \mathrm{C}$ under nitrogen for $12 \mathrm{~h}$. The reaction mixture was then cooled to room temperature and treated with saturated $\mathrm{NaCl}$ aqueous solution $(100 \mathrm{~mL})$ for $1 \mathrm{~h}$. After filtration through a short column of Celite and washed with ethyl acetate $(80 \mathrm{~mL})$, the combined organic phases were dried over anhydrous $\mathrm{Na}_{2} \mathrm{SO}_{4}$. After removal of the solvent under reduced pressure, the residue was flash chromatographed on silica gel (petroleum ether $60-90{ }^{\circ} \mathrm{C}$ :ethyl acetate $=3: 1)$ to afford the sulfinyl imine $(\mathbf{1 7 c}: 22.85 \mathrm{~g}$, $92 \%)$ as a pale yellow syrup.

$[\alpha]_{\mathrm{D}}^{20}+52\left(\mathrm{c} \quad 0.19, \mathrm{CHCl}_{3}\right) \cdot R_{\mathrm{f}} 0.51$ (petroleum ether:ethyl acetate $=3: 1)$. FTIR $(\mathrm{KBr}$, thin film $) \mathrm{cm}^{-1} 3445$, 2964, 1663,1466, 1400, 1079. ${ }^{1} H-N M R$ (as a mixture of rotamers, $\left.400 \mathrm{MHz}, \mathrm{CDCl}_{3}\right), \delta(\mathrm{ppm}) 8.09(1 \mathrm{H}$, brs $), 7.93$ $(1 \mathrm{H}, d, J=8.0 \mathrm{~Hz}), 7.28-7.14(6 \mathrm{H}, m), 7.08-7.01(1 \mathrm{H}, m)$, $6.75(0.5 \mathrm{H}, d, J=7.6 \mathrm{~Hz})[6.74(0.5 \mathrm{H}, d, J=7.6 \mathrm{~Hz})]$, $5.67(0.5 \mathrm{H}, d, J=14.4 \mathrm{~Hz})[5.59(0.5 \mathrm{H}, d, J=14.0 \mathrm{~Hz})]$, $3.97(0.5 \mathrm{H}, d, J=14.0 \mathrm{~Hz})[3.92(0.5 \mathrm{H}, d, J=14.4 \mathrm{~Hz})]$, $3.00-2.87(1 \mathrm{H}, m), 2.81-2.68(1 \mathrm{H}, m), 2.46-2.18(2 \mathrm{H}, m)$, $1.14(4.5 \mathrm{H}, s)[1.12(4.5 \mathrm{H}, s)] .{ }^{13} C-N M R$ (rotamer in brackets, $100 \mathrm{MHz}, \mathrm{CDCl}_{3}$ ), $\delta$ (ppm) 170.51 (170.39),
168.11 (168.06), 143.54, 140.20 (140.13), 136.69 (136.60), 130.76 (130.65), 129.89, 129.45 (129.24), 129.13, 128.24, 127.45 (127.42), 100.24, 56.57 (56.53), 51.71 (51.55), 31.13 (30.99), 29.88 (29.83), 22.25 (22.22). EI-MS m/ z (\%) $496\left(\mathrm{M}^{+}, 10 \%\right), 395$ (3), 378 (16), 309 (10), 298 (15), 268 (20), 253 (4), 224 (2), 201 (7), 182 (11), 180 (20), 152 (5), 133 (21), 119 (46), 103 (6), 91 (100), 77 (14). HRMS m/z Found 496.0663, Calcd. for $\mathrm{C}_{21} \mathrm{H}_{25} \mathrm{~N}_{2} \mathrm{O}_{2} \mathrm{SI}$ $[\mathrm{M}]^{+} 496.0682$.

\subsection{Synthesis of Compound 20c}

A solution of imine 17c $(19.8 \mathrm{~g}, 40 \mathrm{mmol})$ in dichloromethane $(200 \mathrm{~mL})$ was stirred at $-78{ }^{\circ} \mathrm{C}$ for $10 \mathrm{~min}$. To this mixture was added slowly a solution of vinyl magnesium bromide in THF $(1.0 \mathrm{M}, 60 \mathrm{~mL}, 60 \mathrm{mmol}, 1.5 \mathrm{eq}$.). The reaction mixture was then stirred at $-78{ }^{\circ} \mathrm{C}$ for $5 \mathrm{~h}$ before warming up to room temperature. Saturated $\mathrm{NH}_{4} \mathrm{Cl}$ aqueous solution $(80 \mathrm{~mL})$ was introduced and the resulting mixture was stirred at room temperature for $1 \mathrm{~h}$. The mixture was diluted with water $(150 \mathrm{~mL})$ and extracted with dichloromethane $(3 \times 100 \mathrm{~mL})$ and the combined organic phases were dried over anhydrous $\mathrm{Na}_{2} \mathrm{SO}_{4}$. After filtration and concentrated, the residue was chromatographed on silica gel (petroleum ether $60-90{ }^{\circ} \mathrm{C}$ :ethyl acetate $=2: 1)$ to afford the major amide $(\mathbf{2 0 c}: 16.35 \mathrm{~g}$, $78 \%)$ as a yellow syrup. Further elution with solvents (petroleum ether $60-90{ }^{\circ} \mathrm{C}$ :ethyl acetate $=1: 2$ ) provided the minor sulfinamide $20 \mathrm{~d}(1.64 \mathrm{~g}, 7.8 \%)$ as a pale yellow oil.

\subsubsection{Major: $20 c$}

$[\alpha]_{\mathrm{D}}^{20}+20\left(\mathrm{c} 0.50, \mathrm{CHCl}_{3}\right) \cdot R_{\mathrm{f}} 0.56$ (petroleum ether:ethyl acetate $=1: 1)$. FTIR $(\mathrm{KBr}$, thin film $) \mathrm{cm}^{-1} 3223,2963$, 1640, 1462, 1397, 1269, 1199, 1061. ${ }^{1} H-N M R(400 \mathrm{MHz}$, $\left.\mathrm{CDCl}_{3}\right), \delta(\mathrm{ppm}) 7.92(1 \mathrm{H}, d, J=8.0 \mathrm{~Hz}), 7.30-7.13(6 \mathrm{H}$, $m), 7.03(1 \mathrm{H}, t, J=7.6 \mathrm{~Hz}), 6.68(1 \mathrm{H}, d, J=8.0 \mathrm{~Hz})$, $5.78(1 \mathrm{H}, d d d, J=6.4,10.8,17.2 \mathrm{~Hz}), 5.65(1 \mathrm{H}, d$, $J=14.4 \mathrm{~Hz}), 5.18(1 \mathrm{H}, d d, J=10.8,17.2 \mathrm{~Hz}), 5.09(1 \mathrm{H}$, $d, J=10.4 \mathrm{~Hz}), 3.91(1 \mathrm{H}, d, J=14.4 \mathrm{~Hz}), 3.73-3.63$ $(1 \mathrm{H}, m), 3.37(1 \mathrm{H}, t, J=8.4 \mathrm{~Hz}), 2.12-1.88(4 \mathrm{H}, m), 1.11$ (9H, s). ${ }^{13} C$-NMR (rotamer in brackets, $100 \mathrm{MHz}, \mathrm{CDCl}_{3}$ ), $\delta(\mathrm{ppm}) 171.89,143.84,140.27,139.42,136.90,130.84$ (130.81), 129.92, 129.45 (129.43), 129.29, 128.42, 127.60, 116.67 (116.56), 100.44, 58.62 (58.39), 55.97, 51.69, 31.07 (30.93), 30.68 (30.46), 22.65. EI-MS $\mathrm{m} / \mathrm{z}(\%) 524\left(\mathrm{M}^{+}\right.$, $36 \%), 467$ (100), 419 (6), 404 (47), 351 (14), 309 (77), 278 (12), 238 (13), 224 (17), 182 (21), 180 (30), 146 (5), 140 (9), 110 (8), 91 (88), 77 (9). HRMS m/z Found 524.1001, Calcd. for $\mathrm{C}_{23} \mathrm{H}_{29} \mathrm{~N}_{2} \mathrm{O}_{2}$ SI $[\mathrm{M}]^{+} 524.0995$. 


\subsubsection{Minor: 20d}

$[\alpha]_{\mathrm{D}}^{20}+41\left(\mathrm{c} 0.20, \mathrm{CHCl}_{3}\right) \cdot R_{\mathrm{f}} 0.53$ (petroleum ether:ethyl acetate $=1: 1) .{ }^{1} H-N M R\left(300 \mathrm{MHz}, \mathrm{CDCl}_{3}\right), \delta(\mathrm{ppm})$ 7.37-7.13 $(7 \mathrm{H}, m), 6.99-6.90(2 \mathrm{H}, m), 5.76(1 \mathrm{H}, d d d$, $J=6.0,9.0,18.0 \mathrm{~Hz}), 5.17(1 \mathrm{H}, d, J=18.0 \mathrm{~Hz}), 5.08$ $(1 \mathrm{H}, d, J=9.0 \mathrm{~Hz}), 4.87(2 \mathrm{H}, s), 3.66(1 \mathrm{H}, d t, J=6.0$, $15.0 \mathrm{~Hz}), 3.50(1 \mathrm{H}, d, J=9.0 \mathrm{~Hz}), 2.20-2.05(2 \mathrm{H}, m)$, 1.96-1.84 (2H, m), $1.11(9 \mathrm{H}, s) .{ }^{13} C-N M R(75 \mathrm{MHz}$, $\left.\mathrm{CDCl}_{3}\right), \delta(\mathrm{ppm}) 172.35,142.19,139.47,137.42,129.68$, $128.91,128.43,128.14,127.46,116.62,58.79,56.07$, $53.13,30.92,30.75,22.65$. EI-MS $\mathrm{m} / z$ (\%) $525\left(\mathrm{M}^{+}+1\right.$, $10 \%), 511$ (5), 467 (30), 404 (10), 341 (100), 309 (21), 278 (37), 250 (4), 238 (6), 225 (36), 183 (79), 180 (15), 140 (11), 112 (6), 110 (9), 91 (78), 77 (12). HRMS m/z. Found 524.0996, Calcd. for $\mathrm{C}_{23} \mathrm{H}_{29} \mathrm{~N}_{2} \mathrm{O}_{2} \mathrm{SI}[\mathrm{M}]^{+}$524.0995.

\subsection{Synthesis of Compound 12c}

To a mixture of sodium hydride $60 \%$ in mineral oil, $1.87 \mathrm{~g}, 46.7 \mathrm{mmol}, 1.5$ eq., freshly washed with anhydrous hexane three times under nitrogen) in anhydrous THF $(50 \mathrm{~mL})$ at $0{ }^{\circ} \mathrm{C}$ was added a solution of sulfonamide 20c $(16.3 \mathrm{~g}, 31.1 \mathrm{mmol})$ in THF $(150 \mathrm{~mL})$ via syringe. After stirring at $0{ }^{\circ} \mathrm{C}$ for $10 \mathrm{~min}$, benzyl bromide $(5.5 \mathrm{~mL}$, $46.7 \mathrm{mmol}, 1.5 \mathrm{eq}$.) was added. The resulting mixture was then stirred at $0{ }^{\circ} \mathrm{C}$ for $2 \mathrm{~h}$, then at room temperature for $12 \mathrm{~h}$ under nitrogen. A powder of $\mathrm{NH}_{4} \mathrm{Cl}(2.7 \mathrm{~g}$, $50.0 \mathrm{mmol}$ ) was added and the mixture was stirred for $10 \mathrm{~min}$. After concentrated under reduced pressure, the residue was diluted with water $(150 \mathrm{~mL})$ and extracted with ethyl acetate $(3 \times 100 \mathrm{~mL})$. The combined organic phases were washed with brine $(50 \mathrm{~mL})$ and dried over anhydrous $\mathrm{Na}_{2} \mathrm{SO}_{4}$. After filtration, the solvent was removed under reduced pressure and the residue was chromatographed on silica gel (petroleum ether 60-90 ${ }^{\circ} \mathrm{C}$ :ethyl acetate $\left.=2: 1\right)$ to afford the product $(\mathbf{1 1 b}$ : $18.1 \mathrm{~g}, 95 \%)$ as a pale yellow oil.

$[\alpha]_{\mathrm{D}}^{20}-37\left(\mathrm{c} 0.12, \mathrm{CHCl}_{3}\right) . R_{\mathrm{f}} 0.54$ (petroleum ether:ethyl acetate $=2: 1) .{ }^{1} H$-NMR (as a mixture of rotamers, $\left.400 \mathrm{MHz}, \mathrm{CDCl}_{3}\right), \delta(\mathrm{ppm}) 7.90(1 \mathrm{H}, d, J=8.0 \mathrm{~Hz})$, $7.34-7.06(11 \mathrm{H}, m), 7.00(1 \mathrm{H}, t, J=7.6 \mathrm{~Hz}), 6.64(0.5 \mathrm{H}$, $d d, J=1.2,7.6 \mathrm{~Hz}), 6.60(0.5 \mathrm{H}, d d, J=1.2,7.6 \mathrm{~Hz})$, $5.74-5.59(2 \mathrm{H}, m), 5.06(1 \mathrm{H}, d d, J=6.8,10.0 \mathrm{~Hz}), 4.97$ $(1 \mathrm{H}, t, J=18.0 \mathrm{~Hz}), 4.41(1 \mathrm{H}, d d, J=10.0,16.4 \mathrm{~Hz})$, $4.02(1 \mathrm{H}, d, J=16.4 \mathrm{~Hz}), 3.85(1 \mathrm{H}, t, J=14.0 \mathrm{~Hz})$, 3.59-3.38 $(1 \mathrm{H}, m), 2.35-2.17(1 \mathrm{H}, m), 2.03-1.80(3 \mathrm{H}, m)$, $1.12(9 \mathrm{H}, s) .{ }^{13} \mathrm{C}-\mathrm{NMR}$ (rotamer in brackets, $100 \mathrm{MHz}$, $\left.\mathrm{CDCl}_{3}\right), \delta(\mathrm{ppm}) 171.61$ (171.44), 143.81, 140.21 (140.19), 138.64 (138.54), 137.54, 137.08 (137.02), 131.00, 130.87, $129.83,129.38,129.35,129.21,128.44$ (128.39), 128.11 (128.08), $127.51, \quad 127.00, \quad 117.75 \quad$ (117.69), 100.49
(100.45), 63.29 (63.08), 58.14 (58.09), 51.56, 46.27, 31.72 (31.59), 26.94 (26.88), 23.57. EI-MS m/z (\%) $614\left(\mathrm{M}^{+}\right.$, $4 \%), 509$ (7), 482 (3), 458 (1), 420 (4), 405 (5), 309 (5), 278 (4), 236 (2), 182 (8), 180 (13), 146 (7), 128 (5), 106 (12), 91 (100). HRMS m/z Found 614.1480, Calcd. for $\mathrm{C}_{30} \mathrm{H}_{35} \mathrm{~N}_{2} \mathrm{O}_{2} \mathrm{SI}[\mathrm{M}]^{+} 614.1464$.

\subsection{Synthesis of Compound 13a $+\mathbf{1 3 b}$}

A mixture of copper iodide (CuI, $133.3 \mathrm{mg}, 0.7 \mathrm{mmol}$, 0.1 eq.) and bromoanilide $\mathbf{1 2 b}(3.78 \mathrm{~g}, 7.0 \mathrm{mmol})$ in anhydrous toluene $(140 \mathrm{~mL})$ was degassed and purged with argon (three times). A solution of lithium bis(trimethylsilyl)amide (1.0 M in THF, $14 \mathrm{~mL}, 14 \mathrm{mmol}, 2.0$ eq.) was added and the resulting mixture was stirred at $80{ }^{\circ} \mathrm{C}$ (oil bath) under argon for $5 \mathrm{~h}$. After cooling to room temperature then to $0{ }^{\circ} \mathrm{C}$, a solution of anhydrous $t$-BuOOH (degassed and purged with argon, $\sim 3.0 \mathrm{M}$ in toluene, $3.5 \mathrm{~mL}$, $10.5 \mathrm{mmol}, 1.5$ eq.) was added. The reaction mixture was allowed to stir at $0{ }^{\circ} \mathrm{C}$ under argon for $3 \mathrm{~h}$. Saturated aqueous solution of $\mathrm{NH}_{4} \mathrm{Cl}(8 \mathrm{~mL})$ was added. After $30 \mathrm{~min}$, the mixture was diluted with water $(100 \mathrm{~mL})$. The aqueous phase was extracted with ethyl acetate $(3 \times 60 \mathrm{~mL})$. The combined organic phases were dried over anhydrous $\mathrm{Na}_{2} \mathrm{SO}_{4}$. After removal of the solvents, the residue was chromatographed on silica gel (petroleum ether $60-90{ }^{\circ} \mathrm{C}$ :ethyl acetate $\left.=2: 1 \rightarrow 1: 1 \rightarrow 1: 2\right)$ to afford the major product $(\mathbf{1 3 a}+\mathbf{1 3 b}$, as a mixture of $\mathrm{C} 3-\mathrm{C} 3 \mathrm{a}$ diastereomers, $2.51 \mathrm{~g}, 78.1 \%$ ) as pale yellow syrup. Further elution afforded the minor product $(13 \mathrm{c}$ : meso-isomer, $0.23 \mathrm{~g}, 7.2 \%$ ) as a pale yellow oil, which was characterized after removal of the tert-butylsulfinyl group $(\mathbf{1 3 d})$. *The anhydrous tert-butylhydroperoxide $(t-\mathrm{BuOOH})$ in toluene (ca. $\sim 3.0 \mathrm{M}$ ) was prepared by the following procedure: $70 \%$ aqueous solution of $t-\mathrm{BuOOH}(40.6 \mathrm{~mL}$, density $=0.93 \mathrm{~g} / \mathrm{mL})$ was added to toluene $(46 \mathrm{~mL})$ and the resulting water (ca. $10 \mathrm{~mL}$ ) was separated and back-extracted with toluene $(2 \times 10 \mathrm{~mL})$. The combined organic phases were then dried over anhydrous sodium sulfate. After filtration, the resulting solution was kept with $4 \AA$ molecular sieve and could be used for this reaction without further purification.

\subsubsection{3a $+13 b$}

An 83:17 mixture of diastereomers at $\mathrm{C} 3-\mathrm{C} 3^{\prime}$ position: $R_{\mathrm{f}}$ 0.45 (petroleum ether:ethyl acetate $=1: 1)$. FTIR $(\mathrm{KBr}$, thin film) $\mathrm{cm}^{-1} 3396,3057,2957,1705,1609,1460,1360$, 1176, 1072, 982, 929, 746, 703. ${ }^{1} H-N M R \quad(400 \mathrm{MHz}$, $\mathrm{CDCl} 3$, major $\mathrm{C} 3 S-\mathrm{C} 3{ }^{\prime} S$-isomer reported), $\delta$ (ppm) 7.34-7.18 $(2 \times 8 \mathrm{H}, m), 7.11-7.03(2 \times 2 \mathrm{H}, m), 6.81$ $(2 \times 1 \mathrm{H}, t, J=7.6 \mathrm{~Hz}), 6.68(2 \times 1 \mathrm{H}, d, J=7.2 \mathrm{~Hz})$, 
$6.49(2 \times 1 \mathrm{H}, \quad t, \quad J=7.6 \mathrm{~Hz}), \quad 6.27 \quad(2 \times 1 \mathrm{H}, \quad d$, $J=7.6 \mathrm{~Hz}), \quad 4.92 \quad(2 \times 1 \mathrm{H}, \quad d, \quad J=15.6 \mathrm{~Hz}), \quad 4.36$ $(2 \times 1 \mathrm{H}, d, J=15.6 \mathrm{~Hz}), 4.21(2 \times 2 \mathrm{H}, s), 3.10(2 \times 1 \mathrm{H}$, $d d d, J=4.8,12.4,12.8 \mathrm{~Hz}), 2.51(2 \times 1 \mathrm{H}, d d d, J=3.6$, $12.4, \quad 12.8 \mathrm{~Hz}), 2.37(2 \times 1 \mathrm{H}, \quad d d d, \quad J=4.0, \quad 13.2$, 13.6 Hz), 2.21-2.11 $(2 \times 1 \mathrm{H}, m), 1.17(2 \times 9 \mathrm{H}, s) .{ }^{13} \mathrm{C}$ NMR (100 MHz, $\mathrm{CDCl}_{3}$, major C3S-C3'S-isomer reported), $\delta$ (ppm) 176.69, 142.50, 137.07, 135.38, 129.02, $128.87,128.54,128.39,127.75,127.69,127.50,126.79$, 123.58, 122.17, 108.69, 58.37, 54.28, 52.40, 44.02, 43.58, 27.78, 23.45. EI-MS m/z (\%) $919\left(\mathrm{M}^{+}, 1 \%\right), 862(5), 813$ (3), 756 (50), 709 (12), 662 (25), 647 (24), 629 (12), 601 (16), 575 (49), 551 (51), 537 (39), 523 (56), 404 (18), 354 (28), 313 (22), 261 (18), 236 (25), 195 (17), 118 (27), 106 (27), 91 (100), 65 (18). HRMS m/z. Found 941.4114, Calcd. for $\mathrm{C}_{56} \mathrm{H}_{62} \mathrm{~N}_{4} \mathrm{O}_{4} \mathrm{NaS}_{2}[\mathrm{M}+\mathrm{Na}]^{+}$941.4110.

\section{$3.14 .213 c \rightarrow 13 d$}

Meso-isomer: sulfinamide (13c: $257 \mathrm{mg}, 0.28 \mathrm{mmol})$ was dissolved in methanol $(6 \mathrm{~mL})$. To this mixture was added an aqueous solution of $\mathrm{HCl}(4 \mathrm{~N}, 0.21 \mathrm{~mL}, 0.84 \mathrm{mmol}$, 3 eq.). The resulting mixture was allowed to stir at room temperature under nitrogen for $1 \mathrm{~h}$. The reaction mixture was then treated with saturated aqueous solution of sodium bicarbonate $(\sim 3 \mathrm{~mL})$ and concentrated under reduced pressure. The mixture was diluted with water $(10 \mathrm{~mL})$ and extracted with dichloromethane $(3 \times 5 \mathrm{~mL})$, the combined organic phases were dried over anhydrous $\mathrm{Na}_{2} \mathrm{SO}_{4}$. After removal of the solvent, the residue was chromatographed on silica gel (dichloromethane:methanol $=20: 1$ ) to afford the amine (13d) (175 $\mathrm{mg}, 88 \%$ ) as pale yellow syrup.

\section{$3.14 .313 d$}

$R_{\mathrm{f}} 0.35\left(\mathrm{CH}_{2} \mathrm{Cl}_{2}: \mathrm{MeOH}: \mathrm{Et}_{3} \mathrm{~N}=20: 1: 0.01\right)$. FTIR $(\mathrm{KBr}$, thin film) $\mathrm{cm}^{-1} 3475,2921,1704,1614,1454,1362,1102$, 745. ${ }^{1} \mathrm{H}-\mathrm{NMR}$ (as a mixture of rotamers, $400 \mathrm{MHz}$, $\left.\mathrm{CDCl}_{3}\right), \quad \delta(\mathrm{ppm})$ 7.32-6.29 $(2 \times 14 \mathrm{H}, m), 5.00-4.89$ $(2 \times 1 \mathrm{H}, m), 4.76(2 \times 0.17 \mathrm{H}, d, J=16.0 \mathrm{~Hz}), 4.72$ $(2 \times 0.17 \mathrm{H}, \quad d, \quad J=16.0 \mathrm{~Hz}), \quad 4.43 \quad(2 \times 0.17 \mathrm{H}, \quad d$, $J=15.2 \mathrm{~Hz}), 4.33(2 \times 0.34 \mathrm{H}, d, J=15.2 \mathrm{~Hz}), 4.19$ $(2 \times 0.17 \mathrm{H}, \quad d, J=15.2 \mathrm{~Hz}), 3.62-3.45(2 \times 2 \mathrm{H}, m)$, $3.24-3.12(2 \times 0.66 \mathrm{H}, m), 2.63-2.10(2 \times 3.34 \mathrm{H}, m), 1.18$ $(2 \times 1 \mathrm{H}$, brs $) .{ }^{13} \mathrm{C}-\mathrm{NMR}$ (as a mixture of rotamers, $\left.100 \mathrm{MHz}, \mathrm{CDCl}_{3}\right), \delta(\mathrm{ppm}) 178.20$ (177.90), 143.07 (142.89), 140.82 (140.61), 140.33 (140.21), 136.12 (136.03), 135.78 (135.62), 131.83 (131.45), 128.87 (128.84), 128.65 (128.46), 128.31 (128.19), 128.15 (128.02), 127.91, 127.86 (127.72), 127.52 (127.45), 127.32, 127.00, 126.73, 124.85 (124.80), 123.92, 122.62 (122.56), 121.71 (121.59), 120.06
(119.86), 109.40, 108.50 (108.31), 107.32 (107.01), 55.19, 53.97 (53.64), 45.44, 44.19 (44.02), 29.10 (28.96). HRMS m/ $z$ Found 711.3658, Calcd. for $\mathrm{C}_{48} \mathrm{H}_{47} \mathrm{~N}_{4} \mathrm{O}_{2}[\mathrm{M}+\mathrm{H}]^{+} 711.3699$.

\subsection{Synthesis of Compound 19}

A mixture of tris(dibenzylideneacetone)dipalladium $\left[\mathrm{Pd}_{2}(\mathrm{dba})_{3}, \mathrm{FW} 915.72,46.0 \mathrm{mg}, 0.05 \mathrm{mmol}, 0.025\right.$ eq. $]$ and triphenylphosphine (FW 262.29, $52 \mathrm{mg}, 0.2 \mathrm{mmol}$, 0.1 eq.) and bromoanilide $\mathbf{1 2 b}(1.08 \mathrm{~g}, 2.0 \mathrm{mmol})$ in anhydrous toluene $(40 \mathrm{~mL})$ was degassed and purged with argon (three times). A solution of lithium bis(trimethylsilyl)amide (1.0 M in THF, $4 \mathrm{~mL}, 4 \mathrm{mmol}, 2.0$ eq.) was added and the resulting mixture was stirred at $80{ }^{\circ} \mathrm{C}$ (oil bath) under argon for $6 \mathrm{~h}$. After cooling to room temperature then $0{ }^{\circ} \mathrm{C}$, a solution of anhydrous $t$-BuOOH ( $\sim 3.0 \mathrm{M}$ in toluene, $1.0 \mathrm{~mL}, 3 \mathrm{mmol}, 1.5$ eq.) was added and the reaction mixture was stirred at $0{ }^{\circ} \mathrm{C}$ (ca. $5 \mathrm{~h}$ ). Saturated aqueous solution of $\mathrm{NH}_{4} \mathrm{Cl}(0.5 \mathrm{~mL})$ was added. After $10 \mathrm{~min}$, anhydrous sodium sulfate (ca. 2-3 g) was added. The resulting mixture was then filtered and washed with ethyl acetate $(3 \times 5 \mathrm{~mL})$. After removal of the solvents, the residue was chromatographed on silica gel (petroleum ether $60-90{ }^{\circ} \mathrm{C}$ : ethyl acetate $=2: 1 \rightarrow 1: 1$ ) to afford the minor product (19b, $276 \mathrm{mg}, 28 \%)$ as white plates. Further elution afforded the major product (19, $489 \mathrm{mg}, 51 \%$ ) as a yellow solid.

\section{$3.15 .1 \quad 19$}

m.p. $125-127{ }^{\circ} \mathrm{C}$. $R_{\mathrm{f}} 0.54$ (petroleum ether:ethyl acetate $=1: 1)$. FTIR $(\mathrm{KBr}$, thin film $) \mathrm{cm}^{-1} 3321,3057,2958$, 2866, 1722, 1611, 1460, 1359, 1273, 1174, 1068, 928, 743, 704, 633, 596, 464. ${ }^{1} H-N M R$ (as a mixture of rotamers, $\left.400 \mathrm{MHz}, \mathrm{CDCl}_{3}\right), \delta(\mathrm{ppm}) 7.30-7.11(12 \mathrm{H}, m), 7.08$ $(0.5 \mathrm{H}, t, J=7.6 \mathrm{~Hz}), 7.07(0.5 \mathrm{H}, t, J=7.6 \mathrm{~Hz}), 6.92$ $(0.5 \mathrm{H}, t, J=7.6 \mathrm{~Hz}), 6.91(0.5 \mathrm{H}, t, J=7.6 \mathrm{~Hz}), 6.61$ $(0.5 \mathrm{H}, d, J=7.6 \mathrm{~Hz}), 6.60(0.5 \mathrm{H}, d, J=7.6 \mathrm{~Hz}), 5.07$ $(0.5 \mathrm{H}, s), 5.04(0.5 \mathrm{H}, s), 4.86(0.5 \mathrm{H}, d, J=15.6 \mathrm{~Hz}), 4.83$ $(0.5 \mathrm{H}, d, J=15.6 \mathrm{~Hz}), 4.61(0.5 \mathrm{H}, d, J=15.6 \mathrm{~Hz}), 4.59$ $(0.5 \mathrm{H}, d, J=15.6 \mathrm{~Hz}), 4.19(0.5 \mathrm{H}, d, J=15.2 \mathrm{~Hz}), 4.17$ $(0.5 \mathrm{H}, d, J=15.2 \mathrm{~Hz}), 4.02(0.5 \mathrm{H}, d, J=15.2 \mathrm{~Hz}), 4.01$ $(0.5 \mathrm{H}, d, J=15.2 \mathrm{~Hz}), 3.10-2.95(1 \mathrm{H}, m), 2.93-2.70(1 \mathrm{H}$, $m), 2.38-2.18(2 \mathrm{H}, m), 1.10(4.5 \mathrm{H}, s), 1.09(4.5 \mathrm{H}, s)$.

${ }^{13} \mathrm{C}$-NMR (rotamer in brackets, $\left.100 \mathrm{MHz}, \mathrm{CDCl}_{3}\right), \delta(\mathrm{ppm})$ 177.54, 141.86, 136.62 (136.56), 135.29, 129.86 (129.80), $129.21,128.60,128.36$ (128.30), 127.41 (127.20), 127.03 (127.00), 123.73 (123.68), 122.88, 109.24, 74.75 (74.71), 57.97, 51.24, 43.46, 42.72, 36.55 (36.43), 23.09. HRMS m/ $z$ Found 499.2028, Calcd. for $\mathrm{C}_{28} \mathrm{H}_{32} \mathrm{~N}_{2} \mathrm{NaO}_{3} \mathrm{~S}[\mathrm{M}+\mathrm{Na}]^{+}$ 499.2031. 


\section{$3.15 .219 b$}

m.p. $113-115^{\circ} \mathrm{C} . R_{\mathrm{f}} 0.52$ (petroleum ether:ethyl acetate $=2: 1)$. FTIR $(\mathrm{KBr}$, thin film $) \mathrm{cm}^{-1} 3415,3059,2980$, 2930, 1718, 1612, 1462, 1360, 1310, 1172, 1124, 996, 934, $743,702,645,521 .{ }^{1} H$-NMR (as a mixture of rotamers, $\left.400 \mathrm{MHz}, \mathrm{CDCl}_{3}\right), \delta(\mathrm{ppm}) 7.39-7.10(12 \mathrm{H}, m), 6.95(1 \mathrm{H}$, $t, J=7.6 \mathrm{~Hz}), 6.63(1 \mathrm{H}, t, J=7.6 \mathrm{~Hz}), 4.90(0.4 \mathrm{H}, d$, $J=16.0 \mathrm{~Hz}), 4.89(0.6 \mathrm{H}, d, J=16.0 \mathrm{~Hz}), 4.62(0.4 \mathrm{H}, d$, $J=15.6 \mathrm{~Hz}), 4.61(0.6 \mathrm{H}, d, J=15.6 \mathrm{~Hz}), 4.51-4.37(1 \mathrm{H}$, brs), $4.05(0.4 \mathrm{H}, s), 3.99(0.6 \mathrm{H}, s), 3.32-3.11(2 \mathrm{H}, m)$, $2.27-2.15(2 \mathrm{H}, m), 1.40(4.5 \mathrm{H}, s), 1.39(4.5 \mathrm{H}, s) .{ }^{13} C-N M R$ $\left(100 \mathrm{MHz}, \mathrm{CDCl}_{3}\right), \delta$ (ppm) 177.44, 141.95, 136.04, $135.31,129.61,129.37,128.82,128.57,127.79,127.66$, $127.15,123.77,123.18,109.52,74.81,61.62,52.20,43.69$, 42.52, 36.79, 24.79. HRMS m/z Found 515.1974, Calcd. for $\mathrm{C}_{28} \mathrm{H}_{32} \mathrm{~N}_{2} \mathrm{NaO}_{4} \mathrm{~S}[\mathrm{M}+\mathrm{Na}]^{+} 515.1975$.

\subsection{Synthesis of Compound 21a}

To a mixture of copper iodide (CuI, $190 \mathrm{mg}, 1.0 \mathrm{mmol}$, 0.1 eq.) and $o$-iodoanilide $12 \mathrm{c}(6.14 \mathrm{~g}, 10.0 \mathrm{mmol})$ in anhydrous toluene $(200 \mathrm{~mL})$ was added a solution of lithium bis(trimethylsilyl)amide $(1.0 \mathrm{M}$ in $\mathrm{THF}, 20 \mathrm{~mL}$, $20 \mathrm{mmol}, 2.0$ eq.). The resulting mixture was degassed and purged with argon (three times). The reaction mixture was then allowed to stir at $60{ }^{\circ} \mathrm{C}$ (oil bath) under argon for $5 \mathrm{~h}$. After cooling to room temperature then to $0{ }^{\circ} \mathrm{C}$, a solution of anhydrous $t$ - $\mathrm{BuOOH}$ (degassed and purged with argon, $\sim 3.0 \mathrm{M}$ in toluene, $5.0 \mathrm{~mL}, 15 \mathrm{mmol}, 1.5 \mathrm{eq}$.) was added. The reaction mixture was then stirred at $0{ }^{\circ} \mathrm{C}$ under argon for $3 \mathrm{~h}$. Saturated aqueous solution of $\mathrm{Na}_{2} \mathrm{~S}_{2} \mathrm{O}_{3}(10 \mathrm{~mL})$ was added followed by saturated aqueous solution of $\mathrm{NH}_{4} \mathrm{Cl}(10 \mathrm{~mL})$. After $30 \mathrm{~min}$, the resulting mixture was diluted with water $(200 \mathrm{~mL})$ and extracted with ethyl acetate $(3 \times 100 \mathrm{~mL})$. The combined organic phases were dried over anhydrous $\mathrm{Na}_{2} \mathrm{SO}_{4}$. After removal of the solvents, the residue was chromatographed on silica gel (petroleum ether $60-90{ }^{\circ} \mathrm{C}$ :ethyl acetate $=2: 1 \rightarrow 1: 1 \rightarrow$ 1:2) to afford the major product (21a, $2.42 \mathrm{~g}, 50 \%)$ as a pale yellow syrup. Further elution afforded the meso-isomer (21c, characterized after removal of tert-butylsulfinyl group, $0.205 \mathrm{~g}, 4.2 \%)$, followed by minor product $(\mathbf{2 1 b}$, characterized after removal of the tert-butylsulfinyl group, $0.40 \mathrm{~g}, 8.2 \%)$ as a pale yellow oil.

\subsubsection{1a (Major Isomer)}

$[\alpha]_{\mathrm{D}}^{20}-211\left(\mathrm{c} 0.14, \mathrm{CHCl}_{3}\right) \cdot R_{\mathrm{f}} 0.55$ (petroleum ether:ethyl acetate $=1: 1)$. FTIR $\left(\mathrm{KBr}\right.$, thin film) $\mathrm{cm}^{-1} 3440,2960$, $1702,1609,1465,1361,1177,1071,924,745,701 .{ }^{1} H-$ NMR $\left(400 \mathrm{MHz}, \mathrm{CDCl}_{3}\right), \delta(\mathrm{ppm}) 7.45(2 \times 2 \mathrm{H}, d$,
$J=8.0 \mathrm{~Hz}), 7.42(2 \times 2 \mathrm{H}, d, J=8.0 \mathrm{~Hz}), 7.32-7.25$ $(2 \times 1 \mathrm{H}, m), 7.21-7.11(2 \times 3 \mathrm{H}, m), 7.01(2 \times 2 \mathrm{H}, d$, $J=7.2 \mathrm{~Hz}), 6.85(2 \times 1 \mathrm{H}, d, J=7.6 \mathrm{~Hz}), 6.79(2 \times 1 \mathrm{H}$, $t, \quad J=7.6 \mathrm{~Hz}), \quad 6.52(2 \times 1 \mathrm{H}, \quad t, \quad J=7.6 \mathrm{~Hz}), \quad 6.21$ $(2 \times 1 \mathrm{H}, d, J=8.0 \mathrm{~Hz}), 5.21(2 \times 1 \mathrm{H}, d d d, J=9.6$, $10.4, \quad 16.8 \mathrm{~Hz}), 4.80(2 \times 1 \mathrm{H}, \quad d, J=15.2 \mathrm{~Hz}), 4.58$ $(2 \times 1 \mathrm{H}, d, J=16.8 \mathrm{~Hz}), 4.14(2 \times 1 \mathrm{H}, d, J=15.2 \mathrm{~Hz})$, $4.13(2 \times 1 \mathrm{H}, \quad d, \quad J=10.4 \mathrm{~Hz}), \quad 3.90(2 \times 1 \mathrm{H}, \quad d$, $J=17.2 \mathrm{~Hz}), \quad 3.84(2 \times 1 \mathrm{H}, \quad d, \quad J=17.2 \mathrm{~Hz}), \quad 3.29$ $(2 \times 1 \mathrm{H}, d d, J=11.2,13.0 \mathrm{~Hz}), 3.02-2.89(2 \times 2 \mathrm{H}, m)$, $1.18(2 \times 9 \mathrm{H}, s) .{ }^{13} \mathrm{C}-\mathrm{NMR}\left(100 \mathrm{MHz}, \mathrm{CDCl}_{3}\right), \delta(\mathrm{ppm})$ $176.36,142.92,138.63,137.51,135.60,128.65,128.57$, $128.24,128.11,127.93,127.59,127.29,126.97,125.34$, $121.21,115.89,108.09,62.93,58.10,54.96,45.28,43.83$, 33.08, 23.35. HRMS m/z Found 971.4619, Calcd. for $\mathrm{C}_{60} \mathrm{H}_{67} \mathrm{~N}_{4} \mathrm{O}_{4} \mathrm{~S}_{2}[\mathrm{M}+\mathrm{H}]^{+} 971.4604$.

\subsubsection{1b $\rightarrow$ 21d (Minor-Isomer)}

Sulfinamide (21b: $400 \mathrm{mg}, 0.41 \mathrm{mmol})$ was dissolved in methanol $(6 \mathrm{~mL})$. To this mixture was added an aqueous solution of $\mathrm{HCl}(4 \mathrm{~N}, 0.31 \mathrm{~mL}, 1.23 \mathrm{mmol}, 3$ eq.). The resulting mixture was allowed to stir at room temperature under nitrogen for $1 \mathrm{~h}$. The reaction mixture was then treated with saturated aqueous solution of sodium bicarbonate $(\sim 8 \mathrm{~mL})$ and concentrated under reduced pressure. The mixture was diluted with water $(10 \mathrm{~mL})$ and extracted with dichloromethane $(3 \times 10 \mathrm{~mL})$, the combined organic phases were dried over anhydrous $\mathrm{Na}_{2} \mathrm{SO}_{4}$. After removal of the solvent, the residue was chromatographed on silica gel (petroleum ether $60-90{ }^{\circ} \mathrm{C}$ :ethyl acetate $=2: 1$ ) to afford the diamine (21d) $(290 \mathrm{mg}, 92 \%)$ as yellow oil.

\subsubsection{1d (Minor Isomer)}

$[\alpha]_{\mathrm{D}}^{20}-153\left(\mathrm{c} 0.18, \mathrm{CHCl}_{3}\right) \cdot R_{\mathrm{f}} 0.42$ (petroleum ether:ethyl acetate $=1: 1)$. FTIR $(\mathrm{KBr}$, thin film $) \mathrm{cm}^{-1} 3420,3061$, 2925, 2843, 2357, 1712, 1608, 1482, 1460, 1358, 1175, $1110,991,921,743,701 .{ }^{1} H-N M R$ (as a mixture of rotamers, $\left.400 \mathrm{MHz}, \mathrm{CDCl}_{3}\right), \delta(\mathrm{ppm}) 7.50-6.31(2 \times 14 \mathrm{H}, m)$, 5.62-5.46 $(2 \times 1 \mathrm{H}, m), 5.10-4.72(2 \times 2.35 \mathrm{H}, m), 4.56$ $(2 \times 0.33 \mathrm{H}, \quad d, \quad J=16.0 \mathrm{~Hz}), \quad 4.44 \quad(2 \times 0.33 \mathrm{H}, \quad d$, $J=15.6 \mathrm{~Hz}), 4.33(2 \times 0.67 \mathrm{H}, d d, J=11.6,15.6 \mathrm{~Hz})$, $4.03(2 \times 0.33 \mathrm{H}, d, J=15.6 \mathrm{~Hz}), 3.66(2 \times 0.33 \mathrm{H}, d$, $J=13.2 \mathrm{~Hz}), 3.55(2 \times 0.33 \mathrm{H}, d, J=13.2 \mathrm{~Hz}), 3.49$ $(2 \times 0.33 \mathrm{H}, d, J=12.8 \mathrm{~Hz}), 3.43-3.31(2 \times 0.67 \mathrm{H}, m)$, $3.14(2 \times 0.33 \mathrm{H}, d, J=13.2 \mathrm{~Hz}), 3.02(2 \times 0.33 \mathrm{H}, d$, $J=13.2 \mathrm{~Hz}), \quad 2.91-2.80 \quad(2 \times 0.33 \mathrm{H}, \quad m), \quad 2.77$ $(2 \times 0.33 \mathrm{H}, \quad d, \quad J=12.8 \mathrm{~Hz}), 2.67-2.50(2 \times 1 \mathrm{H}, m)$, $2.50-2.38(2 \times 0.67 \mathrm{H}, m), 2.22-2.14(2 \times 0.33 \mathrm{H}, m), 0.97$ $(2 \times 1 \mathrm{H}$, brs $) .{ }^{13} \mathrm{C}-\mathrm{NMR}$ (rotamer in brackets, $100 \mathrm{MHz}$, $\left.\mathrm{CDCl}_{3}\right), \delta$ (ppm) 179.54 (179.21), 143.82 (143.70), 141.22, 
140.53 (140.42), 140.35, 140.14 (140.09), 136.39, 136.06 (135.86), $130.91,128.87$ (128.61), 128.49 (128.45), 128.36, 128.21 (128.13), 128.04, 127.86 (127.83), 127.52, 127.39 (127.34), 127.24, 126.76 (126.59), 126.42 (126.35), $125.26,124.76,121.94$ (121.29), 119.45, 115.73, 115.44 (115.21), 109.33, 108.16 (107.18), 58.37, 58.09 (57.68), 54.81, 54.74 (54.59), 50.81, 50.75 (50.42), 44.57 (44.42), 44.11 (44.06), 34.89. HRMS $\mathrm{m} / \mathrm{z}$ Found 763.4008, Calcd. for $\mathrm{C}_{52} \mathrm{H}_{51} \mathrm{~N}_{4} \mathrm{O}_{2}[\mathrm{M}+\mathrm{H}]^{+}$: 763.4012 .

\subsubsection{1c $\rightarrow$ 21e (Meso-isomer)}

Sulfinamide (21c: $205 \mathrm{mg}, 0.21 \mathrm{mmol}$ ) was dissolved in methanol $(6 \mathrm{~mL})$. To this mixture was added an aqueous solution of $\mathrm{HCl}(4 \mathrm{~N}, 0.16 \mathrm{~mL}, 0.63 \mathrm{mmol}, 3$ eq.). The resulting mixture was allowed to stir at room temperature under nitrogen for $1 \mathrm{~h}$. The reaction mixture was then treated with saturated aqueous solution of sodium bicarbonate $(\sim 4 \mathrm{~mL})$ and concentrated under reduced pressure. The mixture was diluted with water $(10 \mathrm{~mL})$ and extracted with dichloromethane $(3 \times 5 \mathrm{~mL})$, the combined organic phases were dried over anhydrous $\mathrm{Na}_{2} \mathrm{SO}_{4}$. After removal of the solvent, the residue was chromatographed on silica gel (petroleum ether $60-90{ }^{\circ} \mathrm{C}$ :ethyl acetate $=1: 2$ ) to afford the diamine (21e) (145 $\mathrm{mg}, 91 \%)$ as yellow syrup.

\subsubsection{1e (Meso-isomer)}

$[\alpha]_{\mathrm{D}}^{20}-29\left(\mathrm{c} 0.18, \mathrm{CHCl}_{3}\right) . R_{\mathrm{f}} 0.41$ (petroleum ether:ethyl acetate $=1: 3)$. FTIR $(\mathrm{KBr}$, thin film $) \mathrm{cm}^{-1} 3311,3062$, 2975, 2924, 2846, 1704, 1613, 1492, 1455, 1354, 1257, $1182,1113,995,921,742,703,634 .{ }^{1} H-N M R$ (as a mixture of rotamers, $400 \mathrm{MHz}, \mathrm{CDCl}_{3}$ ), $\delta$ (ppm) 7.23-7.01 $(2 \times 9 \mathrm{H}, m), 6.96-6.60(2 \times 4 \mathrm{H}, m), 6.48(2 \times 0.5 \mathrm{H}, d$, $J=8.0 \mathrm{~Hz}), 6.45(2 \times 0.5 \mathrm{H}, d, J=8.0 \mathrm{~Hz}), 5.56-5.35$ $(2 \times 1 \mathrm{H}, \quad m), 4.98(2 \times 0.5 \mathrm{H}, d, J=16.0 \mathrm{~Hz}), 4.87$ $(2 \times 0.5 \mathrm{H}, \quad d, \quad J=10.0 \mathrm{~Hz}), \quad 4.87 \quad(2 \times 0.5 \mathrm{H}, \quad d$, $J=10.0 \mathrm{~Hz}), 4.76(2 \times 0.5 \mathrm{H}, d, J=15.6 \mathrm{~Hz}), 4.68$ $(2 \times 0.5 \mathrm{H}, \quad d, \quad J=16.8 \mathrm{~Hz}), \quad 4.54 \quad(2 \times 0.5 \mathrm{H}, \quad d$, $J=16.8 \mathrm{~Hz}), 4.32(2 \times 0.5 \mathrm{H}$, brs $), 3.88(2 \times 0.5 \mathrm{H}$, brs $)$, $3.55(2 \times 0.5 \mathrm{H}, d, J=13.2 \mathrm{~Hz}), 3.49(2 \times 0.5 \mathrm{H}, d$, $J=13.2 \mathrm{~Hz}), 3.13(2 \times 0.5 \mathrm{H}, d, J=13.2 \mathrm{~Hz}), 3.01$ $(2 \times 0.5 \mathrm{H}, d, J=13.2 \mathrm{~Hz}), 3.00(2 \times 0.5 \mathrm{H}$, brs $), 2.92$ $(2 \times 1 \mathrm{H}, b r s), 2.68-2.50(2 \times 2 \mathrm{H}, m), 0.97(2 \times 1 \mathrm{H}, b r s)$. ${ }^{13} \mathrm{C}$-NMR (rotamer in brackets, $\left.100 \mathrm{MHz}, \mathrm{CDCl}_{3}\right), \delta(\mathrm{ppm})$ 178.05 (176.96), 145.05 (144.20), 140.49 (140.39), 140.31 (140.19), 136.17 (135.85), 128.56 (128.44), 128.22 $(128.00), \quad 127.55, \quad 127.25 \quad(127.21), \quad 126.95, \quad 126.69$ (126.56), $\quad 125.10, \quad 124.54, \quad 121.74 \quad(121.45), \quad 115.99$ (115.44), 109.41 (109.30), 58.75 (58.56), 55.83 (55.34), 50.92 (50.85), 44.37, 37.69 (36.62). HRMS m/z Found 763.4003, Calcd. for $\mathrm{C}_{52} \mathrm{H}_{51} \mathrm{~N}_{4} \mathrm{O}_{2}[\mathrm{M}+\mathrm{H}]^{+} 763.4012$.
3.17 Synthesis of Compound 22

A solution of $21 \mathrm{a}$ (485 $\mathrm{mg}, 0.5 \mathrm{mmol}$ ) in dichloromethane and methanol $\left(20 \mathrm{~mL}, 1: 1\right.$ mixture) was cooled to $-78^{\circ} \mathrm{C}$ (dry ice-acetone bath). Ozone was then passed through the solution for $10 \mathrm{~min}$. The reaction progress was monitored by TLC. Sodium borohydride (189 mg, $5 \mathrm{mmol}, 10$ eq.) was added. The reaction mixture was then gradually warmed up to room temperature under argon at stirration overnight. Saturated aqueous solution of $\mathrm{NH}_{4} \mathrm{Cl}(10 \mathrm{~mL})$ was added. The resulting mixture was diluted with water $(20 \mathrm{~mL})$ and extracted with dichloromethane $(3 \times 20 \mathrm{~mL})$. The combined organic phases were dried over anhydrous $\mathrm{Na}_{2} \mathrm{SO}_{4}$. After removal of the solvents, the residue was chromatographed on silica gel (petroleum ether 60$90{ }^{\circ} \mathrm{C}$ :ethyl acetate $\left.=1: 2\right)$ to afford the product (22, $401 \mathrm{mg}, 82 \%$ ) as a pale yellow syrup.

$[\alpha]_{\mathrm{D}}^{20}-332\left(\mathrm{c} 0.14, \mathrm{CHCl}_{3}\right) . R_{\mathrm{f}} 0.45$ (petroleum ether:ethyl acetate $=1: 2)$. FTIR $\left(\mathrm{KBr}\right.$, thin film) $\mathrm{cm}^{-1} 3730$, 3436, 2921, 2351, 1703, 1609, 1460, 1364, 1174, 1046, 745. ${ }^{1} \mathrm{H}-\mathrm{NMR}\left(400 \mathrm{MHz}, \mathrm{CDCl}_{3}\right), \delta(\mathrm{ppm}) 7.51(2 \times 2 \mathrm{H}$, $d, J=7.6 \mathrm{~Hz}), 7.35(2 \times 2 \mathrm{H}, t, J=7.6 \mathrm{~Hz}), 7.25-7.16$ $(2 \times 4 \mathrm{H}, m), 7.04(2 \times 2 \mathrm{H}, d d, J=1.6,7.6 \mathrm{~Hz}), 6.92$ $(2 \times 1 \mathrm{H}, d, J=6.8 \mathrm{~Hz}), 6.88(2 \times 1 \mathrm{H}, d, J=8.0 \mathrm{~Hz})$, $6.63(2 \times 1 \mathrm{H}, \quad t, \quad J=7.6 \mathrm{~Hz}), \quad 6.32(2 \times 1 \mathrm{H}, \quad d$, $J=8.0 \mathrm{~Hz}), 4.84(2 \times 1 \mathrm{H}, d, J=15.6 \mathrm{~Hz}), 4.52(2 \times 1 \mathrm{H}$, $d, J=16.8 \mathrm{~Hz}), 4.35(2 \times 1 \mathrm{H}, d, J=15.6 \mathrm{~Hz}), 3.90$ $(2 \times 1 \mathrm{H}, d, J=16.8 \mathrm{~Hz}), 3.12(2 \times 1 \mathrm{H}, d d d, J=3.6$, $11.0,12.8 \mathrm{~Hz}), 3.03(2 \times 1 \mathrm{H}, d d, J=9.2,14.0 \mathrm{~Hz}), 2.92$ $(2 \times 1 \mathrm{H}, d, J=14.0 \mathrm{~Hz}), 2.59(2 \times 1 \mathrm{H}, d d d, J=3.2,9.2$, $12.8 \mathrm{~Hz}), 2.05-1.97(2 \times 1 \mathrm{H}, m), 1.20(2 \times 9 \mathrm{H}, s) .{ }^{13} \mathrm{C}-$ NMR (100 MHz, $\mathrm{CDCl}_{3}$ ), $\delta$ (ppm) 176.31, 142.80, 138.33, 135.12, 129.09, 128.92, 128.78, 128.38, 127.78, 127.63, $127.35,124.16,122.28,109.11,63.92,63.28,58.37,55.42$, 45.36, 44.02, 30.25, 23.75. HRMS $\mathrm{m} / \mathrm{z}$ Found 979.4513, Calcd. for $\mathrm{C}_{58} \mathrm{H}_{67} \mathrm{~N}_{4} \mathrm{O}_{6} \mathrm{~S}_{2}[\mathrm{M}+\mathrm{H}]^{+} 979.4502$.

\subsection{Synthesis of Compound 23}

To a mixture of diol 22 (49 $\mathrm{mg}, 0.05 \mathrm{mmol}$ ), trimethylamine (20 mg, $0.027 \mathrm{~mL}, 0.2 \mathrm{mmol})$ and DMAP (3 mg, $0.025 \mathrm{mmol})$ in dichloromethane $(5 \mathrm{~mL})$ was added 4 -fluorobenzene-1-sulfonyl chloride $(39 \mathrm{mg}, \quad 0.2 \mathrm{mmol}$, 4.0 eq.). The resulting mixture was then allowed to stir at room temperature for $6 \mathrm{~h}$. A solution of saturated aqueous solution of $\mathrm{NaHCO}_{3}(2 \mathrm{~mL})$ was added and diluted with water $(5 \mathrm{~mL})$. The mixture was extracted with dichloromethane $(3 \times 4 \mathrm{~mL})$. The combined organic phases were dried over anhydrous $\mathrm{Na}_{2} \mathrm{SO}_{4}$. After removal of the solvents, the residue was chromatographed on silica gel (petroleum ether $60-90{ }^{\circ} \mathrm{C}$ : ethyl acetate $=1: 1$ ) to afford the product $(\mathbf{2 3}, 60 \mathrm{mg}, 92 \%)$ as a plate. 
m.p. $127-129^{\circ} \mathrm{C} .[\alpha]_{\mathrm{D}}^{20}-160\left(\mathrm{c} 0.72, \mathrm{CHCl}_{3}\right) . R_{\mathrm{f}} 0.55$ (petroleum ether:ethyl acetate $=1: 1) . F T I R(\mathrm{KBr}$, thin film) $\mathrm{cm}^{-1} 3730,3456,2921,2351,1703,1459,1368$, 1016, 752. ${ }^{1} \mathrm{H}-\mathrm{NMR}\left(400 \mathrm{MHz}, \mathrm{CDCl}_{3}\right), \delta(\mathrm{ppm}) 7.46$ $(2 \times 2 \mathrm{H}, \quad d, \quad J=7.6 \mathrm{~Hz}), \quad 7.42-7.31 \quad(2 \times 4 \mathrm{H}, \quad m)$, $7.28-7.20(2 \times 2 \mathrm{H}, m), 7.16(2 \times 2 \mathrm{H}, t, J=7.6 \mathrm{~Hz})$, 6.96-6.76 $(2 \times 6 \mathrm{H}, m), 6.52(2 \times 1 \mathrm{H}, m), 6.23(2 \times 1 \mathrm{H}$, $d, J=7.2 \mathrm{~Hz}), 4.64(2 \times 1 \mathrm{H}, d, J=15.6 \mathrm{~Hz}), 4.63$ $(2 \times 1 \mathrm{H}, d, J=17.2 \mathrm{~Hz}), 4.13(2 \times 1 \mathrm{H}, d, J=15.6 \mathrm{~Hz})$, $3.83(2 \times 1 \mathrm{H}, \quad d, \quad J=17.2 \mathrm{~Hz}), \quad 3.82(2 \times 1 \mathrm{H}, \quad d$, $J=10.8 \mathrm{~Hz}), 3.20-3.01(2 \times 2 \mathrm{H}, m), 2.84(2 \times 1 \mathrm{H}, d d$, $J=2.8,10.8 \mathrm{~Hz}), 2.71-2.61(2 \times 1 \mathrm{H}, m), 1.27(2 \times 9 \mathrm{H}$, s). ${ }^{13} C-N M R\left(100 \mathrm{MHz}, \mathrm{CDCl}_{3}\right), \delta$ (ppm) 176.36, 166.80, $164.25,142.70,137.16,135.09,131.64,130.51,130.41$, $129.61,129.14,128.95,128.70,128.61,128.33,127.75$, $127.53,127.32,126.55,124.09,122.18,116.52,116.29$, 109.37, 70.58, 58.80, 58.48, 55.14, 46.39, 44.03, 30.62, 23.60. HRMS $\mathrm{m} / \mathrm{z}$ Found 1317.3994, Calcd. for $\mathrm{C}_{70} \mathrm{H}_{72} \mathrm{~N}_{4} \mathrm{O}_{10} \mathrm{~S}_{4} \mathrm{~F}_{2} \mathrm{Na}[\mathrm{M}+\mathrm{Na}]^{+}$1317.3997.

\subsection{Synthesis of Compound $\mathbf{1 3 \mathbf { a } ^ { \prime }}+\mathbf{1 3} \mathbf{b}^{\prime}$}

To a mixture of copper iodide (CuI, $190.4 \mathrm{mg}, 1.0 \mathrm{mmol}$, 0.1 eq.) and bromoanilide $\mathbf{1 2}^{\prime}(5.41 \mathrm{~g}, 10 \mathrm{mmol})$ in anhydrous toluene $(200 \mathrm{~mL})$ was added a solution of lithium bis(trimethylsilyl)amide $(1.0 \mathrm{M}$ in THF, $20 \mathrm{~mL}$, $20 \mathrm{mmol}, 2.0$ eq.). The resulting mixture was degassed and purged with argon (three times). After which, the reaction mixture was stirred at $80{ }^{\circ} \mathrm{C}$ (oil bath) under argon for $5 \mathrm{~h}$. After cooling to room temperature then to $0{ }^{\circ} \mathrm{C}$, a solution of anhydrous $t$-BuOOH $(\sim 3 \mathrm{M}$ in toluene, $5.0 \mathrm{~mL}$, $15 \mathrm{mmol}, 1.5$ eq.) was added. The reaction mixture was allowed to stir at $0{ }^{\circ} \mathrm{C}$ under argon for $3 \mathrm{~h}$. Saturated aqueous solution of $\mathrm{NH}_{4} \mathrm{Cl}(10 \mathrm{~mL})$ was added. After $30 \mathrm{~min}$, the mixture was diluted with water $(200 \mathrm{~mL})$. The resulting mixture was then extracted with ethyl acetate $(3 \times 100 \mathrm{~mL})$. The combined organic phases were dried over anhydrous $\mathrm{Na}_{2} \mathrm{SO}_{4}$. After removal of the solvents, the residue was chromatographed on silica gel (petroleum ether $\quad 60-90{ }^{\circ} \mathrm{C}$ :ethyl acetate $\left.=1: 1 \rightarrow 1: 2 \rightarrow 1: 3\right)$ to afford the major product $\left(\mathbf{1 3 a}^{\prime}+\mathbf{1 3}^{\prime}, 3.59 \mathrm{~g}, 78 \%\right)$ as a pale yellow syrup. Further elution afforded the minor

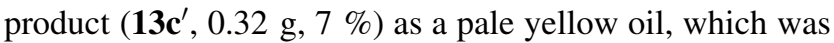
characterized after removal of tert-butylsulfinyl group (see 13d).

\section{$3.19 .113 a^{\prime}+13 b^{\prime}$}

A mixture of diastereomers at C3-C3' position (84:16): $R_{\mathrm{f}}$ 0.45 (petroleum ether:ethyl acetate $=1: 1)$. FTIR $(\mathrm{KBr}$, thin film) $\mathrm{cm}^{-1} 3429,2969,2352,1702,1612,1456,1365$, 1052. ${ }^{1} H-N M R$ (400 MHz, $\mathrm{CDCl}_{3}$, major $\mathrm{C} 3 R-\mathrm{C} 3{ }^{\prime} R$ - isomer reported), $\delta(\mathrm{ppm}) \quad 7.37-7.19(2 \times 8 \mathrm{H}, \quad m)$, $7.10-7.03(2 \times 2 \mathrm{H}, m), 6.81(2 \times 1 \mathrm{H}, t, J=7.6 \mathrm{~Hz}), 6.68$ $(2 \times 1 \mathrm{H}, d, J=7.6 \mathrm{~Hz}), 6.49(2 \times 1 \mathrm{H}, t, J=7.6 \mathrm{~Hz})$, $6.26(2 \times 1 \mathrm{H}, \quad d, \quad J=8.0 \mathrm{~Hz}), \quad 4.91 \quad(2 \times 1 \mathrm{H}, \quad d$, $J=15.6 \mathrm{~Hz}), \quad 4.36 \quad(2 \times 1 \mathrm{H}, \quad d, \quad J=15.6 \mathrm{~Hz}), \quad 4.21$ $(2 \times 2 \mathrm{H}, s), 3.11(2 \times 1 \mathrm{H}, d d d, J=4.4,12.4,12.8 \mathrm{~Hz})$, $2.48(2 \times 1 \mathrm{H}, d d d, J=3.6,12.4,12.8 \mathrm{~Hz}), 2.37(2 \times 1 \mathrm{H}$, $d d d, J=3.6,13.2,13.6 \mathrm{~Hz}), 2.22-2.10(2 \times 1 \mathrm{H}, m), 1.17$ $(2 \times 9 \mathrm{H}, s) .{ }^{13} C-N M R\left(100 \mathrm{MHz}, \mathrm{CDCl}_{3}\right.$, major $\mathrm{C} 3 R-$ $\mathrm{C}^{\prime} R$-isomer reported), $\delta(\mathrm{ppm}) 176.63,142.44,137.01$, 135.32 , 128.96, 128.81, 128.49, 128.34, 127.70, 127.64, $127.45,126.80,126.72,123.52,122.11,108.63,58.31$, $54.22,52.40,43.96,43.51,27.70,23.40$. EI-MS $\mathrm{m} / \mathrm{z}(\%)$ $919\left(\mathrm{M}^{+}, 1 \%\right), 918(1 \%), 706$ (2), 588 (3), 575 (1), 354 (3), 249 (2), 236 (4), 223 (4), 132 (10), 118 (19), 106 (7), 91 (100), 65 (5). HRMS m/z Found 918.4224, Calcd. for $\mathrm{C}_{56} \mathrm{H}_{62} \mathrm{~N}_{4} \mathrm{O}_{4} \mathrm{~S}_{2}(\mathrm{M})^{+}$918.4213; Found 919.4307, Calcd. for $\mathrm{C}_{56} \mathrm{H}_{63} \mathrm{~N}_{4} \mathrm{O}_{4} \mathrm{~S}_{2}[\mathrm{M}+\mathrm{H}]^{+} 919.4291$.

\section{Synthesis of Compound 24}

Sulfinamide $\left(\mathbf{1 3 a}^{\prime}+\mathbf{1 3}^{\prime}\right.$ : $\left.3.59 \mathrm{~g}, 3.9 \mathrm{mmol}\right)$ was dissolved in methanol $(60 \mathrm{~mL})$. To this mixture was added an aqueous solution of $\mathrm{HCl}(4 \mathrm{~N}, 2.9 \mathrm{~mL}, 11.7 \mathrm{mmol}, 3$ eq. $)$. The resulting mixture was allowed to stir at room temperature under nitrogen for $1 \mathrm{~h}$. The reaction mixture was then treated with saturated aqueous solution of sodium bicarbonate $(\sim 50 \mathrm{~mL})$ and concentrated under reduced pressure. The mixture was diluted with water $(100 \mathrm{~mL})$ and extracted with dichloromethane $(3 \times 50 \mathrm{~mL})$, the combined organic phases were dried over anhydrous $\mathrm{Na}_{2} \mathrm{SO}_{4}$. After removal of the solvent, the residue was chromatographed on silica gel (dichloromethane:methanol = 20:1) to afford the amine (24) $(2.63 \mathrm{~g}, 95 \%)$ as white foam. The diamine was dissolved in methanol $(20 \mathrm{~mL})$ and $\mathrm{HCl}(2 \mathrm{~N}, 5.6 \mathrm{~mL}, 11.1 \mathrm{mmol}, 3.0$ eq. $)$ was added. This solution was allowed to crystallize at room temperature. The needle-like crystals were collected and subjected to HPLC analysis (a 1:1 mixture of $\mathrm{C} 3 R-\mathrm{C} 3^{\prime} R$ and $\mathrm{C} 3 S-$ $\mathrm{C}^{\prime} S$ enantiomers). The mother liquid was then treated with saturated aqueous solution of sodium bicarbonate to $\mathrm{pH} 8$, and extracted with dichloromethane (3). The combined organic phases were dried over anhydrous $\mathrm{Na}_{2} \mathrm{SO}_{4}$. After removal of the solvents, the enantiomeric pure product $\mathbf{2 4}$ was obtained $(1.60 \mathrm{~g}, 61 \%)$ as a pale yellow syrup.

$[\alpha]_{\mathrm{D}}^{20}+171(\mathrm{c} \quad 0.12, \mathrm{MeOH}) . \quad e e=99.1 \% . \quad R_{\mathrm{f}} 0.40$ $\left(\mathrm{CH}_{2} \mathrm{Cl}_{2}: \mathrm{MeOH}: \mathrm{Et}_{3} \mathrm{~N}=20: 1: 0.01\right)$. FTIR $(\mathrm{KBr}$, thin film $)$ $\mathrm{cm}^{-1} 3426,2967,1701,1611,1456,1365,1174,1047$, 746, 700. ${ }^{1} \mathrm{H}-\mathrm{NMR}\left(400 \mathrm{MHz}, \mathrm{CDCl}_{3}\right), \delta$ (ppm) 7.32-7.16 $(2 \times 9 \mathrm{H}, m), 7.10(2 \times 2 \mathrm{H}, d, J=7.2 \mathrm{~Hz}), 7.04(2 \times 1 \mathrm{H}$, $d, \quad J=7.2 \mathrm{~Hz}), \quad 6.97 \quad(2 \times 1 \mathrm{H}, \quad t, \quad J=7.6 \mathrm{~Hz}), \quad 6.71$ 
$(2 \times 1 \mathrm{H}, t, J=7.6 \mathrm{~Hz}), 6.39(2 \times 1 \mathrm{H}, d, J=7.6 \mathrm{~Hz})$, $5.03(2 \times 1 \mathrm{H}, \quad d, \quad J=15.6 \mathrm{~Hz}), 4.45(2 \times 1 \mathrm{H}, \quad d$, $J=15.6 \mathrm{~Hz}), \quad 3.60(2 \times 1 \mathrm{H}, \quad d, \quad J=13.6 \mathrm{~Hz}), \quad 3.53$ $(2 \times 1 \mathrm{H}, d, J=13.6 \mathrm{~Hz}), 3.26(2 \times 1 \mathrm{H}, d d d, J=5.6$, $6.0,13.2 \mathrm{~Hz}), 2.64(2 \times 1 \mathrm{H}, d d d, J=7.6,8.0,13.2 \mathrm{~Hz})$, 2.27-2.17 $(2 \times 2 \mathrm{H}, m) .{ }^{13} \mathrm{C}-\mathrm{NMR}\left(100 \mathrm{MHz}, \mathrm{CDCl}_{3}\right), \delta$ (ppm) 178.03, 143.09, 140.31, 135.85, 128.68, 128.32, $128.06,128.01,127.85,127.58,126.74,124.12,121.76$, $108.52,55.14,53.65,45.41,44.12,29.22$. EI-MS $\mathrm{m} / \mathrm{z}(\%)$ $710\left(\mathrm{M}^{+}, 2 \%\right), 577$ (1), 356 (4), 344 (2), 262 (3), 236 (4), 223 (4), 134 (8), 118 (12), 106 (21), 91 (100). HRMS m/ $z$ Found 710.3622, Calcd. for $\mathrm{C}_{48} \mathrm{H}_{46} \mathrm{~N}_{4} \mathrm{O}_{2}[\mathrm{M}]^{+} 710.3621$.

\subsection{Synthesis of Compound 25}

To diamine $24(0.82 \mathrm{~g}, 1.15 \mathrm{mmol})$ in acetonitrile $(10 \mathrm{~mL})$ was added a solution of formaldehyde (37\% aqueous solution, $0.44 \mathrm{~mL}, 5.75 \mathrm{mmol}, 5.0$ eq.). Sodium triacetoxyborohydride (1.22 g, $5.75 \mathrm{mmol}, 5.0 \mathrm{eq}$.) was added and the resulting mixture was stirred at room temperature under argon for $2 \mathrm{~h}$. A solution of methanol in dichloromethane (MeOH: $\mathrm{CH}_{2} \mathrm{Cl}_{2}=5: 95,10 \mathrm{~mL}$ ) saturated with ammonia was added. The mixture was then allowed to stir at room temperature for $5 \mathrm{~min}$. After removal of the solvent under reduced pressure, the residue was chromatographed on silica gel (dichloromethane:MeOH: $\mathrm{NH}_{3}-$ $\left.\mathrm{H}_{2} \mathrm{O}=300: 10: 1\right)$ to afford the product $(\mathbf{2 5}, 0.82 \mathrm{~g}, 96 \%$ yield) as a pale yellow syrup.

$[\alpha]_{\mathrm{D}}^{20}+171$ (c 0.10, MeOH). $R_{\mathrm{f}} 0.45\left(\mathrm{CH}_{2} \mathrm{Cl}_{2}: \mathrm{MeOH}=\right.$ 30:1). FTIR (KBr, thin film) $\mathrm{cm}^{-1} 3436,2930,2789,1702$, 1608, 1462, 1361, 1177, 1036, 744, 700. ${ }^{1} H-N M R$ $\left(300 \mathrm{MHz}, \mathrm{CDCl}_{3}\right), \delta(\mathrm{ppm}) 7.18-7.01(2 \times 10 \mathrm{H}, m), 6.89$ $(2 \times 1 \mathrm{H}, d, J=7.5 \mathrm{~Hz}), 6.79(2 \times 1 \mathrm{H}, t, J=7.5 \mathrm{~Hz})$, $6.55(2 \times 1 \mathrm{H}, \quad t, \quad J=7.5 \mathrm{~Hz}), \quad 6.25(2 \times 1 \mathrm{H}, \quad d$, $J=7.5 \mathrm{~Hz}), \quad 4.92 \quad(2 \times 1 \mathrm{H}, \quad d, \quad J=15.0 \mathrm{~Hz}), \quad 4.32$ $(2 \times 1 \mathrm{H}, d, J=15.0 \mathrm{~Hz}), 3.26(2 \times 1 \mathrm{H}, d, J=12.9 \mathrm{~Hz})$, $3.15(2 \times 1 \mathrm{H}, d, J=12.9 \mathrm{~Hz}), 3.18-3.07(2 \times 1 \mathrm{H}, m)$, 2.67-2.53 $(2 \times 1 \mathrm{H}, m), 1.94(2 \times 3 \mathrm{H}, s), \quad 1.98-1.86$ $(2 \times 1 \mathrm{H}, m), 1.79-1.67(2 \times 1 \mathrm{H}, m) .{ }^{13} C-N M R(75 \mathrm{MHz}$, $\left.\mathrm{CDCl}_{3}\right), \delta(\mathrm{ppm}) 177.53,142.97,138.34,135.84,129.27$, $128.62,128.05,128.01,127.86,127.56,126.83,124.02$, 121.75, 108.35, 61.81, 55.00, 53.04, 43.99, 41.80, 26.11. EI-MS $m / z(\%) 738\left(\mathrm{M}^{+}, 9 \%\right), 647$ (21), 591 (43), 444 (4), 370 (23), 293 (2), 277 (13), 235 (12), 148 (17), 134 (93), 120 (25), 91 (100), 65 (10). HRMS m/z Found 738.3931, Calcd. for $\mathrm{C}_{50} \mathrm{H}_{50} \mathrm{~N}_{4} \mathrm{O}_{2}[\mathrm{M}]^{+} 738.3934$.

\subsection{Synthesis of Compound 26}

Diamine 25 (369 mg, $0.5 \mathrm{mmol}$ ) in anhydrous 1,2-dichloroethane $(15 \mathrm{~mL})$ was stirred with a solution of $\alpha$-chloroethyl chloroformate (ACE-Cl, $0.54 \mathrm{~mL}, 5 \mathrm{mmol}, 10.0$ eq.) at
$0{ }^{\circ} \mathrm{C}$ for $2 \mathrm{~h}$, then at room temperature for $1 \mathrm{~h}$. After which, the reaction mixture was allowed to stir at $80{ }^{\circ} \mathrm{C}$ (oil bath) for $12 \mathrm{~h}$. After removal of the solvents, the residue was diluted with methanol $(15 \mathrm{~mL})$ and stirred at $70{ }^{\circ} \mathrm{C}$ (oil bath) for $3 \mathrm{~h}$. The resulting mixture was concentrated under reduced pressure and diluted with dichloromethane $(5 \mathrm{~mL})$, ice $(\sim 10 \mathrm{~g})$ and saturated aqueous solution of $\mathrm{NaHCO}_{3}$ $(10 \mathrm{~mL})$. The mixture was then extracted with dichloromethane $(3 \times 15 \mathrm{~mL})$, and the combined organic phases was dried over anhydrous sodium sulfate. After filtration and removal of the solvent under reduced pressure, the residue was chromatographed on silica gel (dichloromethane:MeOH: $\left.\mathrm{NH}_{3}-\mathrm{H}_{2} \mathrm{O}=100: 100: 1\right)$ to afford the product (26, $265 \mathrm{mg}, 95 \%$ yield) as a pale yellowish syrup.

$[\alpha]_{\mathrm{D}}^{20}+203(\mathrm{c} 0.10, \mathrm{MeOH}) . R_{\mathrm{f}} 0.44\left(\mathrm{CH}_{2} \mathrm{Cl}_{2}: \mathrm{MeOH}=\right.$ 1:1). FTIR (KBr, thin film) $\mathrm{cm}^{-1} 3431,2970,2352,1628$, 1397, 1089. ${ }^{1} \mathrm{H}-\mathrm{NMR} \quad\left(400 \mathrm{MHz}, \mathrm{CDCl}_{3}\right), \delta(\mathrm{ppm})$ $7.32-7.11(2 \times 5 \mathrm{H}, m), 7.03(2 \times 1 \mathrm{H}, d, J=7.2 \mathrm{~Hz})$, $6.92(2 \times 1 \mathrm{H}, d t, J=0.8,7.6 \mathrm{~Hz}), 6.70(2 \times 1 \mathrm{H}, t$, $J=7.6 \mathrm{~Hz}), 6.35(2 \times 1 \mathrm{H}, d, J=7.6 \mathrm{~Hz}), 5.10(2 \times 1 \mathrm{H}$, $d, J=15.6 \mathrm{~Hz}), 4.40(2 \times 1 \mathrm{H}, d, J=15.6 \mathrm{~Hz}), 3.25-3.13$ $(2 \times 1 \mathrm{H}, m), 2.58-2.49(2 \times 1 \mathrm{H}, m), 2.21(2 \times 3 \mathrm{H}, s)$, 2.12-2.03 $(2 \times 3 \mathrm{H}, m) .{ }^{13} C-N M R\left(100 \mathrm{MHz}^{13} \mathrm{CDCl}_{3}\right), \delta$ (ppm) 177.73, 142.98, 135.88, 128.74, 128.20, 128.00, $127.86,127.66,124.02,121.84,108.54,55.04,47.81$, 44.05, 36.12, 29.00. EI-MS m/z (\%) $558\left(\mathrm{M}^{+}, 21 \%\right), 526$ (5), 501 (100), 470 (12), 444 (43), 280 (52), 248 (7), 236 (38), 223 (28), 187 (7), 158 (13), 91 (56). HRMS m/z Found 558.2989, Calcd. for $\mathrm{C}_{36} \mathrm{H}_{38} \mathrm{~N}_{4} \mathrm{O}_{2}[\mathrm{M}]^{+}$558.2995.

\subsection{Synthesis of Compound 27}

Diamine 26 (558 mg, $1.0 \mathrm{mmol}$ ) in THF (25 mL) was degassed and purged with argon (three times). A solution of diisobutylaluminium hydride (DIBAL-H, 1.1 M in THF, $10 \mathrm{~mL}, 10 \mathrm{mmol}, 10.0$ eq.) was added and the resulting mixture was stirred at $0{ }^{\circ} \mathrm{C}$ for $1 \mathrm{~h}$, then at room temperature for $2 \mathrm{~h}$ and finally at $80{ }^{\circ} \mathrm{C}$ (oil bath) under argon for $15 \mathrm{~h}$. After cooling to room temperature, a saturated aqueous solution of potassium sodium tartrate $(10 \mathrm{~mL})$ was added and the resulting mixture was stirred at room temperature for $2 \mathrm{~h}$. The mixture was diluted with water $(30 \mathrm{~mL})$ and extracted with ethyl acetate $(3 \times 30 \mathrm{~mL})$. The combined organic phases were dried over anhydrous sodium sulfate. After filtration and removal of the solvent under reduced pressure, the residue was chromatographed on silica gel (petroleum ether $60-90{ }^{\circ} \mathrm{C}$ : ethyl acetate $=1: 4$ ) to afford the product $(27,284 \mathrm{mg}, 54 \%$ yield) as white foam.

$[\alpha]_{\mathrm{D}}^{20}+248\left(\mathrm{c} 0.11, \mathrm{CHCl}_{3}\right) \cdot R_{\mathrm{f}} 0.54$ (petroleum ether:ethyl acetate $=1: 4)$. FTIR $(\mathrm{KBr}$, thin film $) \mathrm{cm}^{-1} 3419$, 3029, 2919, 2791, 1598, 1488, 1350, 1258, 1147, 1039, 736. ${ }^{1} \mathrm{H}-\mathrm{NMR}\left(400 \mathrm{MHz}, \mathrm{CDCl}_{3}\right), \delta(\mathrm{ppm}) 7.31-7.21(2 \times 4 \mathrm{H}$, 
$m), 7.20-7.13(2 \times 1 \mathrm{H}, m), 6.90(2 \times 1 \mathrm{H}, d, J=6.8 \mathrm{~Hz})$, $6.82(2 \times 1 \mathrm{H}, t, J=7.6 \mathrm{~Hz}), 6.46(2 \times 1 \mathrm{H}, t, J=7.6 \mathrm{~Hz})$, $6.10(2 \times 1 \mathrm{H}, d, J=7.6 \mathrm{~Hz}), 4.49(2 \times 1 \mathrm{H}, b r s), 4.42$ $(2 \times 1 \mathrm{H}, d, J=16.4 \mathrm{~Hz}), 4.36(2 \times 1 \mathrm{H}, d, J=16.4 \mathrm{~Hz})$, $2.57-2.50(2 \times 2 \mathrm{H}, m), 2.42-2.33(2 \times 1 \mathrm{H}, m), \quad 2.18$ $(2 \times 3 \mathrm{H}, s), 1.94-1.87(2 \times 1 \mathrm{H}, m) .{ }^{13} C-N M R(100 \mathrm{MHz}$, $\left.\mathrm{CDCl}_{3}\right), \delta(\mathrm{ppm}) 152.69,139.44,133.08,128.54,128.09$, $127.39,126.87,126.73,124.13,117.29,106.80,92.96$, 63.27, 53.21, 52.61, 38.99, 35.74. EI-MS $\mathrm{m} / z(\%) 526\left(\mathrm{M}^{+}\right.$, 10\%), 482 (6), 439 (26), 392 (3), 309 (12), 263 (49), 262 (100), 220 (15), 172 (26), 171 (19), 130 (8), 91 (42). HRMS $\mathrm{m} / z$ Found 526.3099, Calcd. for $\mathrm{C}_{36} \mathrm{H}_{38} \mathrm{~N}_{4}[\mathrm{M}]^{+} 526.3096$.

\subsection{Synthesis of (+)-Chimonanthine}

To a solution of liquid ammonia (freshly distilled and collected by Birch condenser, acetone-dry ice, $50-60 \mathrm{~mL}$ ) at $-78{ }^{\circ} \mathrm{C}$ was added sodium metal (ca. $124 \mathrm{mg}$, $5.4 \mathrm{mmol}, 10$ eq.). A solution of chimonanthine precursor $(27,284 \mathrm{mg}, 0.54 \mathrm{mmol})$ in anhydrous THF $(10 \mathrm{~mL})$ was added to this dark blue solution of liquid ammonia. After stirring at $-78{ }^{\circ} \mathrm{C}$ for $15 \mathrm{~min}$, a powder of $\mathrm{NH}_{4} \mathrm{Cl}$ (433 $\mathrm{mg}, 8.1 \mathrm{mmol}$ ) was added in one portion followed by saturated aqueous solution of $\mathrm{NH}_{4} \mathrm{Cl}(5 \mathrm{~mL})$. The resulting mixture was allowed to evaporate in fume hood. The residue was then diluted with water $(20 \mathrm{~mL})$ and extracted with dichloromethane $(3 \times 20 \mathrm{~mL})$. The organic phases were combined and dried over anhydrous sodium sulfate. After filtration, the solvent was removed under reduced pressure and the crude product was chromatographed on silica gel $\left(\mathrm{CH}_{2} \mathrm{Cl}_{2}: \mathrm{MeOH}: \mathrm{NH}_{3}-\mathrm{H}_{2} \mathrm{O}=200: 10: 1\right)$ to afford the product $(2,177 \mathrm{mg}, 95 \%)$ as white plates.

m.p. $170-172{ }^{\circ} \mathrm{C},[\alpha]_{\mathrm{D}}^{20}+285$ (c 0.12 , EtOH). $R_{\mathrm{f}} 0.48$ $\left(\mathrm{CH}_{2} \mathrm{Cl}_{2}: \mathrm{MeOH}: \mathrm{NH}_{3}-\mathrm{H}_{2} \mathrm{O}=200: 10: 1\right)$. FTIR $(\mathrm{KBr}$, thin film) $\mathrm{cm}^{-1} 3404,3219,2930,2856,2797,1601,1480,1252$, $1161,1031,739,648 .{ }^{1} H-N M R\left(300 \mathrm{MHz}, \mathrm{CDCl}_{3}\right), \delta(\mathrm{ppm})$ $7.19(2 \times 1 \mathrm{H}, \quad d, \quad J=7.2 \mathrm{~Hz}), \quad 6.99(2 \times 1 \mathrm{H}, \quad t$, $J=7.2 \mathrm{~Hz}), 6.67(2 \times 1 \mathrm{H}, t, J=7.2 \mathrm{~Hz}), 6.54(2 \times 1 \mathrm{H}$, $d, J=7.2 \mathrm{~Hz}), 4.34(2 \times 1 \mathrm{H}, b r s), 4.12(2 \times 1 \mathrm{H}, b r s)$, 2.63-2.48 $(2 \times 3 \mathrm{H}, m), 2.30(2 \times 3 \mathrm{H}, s), 2.18-2.01$ $(2 \times 1 \mathrm{H}, m) .{ }^{13} C-N M R\left(75 \mathrm{MHz}, \mathrm{CDCl}_{3}\right), \delta(\mathrm{ppm}) 150.74$, 133.27, 128.27, 124.58, 118.78, 109.41, 85.40, 63.41, 52.83, 37.38, 35.66. EI-MS $m / z(\%) 346\left(\mathrm{M}^{+}, 7 \%\right), 302(2), 259$ (2), 245 (3), 231 (3), 190 (11), 173 (37), 172 (100), 157 (6), 143 (8), 130 (30), 117 (6), 103 (5), 85 (24), 83 (28). HRMS $\mathrm{m} / \mathrm{z}$ Found 346.2151, Calcd. for $\mathrm{C}_{22} \mathrm{H}_{26} \mathrm{~N}_{4}[\mathrm{M}]^{+} 346.2157$.

\subsection{Synthesis of (+)-Folicanthine}

To a solution of amine $2(35 \mathrm{mg}, 0.1 \mathrm{mmol})$ in acetonitrile $(3 \mathrm{~mL})$ was added a solution of formalin $(37 \% \mathrm{HCHO}$ in water, $39 \mu \mathrm{L}, \quad 0.52 \mathrm{mmol}, \quad 5.2$ eq.) and sodium triacetoxyborohydride $\left[\mathrm{NaBH}(\mathrm{OAc})_{3}, 110 \mathrm{mg}, 0.52 \mathrm{mmol}\right]$. The resulting mixture was then stirred at room temperature under argon for $1 \mathrm{~h}$. The mixture was then treated with a solution of methanol in dichloromethane saturated with ammonia (ca. $5 \mathrm{~mL}, \mathrm{CH}_{2} \mathrm{Cl}_{2}: \mathrm{MeOH}=95: 5$ ). After stirring for $5 \mathrm{~min}$, the mixture was concentrated and the residue was chromatographed on silica gel (dichloromethane: methanol: $\left.\mathrm{NH}_{3}-\mathrm{H}_{2} \mathrm{O}=500: 10: 1\right)$ to afford the product (3, $35.5 \mathrm{mg}, 95 \%)$ as white plates.

m.p. $183-185{ }^{\circ} \mathrm{C},[\alpha]_{\mathrm{D}}^{20}+315$ (c $\left.0.10, \mathrm{MeOH}\right) . R_{\mathrm{f}} 0.48$ $\left(\mathrm{CH}_{2} \mathrm{Cl}_{2}: \mathrm{MeOH}: \mathrm{NH}_{3}-\mathrm{H}_{2} \mathrm{O}=200: 10: 1\right)$. FTIR $(\mathrm{KBr}$, thin film) $\mathrm{cm}^{-1} 3434,2944,2784,1601,1488,1345,1156,1034$, 730. ${ }^{1} \mathrm{H}-\mathrm{NMR}\left(300 \mathrm{MHz}, \mathrm{CDCl}_{3}\right), \delta$ (ppm) 7.02-6.88 $(2 \times 2 \mathrm{H}, m), 6.50(2 \times 1 \mathrm{H}, t, J=7.5 \mathrm{~Hz}), 6.26(2 \times 1 \mathrm{H}, d$, $J=7.5 \mathrm{~Hz}), 4.38(2 \times 1 \mathrm{H}$, brs $), 3.00(2 \times 3 \mathrm{H}, s), 2.70-2.58$ $(2 \times 1 \mathrm{H}, m), 2.51-2.33(2 \times 2 \mathrm{H}, m), 2.41(2 \times 3 \mathrm{H}, s)$, 2.04-1.91 $(2 \times 1 \mathrm{H}, m) .{ }^{13} C-N M R\left(75 \mathrm{MHz}, \mathrm{CDCl}_{3}\right), \delta(\mathrm{ppm})$ 153.00, 132.95, 128.14, 123.70, 116.71, 105.90, 92.03, 62.74, 52.72, 38.03, 35.52, 35.39. EI-MS $m / z(\%) 374\left(\mathrm{M}^{+}, 11 \%\right)$, 273 (2), 187 (45), 186 (100), 172 (9), 157 (8), 145 (14), 144 (31), 130 (7), 115 (5), 85 (19), 83 (22). HRMS m/z. Found 374.2480, Calcd. for $\mathrm{C}_{24} \mathrm{H}_{30} \mathrm{~N}_{4}[\mathrm{M}]^{+} 374.2470$.

\subsection{Synthesis of (-)-Calycanthine}

To a solution of acetic acid in $\mathrm{D}_{2} \mathrm{O}(0.43 \mathrm{M}, 4 \mathrm{~mL})$ was added chimonanthine 2 ( $35 \mathrm{mg}, 0.1 \mathrm{mmol})$. The resulting mixture was then stirred at $95{ }^{\circ} \mathrm{C}$ for $18 \mathrm{~h}$ under an atmosphere of argon. After cooling down to room temperature, the mixture was diluted with dichloromethane $(10 \mathrm{~mL})$ and treated with a saturated aqueous solution of sodium bicarbonate (until $\mathrm{pH}$ 8). The combined aqueous phases were back-extracted with dichloromethane $(3 \times$ $8 \mathrm{~mL}$ ). The organic phases were combined and dried over anhydrous sodium sulfate. After filtration and removal of the solvent, the residue was chromatographed on silica gel (dichloromethane:methanol: $\mathrm{NH}_{3}-\mathrm{H}_{2} \mathrm{O}=500: 10: 1$ ) to afford the product $(4,18 \mathrm{mg}, 52 \%)$ as a white solid.

m.p. $232-235^{\circ} \mathrm{C},[\alpha]_{\mathrm{D}}^{20}-615$ (c 0.15 , EtOH). FTIR (KBr, thin film) $\mathrm{cm}^{-1} 3435,2968,1627,1451,1047,744$, 608. ${ }^{l} H-N M R\left(300 \mathrm{MHz}, \mathrm{CDCl}_{3}\right), \delta(\mathrm{ppm}) 7.01(2 \times 1 \mathrm{H}$, $d, \quad J=7.5 \mathrm{~Hz}), \quad 6.82(2 \times 1 \mathrm{H}, \quad t, \quad J=7.5 \mathrm{~Hz}), \quad 6.55$ $(2 \times 1 \mathrm{H}, t, J=7.5 \mathrm{~Hz}), 6.28(2 \times 1 \mathrm{H}, d, J=7.5 \mathrm{~Hz})$, $4.69(2 \times 1 \mathrm{H}$, brs $), 4.44(2 \times 1 \mathrm{H}, s), 3.17(2 \times 1 \mathrm{H}, d d d$, $J=5.4,13.2,13.2 \mathrm{~Hz}), 2.70(2 \times 1 \mathrm{H}, d d, J=4.6$, $11.4 \mathrm{~Hz}), 2.46(2 \times 3 \mathrm{H}, s), 2.29(2 \times 1 \mathrm{H}, d d d, J=3.6$, $11.4,11.4 \mathrm{~Hz}), 1.33(2 \times 1 \mathrm{H}, d d, J=3.6,13.2 \mathrm{~Hz}) .{ }^{13} C-$ NMR $\left(75 \mathrm{MHz}, \mathrm{CDCl}_{3}\right), \delta$ (ppm) 145.00, 126.88, 124.58, 116.90, 112.36, 71.37, 46.63, 42.50, 35.96, 31.55. EI-MS $\mathrm{m} / \mathrm{z}(\%) 347\left(\mathrm{M}^{+}+\mathrm{H}, 31 \%\right), 346\left(\mathrm{M}^{+}, 100 \%\right), 314(5)$, 302 (18), 288 (29), 270 (23), 259 (13), 245 (28), 231 (59), 219 (11), 199 (9), 185 (13), 172 (21), 149 (30), 143 (27), 
130 (27), 115 (14), 87 (69), 83 (72), 74 (91). HRMS m/ $z$ Found 346.2149, Calcd. for $\mathrm{C}_{22} \mathrm{H}_{26} \mathrm{~N}_{4}[\mathrm{M}]^{+} 346.2157$.

\subsection{Synthesis of Compound 28}

To a solution of sulfinamide $21 \mathrm{a}(2.42 \mathrm{~g}, 2.5 \mathrm{mmol})$ in methanol $(50 \mathrm{~mL})$ was added an aqueous solution of $\mathrm{HCl}$ (4 N, $1.88 \mathrm{~mL}, 7.5 \mathrm{mmol}, 3$ eq.). The resulting mixture was allowed to stir at room temperature for $1 \mathrm{~h}$. The reaction mixture was then treated with saturated aqueous solution of sodium bicarbonate (until $\mathrm{pH} 8$ ) and concentrated under reduced pressure. The mixture was diluted with water $(80 \mathrm{~mL})$ and extracted with dichloromethane $(3$ $\times 50 \mathrm{~mL}$ ), the combined organic phases were dried over anhydrous $\mathrm{Na}_{2} \mathrm{SO}_{4}$. After removal of the solvent, the residue was chromatographed on silica gel (petroleum ether $60-90{ }^{\circ} \mathrm{C}$ :ethyl acetate $=2: 1$ ) to afford the amine (28) $(1.81 \mathrm{~g}, 95 \%)$ as a pale yellow syrup.

$[\alpha]_{\mathrm{D}}^{20}-241\left(\mathrm{c} 0.18, \mathrm{CHCl}_{3}\right) . e e=99.8 \%, R_{\mathrm{f}} 0.65$ (petroleum ether:ethyl acetate $=2: 1)$. FTIR $(\mathrm{KBr}$, thin film) $\mathrm{cm}^{-1} 3454,3061,2921,1699,1609,1485,1361,742 .{ }^{1} H$ NMR $\left(400 \mathrm{MHz}, \mathrm{CDCl}_{3}\right), \delta(\mathrm{ppm}) 7.23-7.15(2 \times 5 \mathrm{H}, m)$, 7.11-7.04 $(2 \times 3 \mathrm{H}, m), 6.98(2 \times 1 \mathrm{H}, t, J=6.8 \mathrm{~Hz}), 6.97$ $(2 \times 1 \mathrm{H}, d, J=7.2 \mathrm{~Hz}), 6.72-6.66(2 \times 2 \mathrm{H}, m), 6.61$ $(2 \times 1 \mathrm{H}, t, J=7.2 \mathrm{~Hz}), 6.35(2 \times 1 \mathrm{H}, t, J=8.0 \mathrm{~Hz}), 5.52$ $(2 \times 1 \mathrm{H}, d d d, J=8.4,10.0,17.2 \mathrm{~Hz}), 5.00(2 \times 1 \mathrm{H}, d$, $J=10.0 \mathrm{~Hz}), \quad 4.75 \quad(2 \times 1 \mathrm{H}, \quad d, \quad J=17.2 \mathrm{~Hz}), \quad 4.48$ $(2 \times 1 \mathrm{H}, d, J=15.6 \mathrm{~Hz}), 4.42(2 \times 1 \mathrm{H}, d, J=15.6 \mathrm{~Hz})$, $3.50(2 \times 1 \mathrm{H}, d, J=13.2 \mathrm{~Hz}), 3.33(2 \times 1 \mathrm{H}, d d, J=4.0$, $13.6 \mathrm{~Hz}), 3.01(2 \times 1 \mathrm{H}, d, J=13.2 \mathrm{~Hz}), 2.54(2 \times 1 \mathrm{H}$, $d d d, J=4.0,8.6,10.8 \mathrm{~Hz}), 2.40(2 \times 1 \mathrm{H}, d d, J=10.8$, $13.6 \mathrm{~Hz}), 0.91(2 \times 1 \mathrm{H}$, brs $) .{ }^{13} \mathrm{C}-\mathrm{NMR}\left(100 \mathrm{MHz}, \mathrm{CDCl}_{3}\right)$, $\delta(\mathrm{ppm}) 179.28,143.86,140.53,140.01,136.12,128.44$, $128.07,128.02,127.87,127.70,127.31,126.32,124.88$, 121.36, 115.53, 108.44, 57.58, 54.78, 50.31, 44.45, 34.85 . +TOF-MS $m / z(\%) 763\left(\mathrm{M}^{+}+1,100 \%\right), 382(15), 275(2)$, 236 (20). HRMS m/z Found 763.4015, Calcd. for $\mathrm{C}_{52} \mathrm{H}_{51} \mathrm{~N}_{4} \mathrm{O}_{2}[\mathrm{M}+\mathrm{H}]^{+} 763.4012$.

\subsection{Synthesis of Compound 28a}

To diamine 28 (763 mg, $1 \mathrm{mmol}$ ) in dichloromethane $(20 \mathrm{~mL})$ was added a powder of $\mathrm{N}$-chlorosuccinimide (NCS, $400 \mathrm{mg}, 3 \mathrm{mmol}, 3.0$ eq.). The resulting mixture was stirred at room temperature for $1 \mathrm{~h}$. After which, a solution of saturated $\mathrm{Na}_{2} \mathrm{CO}_{3}(5 \mathrm{~mL})$ was added. The mixture was then allowed to stir at room temperature for $10 \mathrm{~min}$. The aqueous phase was extracted with dichloromethane $(3 \times 15 \mathrm{~mL})$ and the combined organic phases were dried over anhydrous $\mathrm{Na}_{2} \mathrm{SO}_{4}$. After removal of the solvent under reduced pressure, the residue was chromatographed on silica gel (petroleum ether 60-90 ${ }^{\circ} \mathrm{C}$ :ethyl acetate $\left.=7: 1\right)$ to afford the product $(\mathbf{2 8 a}$, $789 \mathrm{mg}, 95 \%$ yield) as a pale yellow syrup.

\subsection{1 $28 a$}

$[\alpha]_{\mathrm{D}}^{20}-278\left(\mathrm{c} 0.50, \mathrm{CHCl}_{3}\right) \cdot R_{\mathrm{f}} 0.45$ (petroleum ether:ethyl acetate $=7: 1)$. FTIR $(\mathrm{KBr}$, thin film $) \mathrm{cm}^{-1} 3435,2920$, 2352, 1703, 1615, 1361, 1047, 745, 602. ${ }^{1} \mathrm{H}-\mathrm{NMR}$ (400 MHz, $\left.\mathrm{CDCl}_{3}\right), \delta(\mathrm{ppm}) 7.32-7.19(2 \times 5 \mathrm{H}, m), 7.10$ $(2 \times 1 \mathrm{H}, t, J=7.2 \mathrm{~Hz}), 7.03(2 \times 1 \mathrm{H}, d, J=7.6 \mathrm{~Hz})$, $7.02(2 \times 1 \mathrm{H}, \quad t, \quad J=7.2 \mathrm{~Hz}), \quad 6.89(2 \times 1 \mathrm{H}, \quad t$, $J=7.6 \mathrm{~Hz}), 6.87(2 \times 1 \mathrm{H}, d, J=7.6 \mathrm{~Hz}), 6.69(2 \times 2 \mathrm{H}$, $d, \quad J=7.2 \mathrm{~Hz}), \quad 6.50(2 \times 1 \mathrm{H}, \quad t, \quad J=7.2 \mathrm{~Hz}), \quad 6.34$ $(2 \times 1 \mathrm{H}, d, J=7.6 \mathrm{~Hz}), 5.95(2 \times 1 \mathrm{H}, d d d, J=7.6$, $10.4,17.2 \mathrm{~Hz}), 5.19(2 \times 1 \mathrm{H}, d d, J=1.2,10.4 \mathrm{~Hz}), 4.86$ $(2 \times 1 \mathrm{H}, \quad d d, \quad J=1.2, \quad 17.2 \mathrm{~Hz}), 4.76(2 \times 1 \mathrm{H}, \quad d$, $J=15.6 \mathrm{~Hz}), \quad 4.38 \quad(2 \times 1 \mathrm{H}, \quad d, \quad J=15.6 \mathrm{~Hz}), \quad 3.80$ $(2 \times 1 \mathrm{H}, \quad d, \quad J=12.8 \mathrm{~Hz}), \quad 3.50-3.40 \quad(2 \times 2 \mathrm{H}, \quad m)$, 3.30-2.89 $(2 \times 2 \mathrm{H}, m) .{ }^{13} \mathrm{C}-\mathrm{NMR}\left(100 \mathrm{MHz}, \mathrm{CDCl}_{3}\right), \delta$ (ppm) $176.99,143.66,136.37,136.12,134.58,129.59$, $128.56,128.45,128.10,128.05,127.60,127.37,127.29$, $124.74,121.43,119.58,108.44,67.79,64.75,54.63,44.29$, 31.92. HRMS $\mathrm{m} / \mathrm{z}$ Found 831.3222, Calcd. for $\mathrm{C}_{52} \mathrm{H}_{49} \mathrm{Cl}_{2} \mathrm{~N}_{4} \mathrm{O}_{2}[\mathrm{M}+\mathrm{H}]^{+} 831.3233$.

\subsection{Synthesis of Compound 29}

To a solution of dichloroamine $\mathbf{2 8 a}$ (789 $\mathrm{mg}, 0.95 \mathrm{mmol})$ in anhydrous THF $(20 \mathrm{~mL})$ was added a powder of potassium tert-butoxide ( $t$-BuOK, $320 \mathrm{mg}, 2.85 \mathrm{mmol}, 3.0$ eq.). The resulting mixture was stirred at $0{ }^{\circ} \mathrm{C}$ for $1 \mathrm{~h}$. After which, the reaction mixture was treated with a solution of saturated $\mathrm{NH}_{4} \mathrm{Cl}(5 \mathrm{~mL})$. After removal of the solvents, the residue was diluted with water $(25 \mathrm{~mL})$ and extracted with dichloromethane $(3 \times 25 \mathrm{~mL})$, and the combined organic phases was dried over anhydrous sodium sulfate. After filtration and removal of the solvent under reduced pressure, the crude product was obtained $(670 \mathrm{mg}, 93 \%$ yield) as pale yellowish oil. The crude imine was dissolved in ethanol $(20 \mathrm{~mL})$. To this solution was added phenylhydrazine ( $0.26 \mathrm{~mL}, 2.64 \mathrm{mmol}, 3.0$ eq.). The resulting mixture was allowed to stir at room temperature for $3 \mathrm{~h}$. After removal of the solvent under reduced pressure, the residue was chromatographed on silica gel (dichloromethane: methanol: $\left.\mathrm{NH}_{3}-\mathrm{H}_{2} \mathrm{O}=400: 10: 1 \rightarrow 100: 10: 1\right)$ to afford the product $(\mathbf{2 9}, 489 \mathrm{mg}, 88 \%$ yield over two steps) as a pale yellow syrup.

$[\alpha]_{\mathrm{D}}^{20}-225\left(\mathrm{c} 0.28, \mathrm{CHCl}_{3}\right) \cdot R_{\mathrm{f}} 0.44\left(\mathrm{CH}_{2} \mathrm{Cl}_{2}: \mathrm{MeOH}=\right.$ 10:1). FTIR (KBr, thin film) $\mathrm{cm}^{-1} 3362,3055,1702,1607$, 1486, 1360, 1170, 997, 916, 751. ${ }^{1} H-N M R(400 \mathrm{MHz}$, $\left.\mathrm{CD}_{3} \mathrm{OD}\right), \delta(\mathrm{ppm}) 7.51(2 \times 2 \mathrm{H}, d, J=7.6 \mathrm{~Hz}), 7.43-7.33$ 
$(2 \times 3 \mathrm{H}, m), 7.05(2 \times 1 \mathrm{H}, d, J=8.0 \mathrm{~Hz}), 7.02(2 \times 1 \mathrm{H}$, $t, \quad J=7.6 \mathrm{~Hz}), \quad 6.77(2 \times 1 \mathrm{H}, \quad t, \quad J=7.6 \mathrm{~Hz}), \quad 6.61$ $(2 \times 1 \mathrm{H}, d, J=8.0 \mathrm{~Hz}), 5.50(2 \times 1 \mathrm{H}, d d d, J=7.2$, $10.4,17.2 \mathrm{~Hz}), 5.18(2 \times 1 \mathrm{H}, d, J=15.6 \mathrm{~Hz}), 4.64-4.53$ $(2 \times 3 \mathrm{H}, m), 3.31(2 \times 1 \mathrm{H}, d d, J=6.8,13.6 \mathrm{~Hz}), 2.93$ $(2 \times 1 \mathrm{H}, \quad d d, \quad J=6.4,13.2 \mathrm{~Hz}), 2.59(2 \times 1 \mathrm{H}, \quad d d$, $J=6.8,13.6 \mathrm{~Hz}) .{ }^{13} C-N M R\left(100 \mathrm{MHz}, \mathrm{CD}_{3} \mathrm{OD}\right), \delta(\mathrm{ppm})$ $179.10,144.37,142.14,137.21,129.64,129.46,128.76$, $128.60,125.45,122.76,114.37,109.83,56.14,52.99$, 45.11, 36.86. EI-MS m/z (\%) $583\left(\mathrm{M}^{+}+1,11 \%\right), 513$ (20), 496 (7), 444 (4), 292 (76), 291 (24), 275 (12), 261 (6), 236 (100), 235 (58), 223 (7), 206 (4), 158 (15), 130 (7), 91 (100). HRMS m/z Found 582.2991, Calcd. for $\mathrm{C}_{38} \mathrm{H}_{38} \mathrm{~N}_{4} \mathrm{O}_{2}$ $[\mathrm{M}]^{+}$582.2995.

\subsection{Synthesis of Compound $\mathbf{3 0}$}

Diamine 29 (291 mg, $0.5 \mathrm{mmol}$ ) in THF (20 mL) was degassed and purged with argon (three times). A solution of diisobutylaluminium hydride (DIBAL-H, 1.0 M in THF, $5 \mathrm{~mL}, 5 \mathrm{mmol}, 10.0$ eq.) was added at $0{ }^{\circ} \mathrm{C}$ and the resulting mixture was stirred at $0{ }^{\circ} \mathrm{C}$ for $1 \mathrm{~h}$, then at room temperature for $2 \mathrm{~h}$ and finally at $80^{\circ} \mathrm{C}$ (oil bath) under argon for $15 \mathrm{~h}$. After cooling to room temperature, a saturated aqueous solution of potassium sodium tartrate $(5 \mathrm{~mL})$ was added and the resulting mixture was stirred at room temperature for $2 \mathrm{~h}$. The mixture was diluted with water $(20 \mathrm{~mL})$ and extracted with ethyl acetate $(3 \times$ $20 \mathrm{~mL}$ ). The combined organic phases were dried over anhydrous sodium sulfate. After filtration and removal of the solvent, the residue was chromatographed on silica gel (petroleum ether $60-90{ }^{\circ} \mathrm{C}$ : ethyl acetate $=3: 1$ ) to afford the product $(30,143 \mathrm{mg}, 52 \%$ yield) as a yellow oil.

$[\alpha]_{\mathrm{D}}^{20}-210(\mathrm{c} 0.18, \mathrm{MeOH}) . R_{\mathrm{f}} 0.56$ (petroleum ether:ethyl acetate $=3: 1)$. FTIR $(\mathrm{KBr}$, thin film $) \mathrm{cm}^{-1} 3437$, 2919, 1601, 1491, 1349, 1088, 909, 738. ${ }^{1} H-N M R$ $\left(400 \mathrm{MHz}, \mathrm{CDCl}_{3}\right), \delta(\mathrm{ppm}) 7.30-7.12(2 \times 5 \mathrm{H}, \mathrm{m}), 7.03$ $(2 \times 1 \mathrm{H}, d, J=7.2 \mathrm{~Hz}), 6.92(2 \times 1 \mathrm{H}, t, J=7.2 \mathrm{~Hz})$, $6.48(2 \times 1 \mathrm{H}, \quad t, \quad J=7.2 \mathrm{~Hz}), \quad 6.20(2 \times 1 \mathrm{H}, \quad d$, $J=8.0 \mathrm{~Hz}), 5.64(2 \times 1 \mathrm{H}, d d d, J=6.8,10.4,17.2 \mathrm{~Hz})$, $5.00(2 \times 1 \mathrm{H}, \quad d, \quad J=17.2 \mathrm{~Hz}), 4.92(2 \times 1 \mathrm{H}, \quad d$, $J=10.4 \mathrm{~Hz}), 4.76(2 \times 1 \mathrm{H}, \quad s), 4.38(2 \times 1 \mathrm{H}, \quad d$, $J=16.0 \mathrm{~Hz}), 4.31(2 \times 1 \mathrm{H}, d, J=16.0 \mathrm{~Hz}), 3.40-3.30$ $(2 \times 1 \mathrm{H}, m), 2.10-1.97(2 \times 2 \mathrm{H}, m), 1.70(2 \times 1 \mathrm{H}$, brs $)$. ${ }^{13} \mathrm{C}-\mathrm{NMR}\left(100 \mathrm{MHz}, \mathrm{CDCl}_{3}\right), \delta$ (ppm) 152.27, 139.11, 139.00 , 131.43, 128.71, 128.60, 127.54, 127.20, 124.61, $116.56,115.58,105.21,85.91,62.82,59.74,49.15,44.84$. EI-MS m/z (\%) $550\left(\mathrm{M}^{+}, 10 \%\right), 308$ (3), 274 (100), 259 (3), 236 (3), 220 (19), 183 (18), 169 (5), 130 (7), 118 (6), 91 (62). HRMS m/z. Found 550.3096, Calcd. for $\mathrm{C}_{38} \mathrm{H}_{38} \mathrm{~N}_{4}$ $[\mathrm{M}]^{+} 550.3096$.

\subsection{Synthesis of Compound $\mathbf{3 1}$}

To a solution of liquid ammonia (freshly distilled and collected by Birch condenser, acetone-dry ice, 20-25 mL) at $-78^{\circ} \mathrm{C}$ was added sodium metal (ca. $60 \mathrm{mg}, 2.6 \mathrm{mmol}$, 10 eq.). A solution of diamine 30 (143 mg, $0.26 \mathrm{mmol})$ in anhydrous THF $(5 \mathrm{~mL})$ was added to this dark blue solution of liquid ammonia. After stirring at $-78^{\circ} \mathrm{C}$ for $15 \mathrm{~min}$, a powder of $\mathrm{NH}_{4} \mathrm{Cl}(214 \mathrm{mg}, 4 \mathrm{mmol})$ was added in one portion followed by saturated aqueous solution of $\mathrm{NH}_{4} \mathrm{Cl}(5 \mathrm{~mL})$. The resulting mixture was allowed to evaporate in fume hood. The residue was then diluted with water $(20 \mathrm{~mL})$ and extracted with dichloromethane $(3 \times$ $20 \mathrm{~mL}$ ). The organic phases were combined and dried over anhydrous sodium sulfate. After filtration, the solvent was removed under reduced pressure and the crude product was chromatographed on silica gel $\left(\mathrm{CH}_{2} \mathrm{Cl}_{2}: \mathrm{MeOH}: \mathrm{NH}_{3}-\right.$ $\left.\mathrm{H}_{2} \mathrm{O}=300: 10: 1\right)$ to afford the product $(\mathbf{3 1}, 92 \mathrm{mg}, 95 \%)$ as a yellow syrup.

$[\alpha]_{\mathrm{D}}^{20}-310(\mathrm{c} 0.30, \mathrm{MeOH}) . R_{\mathrm{f}} 0.48\left(\mathrm{CH}_{2} \mathrm{Cl}_{2}: \mathrm{MeOH}\right.$ : $\left.\mathrm{NH}_{3}-\mathrm{H}_{2} \mathrm{O}=30: 1: 0.1\right)$. FTIR $\left(\mathrm{KBr}\right.$, thin film) $\mathrm{cm}^{-1} 3404$, 2922, 2352, 1605, 1474, 1094, 928, 744. ${ }^{1} H-N M R$ $\left(400 \mathrm{MHz}, \quad \mathrm{CD}_{3} \mathrm{OD}\right), \quad \delta \quad(\mathrm{ppm}) 7.19(2 \times 1 \mathrm{H}, \quad d$, $J=7.2 \mathrm{~Hz}), 6.96(2 \times 1 \mathrm{H}, t, J=7.6 \mathrm{~Hz}), 6.62(2 \times 1 \mathrm{H}$, $t, \quad J=7.2 \mathrm{~Hz}), \quad 6.52(2 \times 1 \mathrm{H}, \quad d, \quad J=7.6 \mathrm{~Hz}), \quad 5.82$ $(2 \times 1 \mathrm{H}, d d d, J=7.2,10.4,17.2 \mathrm{~Hz}), 5.16(2 \times 1 \mathrm{H}, d$, $J=17.2 \mathrm{~Hz}), \quad 5.10(2 \times 1 \mathrm{H}, \quad d, \quad J=10.4 \mathrm{~Hz}), \quad 5.09$ $(2 \times 1 \mathrm{H}, s), 3.45-3.36(2 \times 1 \mathrm{H}, m), 2.29-2.17(2 \times 2 \mathrm{H}$, m). ${ }^{13} C-N M R \quad\left(100 \mathrm{MHz}, \mathrm{CD}_{3} \mathrm{OD}\right), \delta$ (ppm) 152.62, 138.09 , 131.63, 129.70, 125.36, 119.32, 117.74, 109.82, 81.39, 65.04, 61.28, 43.61. EI-MS m/z (\%) $370\left(\mathrm{M}^{+}\right.$, $22 \%), 314$ (4), 260 (5), 245 (11), 232 (4), 219 (3), 184 (100), 183 (22), 169 (13), 158 (6), 131 (27), 130 (30), 117 (4), 103 (4), 91 (3). HRMS m/z Found 370.2155, Calcd. for $\mathrm{C}_{24} \mathrm{H}_{26} \mathrm{~N}_{4}[\mathrm{M}]^{+} 370.2157$.

\subsection{Synthesis of Compound $\mathbf{3 3}$}

To a solution of amine 31 (74 $\mathrm{mg}, 0.2 \mathrm{mmol}$ ) and FMOC$(S)$-MePhe-OH (200 mg, $0.5 \mathrm{mmol}, 2.5$ eq.) in anhydrous DMF ( $3 \mathrm{~mL})$ was added $\mathrm{Et}_{3} \mathrm{~N}(0.14 \mathrm{~mL}, 1 \mathrm{mmol}, 5.0$ eq.) and $O$-(7-azabenzotriazol-1-yl)- $N, N, N^{\prime}, N^{\prime}$-tetramethyluronium hexafluoro-phosphate (HATU, $190 \mathrm{mg}, 0.5 \mathrm{mmol}$, 2.5 eq.) at $0{ }^{\circ} \mathrm{C}$. The resulting mixture was then stirred at room temperature for $12 \mathrm{~h}$. The mixture was then treated with an aqueous solution of $\mathrm{LiCl}(5 \%$, ca. $10 \mathrm{~mL})$. After stirring for $5 \mathrm{~min}$, the mixture was diluted with water $(10 \mathrm{~mL})$ and extracted with ethyl acetate $(3 \times 20 \mathrm{~mL})$. The combined organic phases were washed with $5 \%$ aqueous solution of $\mathrm{LiCl}(5 \mathrm{~mL})$ and dried over anhydrous sodium sulfate. After filtration, the solvent was removed under 
reduced pressure to afford the product (32, $198 \mathrm{mg}, 87 \%$ ) which was used directly in the next step without further purification. To a solution of olefin 32 (114 $\mathrm{mg}, 0.1 \mathrm{mmol})$ in THF $(3 \mathrm{~mL})$ and $\mathrm{H}_{2} \mathrm{O}(1 \mathrm{~mL})$ was added 4-methylmorpholine- $N$-oxide ( $47 \mathrm{mg}, 0.4 \mathrm{mmol}, 4.0$ eq.) and $\mathrm{OsO}_{4}$ (20 mg/mL in water, $0.13 \mathrm{~mL}, 0.01 \mathrm{mmol}, 0.1$ eq.). The resulting mixture was then stirred at room temperature for $20 \mathrm{~h}$ under nitrogen. After which, $\mathrm{NaIO}_{4}$ (86 mg, $0.4 \mathrm{mmol}, 4.0$ eq.) was added and the mixture was allowed to stir at room temperature for $3 \mathrm{~h}$. The resulting mixture was then diluted with water $(10 \mathrm{~mL})$ and extracted with ethyl acetate $(3 \times 15 \mathrm{~mL})$. The organic phases were combined and dried over anhydrous sodium sulfate. After filtration and removal of the solvent, the residue was chromatographed on silica gel (petroleum ether 60-90 ${ }^{\circ} \mathrm{C}$ :ethyl acetate $=2: 1$ ) to afford the dialdehyde $(\mathbf{3 3}, 106 \mathrm{mg}, 93 \%)$ as white plates.

m.p. $155-157{ }^{\circ} \mathrm{C},[\alpha]_{\mathrm{D}}^{20}-318\left(\mathrm{c} 0.35, \mathrm{CHCl}_{3}\right) \cdot R_{\mathrm{f}} 0.41$ (petroleum ether:ethyl acetate $=3: 1)$. FTIR $(\mathrm{KBr}$, thin film) $\mathrm{cm}^{-1} 3425,2920,1730,1666,1450,1321,1147$, 1071, 744. ${ }^{1} \mathrm{H}-\mathrm{NMR}\left(400 \mathrm{MHz}, \mathrm{CDCl}_{3}\right), \delta$ (ppm) 8.95 $(2 \times 1 \mathrm{H}, d, J=4.4 \mathrm{~Hz}), 7.78(2 \times 2 \mathrm{H}, d, J=7.6 \mathrm{~Hz})$, $7.58-7.00(2 \times 12 \mathrm{H}, m), 6.94(2 \times 1 \mathrm{H}, t, J=7.6 \mathrm{~Hz})$, $6.63(2 \times 1 \mathrm{H}, \quad t, \quad J=7.6 \mathrm{~Hz}), \quad 6.32(2 \times 1 \mathrm{H}, \quad d$, $J=7.6 \mathrm{~Hz}), 5.12(2 \times 1 \mathrm{H}, t, J=8.0 \mathrm{~Hz}), 4.92(2 \times 1 \mathrm{H}$, $s), 4.48(2 \times 1 \mathrm{H}, d d, J=7.2,10.4 \mathrm{~Hz}), 4.36(2 \times 1 \mathrm{H}, d d$, $J=7.2, \quad 10.4 \mathrm{~Hz}), \quad 4.25 \quad(2 \times 1 \mathrm{H}, \quad t, \quad J=6.8 \mathrm{~Hz})$, 3.81-3.74 $(2 \times 1 \mathrm{H}, m), \quad 3.12(2 \times 3 \mathrm{H}, s), \quad 2.97-2.85$ $(2 \times 2 \mathrm{H}, m), 2.08-2.00(2 \times 1 \mathrm{H}, m), 1.92-1.82(2 \times 1 \mathrm{H}$, $m) .{ }^{13} C-N M R\left(100 \mathrm{MHz}, \mathrm{CDCl}_{3}\right), \delta$ (ppm) 195.77, 170.75, $157.86,149.38,143.84,143.66,141.47,135.72,130.06$, $129.08,128.99,128.03,127.99,127.71,127.29,126.22$, $125.17,125.08,123.80,120.24,118.77,109.18,79.14$, $68.60,64.42,62.14,56.28,47.23,36.83,32.84,30.40$. HRMS m/z Found 1141.4830, Calcd. for $\mathrm{C}_{72} \mathrm{H}_{65} \mathrm{~N}_{6} \mathrm{O}_{8}$ $(\mathrm{M}+\mathrm{H})^{+}$1141.4864; Found 1163.4662, Calcd. for $\mathrm{C}_{72} \mathrm{H}_{64} \mathrm{~N}_{6} \mathrm{NaO}_{8}[\mathrm{M}+\mathrm{Na}]^{+} 1163.4683$.

\subsection{Synthesis of (-)-Ditryptophenaline}

To a solution of dialdehyde 33 (106 $\mathrm{mg}, 0.093 \mathrm{mmol}$ ) in THF $(2 \mathrm{~mL})$, water $(2 \mathrm{~mL})$ and $t$ - $\mathrm{BuOH}(0.5 \mathrm{~mL})$ was added 2-methyl-2-butene $(1 \mathrm{~mL}), \mathrm{NaClO}_{2}$ (80\% purity, $105 \mathrm{mg}, 0.93 \mathrm{mmol}, 10$ eq.) and $\mathrm{KH}_{2} \mathrm{PO}_{4}(253 \mathrm{mg}$, $1.86 \mathrm{mmol}, 20$ eq.). The resulting mixture was then stirred at room temperature for $15 \mathrm{~h}$. After which, the mixture was diluted with a saturated aqueous solution of $\mathrm{NH}_{4} \mathrm{Cl}$ $(40 \mathrm{~mL})$ and extracted with ethyl acetate $(3 \times 20 \mathrm{~mL})$. The combined organic phases were washed with brine $(10 \mathrm{~mL})$ and dried over anhydrous sodium sulfate. After filtration and removal of the solvent, the crude product was obtained (101 mg, $93 \%)$ and used in next step without further purification. The diacid $(101 \mathrm{mg})$ was dissolved in THF $(3 \mathrm{~mL})$ and treated with piperidine $(0.5 \mathrm{~mL})$ at room temperature for $4 \mathrm{~h}$. After removal of solvent and excess piperidine, the crude yellowish oil $(60 \mathrm{mg})$ was obtained and used in next step without further purification. To a solution of aminoacid $(60 \mathrm{mg}$, $\sim 0.082 \mathrm{mmol})$ in dichloromethane $(4 \mathrm{~mL})$ was added DCC (169 mg, $0.82 \mathrm{mmol}, 10 \mathrm{eq}$.). The resulting mixture was stirred at $40{ }^{\circ} \mathrm{C}$ for $15 \mathrm{~h}$. After removal of the solvent, the residue was chromatographed on silica gel (petroleum ether $60-90{ }^{\circ} \mathrm{C}$ :ethyl acetate $=3: 1 \rightarrow 1: 5$ ) to afford the product $(\mathbf{5}, 50 \mathrm{mg}, 78 \%$ over three steps) as white plates.

m.p. $201-202{ }^{\circ} \mathrm{C},[\alpha]_{\mathrm{D}}^{20}-292\left(\mathrm{c} 0.40, \mathrm{CH}_{2} \mathrm{Cl}_{2}\right) \cdot R_{\mathrm{f}} 0.41$ (petroleum ether:ethyl acetate $=1: 5)$. FTIR $(\mathrm{KBr}$, thin film) $\mathrm{cm}^{-1} 3426,2921,1651,1452,1075,749 .{ }^{1} H-N M R$ $\left(400 \mathrm{MHz}, \mathrm{CDCl}_{3}\right), \delta(\mathrm{ppm}) 7.55(2 \times 2 \mathrm{H}, t, J=7.6 \mathrm{~Hz})$, $7.50 \quad(2 \times 1 \mathrm{H}, \quad t, \quad J=7.2 \mathrm{~Hz}), \quad 7.13 \quad(2 \times 2 \mathrm{H}, \quad d$, $J=7.2 \mathrm{~Hz}), 7.06(2 \times 1 \mathrm{H}, d t, J=0.8,7.6 \mathrm{~Hz}), 6.96$ $(2 \times 1 \mathrm{H}, d, J=7.6 \mathrm{~Hz}), 6.69(2 \times 1 \mathrm{H}, t, J=7.2 \mathrm{~Hz})$, $6.54(2 \times 1 \mathrm{H}, d, J=7.6 \mathrm{~Hz}), 4.80(2 \times 1 \mathrm{H}, s), 4.68$ $(2 \times 1 \mathrm{H}, s), 4.27-4.22(2 \times 1 \mathrm{H}, m), 3.65(2 \times 1 \mathrm{H}, d d$, $J=4.8,12.0 \mathrm{~Hz}), 3.52(2 \times 1 \mathrm{H}, d d, J=3.0,14.4 \mathrm{~Hz})$, $3.24(2 \times 1 \mathrm{H}, d d, J=4.4,14.4 \mathrm{~Hz}), 3.02(2 \times 3 \mathrm{H}, s)$, $2.01(2 \times 1 \mathrm{H}, d d, J=4.8,12.4 \mathrm{~Hz}), 1.56(2 \times 1 \mathrm{H}, t$, $J=12.4 \mathrm{~Hz}) .{ }^{13} \mathrm{C}-\mathrm{NMR}\left(100 \mathrm{MHz}, \mathrm{CDCl}_{3}\right), \delta(\mathrm{ppm})$ $165.55,164.10,150.30,134.64,129.78,129.52,129.46$, $128.07,126.62,125.89,119.08,109.78,63.27,59.09$, 58.70, 36.34, 36.14, 32.69. HRMS $\mathrm{m} / \mathrm{z}$. Found 693.3178, Calcd. for $\mathrm{C}_{42} \mathrm{H}_{41} \mathrm{~N}_{6} \mathrm{O}_{4}[\mathrm{M}+\mathrm{H}]^{+} 693.3189$.

Acknowledgments This work was supported by Grants from Natural Science Foundation of China (20925205 and 21332007), the Program for Changjiang Scholars and Innovative Research Team in University (IRT13095), Yunnan Provincial Science and Technology Department (2010GA014) and the Program for Yunling Scholars. Dr. Xiaonian $\mathrm{Li}$ of the Kunming Institute of Botany is gratefully acknowledged for X-ray crystallographic analysis of compound $\mathbf{2 3}$. CCDC 1409209 (23).

Open Access This article is distributed under the terms of the Creative Commons Attribution 4.0 International License (http:// creativecommons.org/licenses/by/4.0/), which permits unrestricted use, distribution, and reproduction in any medium, provided you give appropriate credit to the original author(s) and the source, provide a link to the Creative Commons license, and indicate if changes were made.

\section{References}

1. G.A. Cordell, J.E. Saxton, in The Alkaloids: Chemistry and Physiology, vol. 20, ed. by R.H.F. Manske, R.G.A. Rodrigo (Academic, New York, 1981), p. 3

2. T. Hino, M. Nakagawa, in The Alkaloids: Chemistry and Pharmacology, vol. 34, ed. by A. Brossi (Academic, San Diego, 1988), p. 1 
3. U. Anthoni, C. Christophersen, P.H. Nielsen, in Alkaloids: Chemical and Biological Perspectives, vol. 13, ed. by S.W. Pelletier (Pergamon, Amsterdam, 1999), p. 163

4. T.-S. Kam, Y.-M. Choo, in The Alkaloids: Chemistry and Biology, vol. 63, ed. by G.A. Cordell (Academic, New York, 2006), p. 181

5. L.A. Sorbera, J. Castaner, Drug Future 28, 18 (2003)

6. P.E. Santos, P.S. Almeida, A.M. Lobo, S. Prabhakar, Heterocycles 55, 1029 (2001)

7. A. Al-Jafari, M.A. Kamal, N.H. Greig, A.S. Alhomida, E.R. Perry, Biochem. Biophys. Res. Commun. 248, 180 (1998)

8. P. Ruiz-Sanchis, S.A. Savina, F. Albericio, M. Álvarez, Chem. Eur. J. 17, 1388 and references cited therein (2011)

9. J.F. Austin, S.G. Kim, C.J. Sinz, W.J. Xiao, D.W.C. MacMillan, PNAS 101, 5482 (2004)

10. E.A. Peterson, L.E. Overman, PNAS 101, 11943 (2004)

11. C.J. Douglas, L.E. Overman, PNAS 101, 5363 (2004)

12. J. Kim, M. Movassaghi, Chem. Soc. Rev. 38, 3035 (2009)

13. D. Crich, A. Banerjee, Acc. Chem. Res. 40, 151 (2007)

14. A. Steven, L.E. Overman, Angew. Chem. Int. Ed. 46, 5488 (2007)

15. M.A. Schmidt, M. Movassaghi, Synlett. 19, 313 (2008)

16. L.M. Repka, S.E. Reisman, J. Org. Chem. 78, 12314 (2013)

17. J.T. Link, L.E. Overman, J. Am. Chem. Soc. 118, 8166 (1996)

18. T. Matsuura, L.E. Overman, D.J. Poon, J. Am. Chem. Soc. 120, 6500 (1998)

19. L.E. Overman, D.V. Paone, B.A. Stearns, J. Am. Chem. Soc. 121, 7702 (1999)

20. L.E. Overman, J.F. Larrow, B.A. Stearns, J.M. Vance, Angew. Chem. Int. Ed. 39, 213 (2000)

21. L.E. Overman, D.V. Paone, J. Am. Chem. Soc. 123, 9465 (2001)

22. A.B. Dounay, K. Hatanaka, J.J. Kodanko, M. Oestreich, L.E. Overman, L.A. Pfeifer, M.M. Weiss, J. Am. Chem. Soc. 125, 6261 (2003)

23. L.E. Overman, E.A. Peterson, Angew. Chem. Int. Ed. 42, 2525 (2003)

24. J.J. Kodanko, L.E. Overman, Angew. Chem. Int. Ed. 42, 2528 (2003)

25. L.E. Overman, E.A. Peterson, Tetrahedron 59, 6905 (2003)

26. A. Huang, J.J. Kodanko, L.E. Overman, J. Am. Chem. Soc. 126, 14043 (2004)

27. J.J. Kodanko, S. Hiebert, E.A. Peterson, L. Sung, L.E. Overman, V.M. Linck, G.C. Goerck, T.A. Amador, M.B. Leal, E. Elisabetsky, J. Org. Chem. 72, 7909 (2007)

28. M. Movassaghi, M.A. Schmidt, Angew. Chem. Int. Ed. 46, 3725 (2007)

29. M. Movassaghi, M.A. Schmidt, J.A. Ashenhurst, Angew. Chem. Int. Ed. 47, 1485 (2008)

30. J. Kim, J.A. Ashenhurst, M. Movassaghi, Science 324, 238 (2009)

31. J. Kim, M. Movassaghi, J. Am. Chem. Soc. 132, 14376 (2010)

32. C. Pérez-Balado, Á.R. de Lera, Org. Lett. 10, 3701 (2008)

33. C. Pérez-Balado, P. Rodríguez-Graña, Á.R. de Lera, Chem. Eur. J. 15, 9928 (2009)

34. E. Iwasa, Y. Hamashima, S. Fujishiro, E. Higuchi, A. Ito, M. Yoshida, M. Sodeoka, J. Am. Chem. Soc. 132, 4078 (2010)

35. W. Xie, G. Jiang, H. Liu, J. Hu, X. Pan, H. Zhang, X. Wan, Y. Lai, D. Ma, Angew. Chem. Int. Ed. 52, 12924 (2013)

36. Y. Peng, L. Luo, C. Yan, J. Zhang, Y. Wang, J. Org. Chem. 78, 10960 (2013)

37. M. Wada, T. Murata, H. Oikawa, H. Oguri, Org. Biomol. Chem. 12, 298 (2014)

38. T. Hino, S.-I. Yamada, Tetrahedron Lett. 4, 1757 (1963)

39. A.I. Scott, F. McCapra, E.S. Hall, J. Am. Chem. Soc. 86, 302 (1964)
40. E.S. Hall, F. McCapra, A.I. Scott, Tetrahedron 23, 4131 (1967)

41. M. Nakagawa, H. Sugumi, S. Kodato, T. Hino, Tetrahedron Lett. 22, 5323 (1981)

42. H. Ishikawa, H. Takayama, N. Aimi, Tetrahedron Lett. 43, 5637 (2002)

43. L. Verotta, F. Orsini, M. Sbacchi, M.A. Scheidler, T.A. Amador, E. Elisabetsky, Bioorg. Med. Chem. 10, 2133 (2002)

44. H. Takayama, Y. Matsuda, K. Masubuchi, A. Ishida, M. Kitajima, N. Aimi, Tetrahedron 60, 893 (2004)

45. Y. Matsuda, M. Kitajima, H. Takayama, Heterocycles 65, 1031 (2005)

46. R.H. Snell, R.L. Woodward, M.C. Willis, Angew. Chem. Int. Ed. 50, $9116(2011)$

47. R.H. Snell, M.J. Durbin, R.L. Woodward, M.C. Willis, Chem. Eur. J. 18, 16754 (2012)

48. Y.-X. Li, H.-X. Wang, S. Ali, X.-F. Xia, Y.-M. Liang, Chem. Commun. 48, 2343 (2012)

49. S. Tadano, Y. Mukaeda, H. Ishikawa, Angew. Chem. Int. Ed. 52, 7990 (2013)

50. D. Sun, C. Xing, X. Wang, Z. Su, C. Li, Org. Chem. Front. 1, 956 (2014)

51. K. Liang, X. Deng, X. Tong, D. Li, M. Ding, A. Zhou, C. Xia, Org. Lett. 17, 206 (2015)

52. M. Ding, K. Liang, R. Pan, H. Zhang, C. Xia, J. Org. Chem. 80, 10309 (2015)

53. K.M. Depew, S.P. Marsden, D. Zatorska, A. Zatorski, W.G. Bornmann, S.J. Danishefsky, J. Am. Chem. Soc. 121, 11953 (1999)

54. M. Somei, N. Oshikiri, M. Hasegawa, F. Yamada, Heterocycles 51, 1237 (1999)

55. C. Menozzi, P.I. Dalko, J. Cossy, Chem. Commun. 44, 4638 (2006)

56. M. Movassaghi, O.K. Ahmad, S.P. Lathrop, J. Am. Chem. Soc. 133, 13002 (2011)

57. S.P. Lathrop, M. Movassaghi, Chem. Sci. 5, 333 (2014)

58. M. Tayu, K. Higuchi, T. Ishizaki, T. Kawasaki, Org. Lett. 16, 3613 (2014)

59. T. Araki, Y. Manabe, K. Fujioka, H. Yokoe, M. Kanematsu, M. Yoshida, K. Shishido, Tetrahedron Lett. 54, 1012 (2013)

60. Y. Zhao, Y. Zhou, L. Liang, X. Yang, F. Du, L. Li, H. Zhang, Org. Lett. 11, 555 (2009)

61. Y. Zhou, Y. Zhao, X. Dai, J. Liu, L. Li, H. Zhang, Org. Biomol. Chem. 9, 4091 (2011)

62. Y. Zhou, Y. Xi, J. Zhao, X. Sheng, S. Zhang, H. Zhang, Org. Lett. 14, 3116 (2012)

63. H. Zhang, Synlett 25, 1953 (2014)

64. X. Shen, Y. Zhou, Y. Xi, J. Zhao, H. Zhang, Chem. Commun. 51, $14873(2015)$

65. S. Tadano, H. Ishikawa, Synlett 25, 157 (2014)

66. J.B. Hendrickson, R. Göschke, R. Rees, Tetrahedron 20, 565 (1964)

67. A. Inada, Y. Morita, Heterocycles 19, 2139 (1982)

68. C.-L. Fang, S. Horne, N. Taylor, R. Rodrigo, J. Am. Chem. Soc. 116, 9480 (1994)

69. H.J. Lee, S. Lee, J.W. Lim, J.N. Kim, Bull. Korean Chem. Soc. 34, 2446 (2013)

70. S. Ghosh, S. Chaudhuri, A. Bisai, Org. Lett. 17, 1373 (2015)

71. C. Guo, J. Song, J.-Z. Huang, P.-H. Chen, S.-W. Luo, L.-Z. Gong, Angew. Chem. Int. Ed. 51, 1046 (2012)

72. H. Mitsunuma, M. Shibasaki, M. Kanai, S. Matsunaga, Angew. Chem. Int. Ed. 51, 5217 (2012)

73. R. Liu, J. Zhang, Org. Lett. 15, 2266 (2013) 
74. B.M. Trost, M. Osipov, Angew. Chem. Int. Ed. 52, 9176 (2013)

75. S. Ghosh, S. Bhunia, B.N. Kakde, S. De, A. Bisai, Chem. Commun. 50, 2434 (2014)

76. G.B. Jones, B.J. Chapman, Synthesis 5, 475 (1995)

77. J.G. Ruano, A. Parra, V. Marcos, C. del Pozo, S. Catalán, S. Monteagudo, S. Fustero, A. Poveda, J. Am. Chem. Soc. 131, 9432 (2009)
78. D.A. Cogan, G. Liu, J. Ellman, Tetrahedron 55, 8883 (1999)

79. R.A. Olofson, J.T. Martz, J.-P. Senet, M. Piteau, T. Malfroot, J. Org. Chem. 49, 2081 (1984)

80. T. Vidal, E. Magnier, Y. Langlois, Tetrahedron 54, 5959 (1998)

81. M.-J. Chen, Y.-M. Tsai, Tetrahedron 67, 1564 (2011) 